\title{
Optimal inference in a class of regression models*
}

\author{
Timothy B. Armstrong ${ }^{\dagger} \quad$ Michal Kolesár ${ }^{\ddagger}$ \\ Yale University Princeton University
}

November 23, 2017

\begin{abstract}
We consider the problem of constructing confidence intervals (CIs) for a linear functional of a regression function, such as its value at a point, the regression discontinuity parameter, or a regression coefficient in a linear or partly linear regression. Our main assumption is that the regression function is known to lie in a convex function class, which covers most smoothness and/or shape assumptions used in econometrics. We derive finite-sample optimal CIs and sharp efficiency bounds under normal errors with known variance. We show that these results translate to uniform (over the function class) asymptotic results when the error distribution is not known. When the function class is centrosymmetric, these efficiency bounds imply that minimax CIs are close to efficient at smooth regression functions. This implies, in particular, that it is impossible to form CIs that are substantively tighter using data-dependent tuning parameters, and maintain coverage over the whole function class. We specialize our results to inference on the regression discontinuity parameter, and illustrate them in simulations and an empirical application.
\end{abstract}

${ }^{*}$ We thank Don Andrews, Isaiah Andrews, Matias Cattaneo, Gary Chamberlain, Denis Chetverikov, Yuichi Kitamura, Soonwoo Kwon, Ulrich Müller and Azeem Shaikh for useful discussions. We thank the editor, three anonymous referees, and numerous seminar and conference participants for helpful comments and suggestions. All remaining errors are our own. The research of the first author was supported by National Science Foundation Grant SES-1628939. The research of the second author was supported by National Science Foundation Grant SES-1628878.

${ }^{\dagger}$ email: timothy.armstrong@yale.edu

$\ddagger$ email: mkolesar@princeton.edu 


\section{Introduction}

In this paper, we study the problem of constructing confidence intervals (CIs) for a linear functional $L f$ of a regression function $f$ in a broad class of regression models with fixed regressors, in which $f$ is known to belong to some convex function class $\mathcal{F}$. The linear functional may correspond to the regression discontinuity parameter, an average treatment effect under unconfoundedness, or a regression coefficient in a linear or partly linear regression. The class $\mathcal{F}$ may contain smoothness restrictions (e.g. bounds on derivatives, or assuming $f$ is linear as in a linear regression), and/or shape restrictions (e.g. monotonicity, or sign restrictions on regression coefficients in a linear regression). Often in applications, the function class will be indexed by a smoothness parameter $C$, such as when $\mathcal{F}=\mathcal{F}_{\text {Lip }}(C)$, the class of Lipschitz continuous functions with Lipschitz constant $C$.

Our main contribution is to derive finite-sample optimal CIs and sharp efficiency bounds that have implications for data-driven model and bandwidth selection in both parametric and nonparametric settings. To derive these results, we assume that the regression errors are normal, with known variance. When the error distribution is unknown, we obtain analogous uniform asymptotic results under high-level regularity conditions. We derive sufficient lowlevel conditions in an application to regression discontinuity.

First, we characterize one-sided CIs that minimize the maximum $\beta$ quantile of excess length over a convex class $\mathcal{G}$ for a given quantile $\beta$. The lower limit $\hat{c}$ of the optimal CI $[\hat{c}, \infty)$ has a simple form: take an estimator $\hat{L}$ that trades off bias and variance in a certain optimal sense and is linear in the outcome vector, and subtract (1) the standard deviation of $\hat{L}$ times the usual critical value based on a normal distribution and (2) a bias correction to ensure coverage. This bias correction, in contrast to bias corrections often used in practice, is based on the maximum bias of $\hat{L}$ over $\mathcal{F}$, and is therefore non-random.

When $\mathcal{G}=\mathcal{F}$, this procedure yields minimax one-sided CIs. Setting $\mathcal{G} \subset \mathcal{F}$ to a class of smoother functions is equivalent to "directing power" at these smoother functions while maintaining coverage over $\mathcal{F}$, and gives a sharp bound on the scope for adaptation for one-

sided CIs. We show that when $\mathcal{F}$ is centrosymmetric (i.e. $f \in \mathcal{F}$ implies $-f \in \mathcal{F}$ ), the scope for adaptation is severely limited: when $\mathcal{G}$ is a class of functions that are, in a certain formal sense, "sufficiently smooth" relative to $\mathcal{F}$, CIs that are minimax for $\beta$ quantile of excess length also optimize excess length over $\mathcal{G}$, but at a different quantile. Furthermore, they are also highly efficient at such smooth functions for the same quantile. For instance, a CI for the conditional mean at a point that is minimax over the Lipschitz class $\mathcal{F}_{\text {Lip }}(C)$ is asymptotically $95.2 \%$ efficient at constant functions relative to a CI that directs all power 
at constant functions. For function classes that bound a derivative of higher order, the efficiency is even higher.

Second, we derive a confidence set that minimizes its expected length at a single function g. We compare its performance to the optimal fixed-length CI of Donoho (1994) (i.e. CI of the form $\hat{L} \pm \chi$, where $\hat{L}$ is an affine estimator, and $\chi$, which doesn't depend on the outcome vector and is therefore non-random, is chosen to ensure coverage). Similarly, to the onesided case, we find that, when $\mathcal{F}$ is centrosymmetric, the optimal fixed-length CIs are highly efficient at functions that are smooth relative to $\mathcal{F}$. For instance, the optimal fixed-length CI for a conditional mean at a point when $f \in \mathcal{F}_{\text {Lip }}(C)$ is asymptotically $95.6 \%$ efficient at any constant function $g$ relative to a confidence set that optimizes its expected length at $g$.

An important practical implication of these results is that explicit a priori specification of the smoothness constant $C$ cannot be avoided: procedures that try to determine the smoothness of $f$ from the data (and thus implicitly estimate $C$ from the data), including data-driven bandwidth or variable selectors, must either fail to substantively improve upon the minimax CIs or fixed-length CIs (that effectively assume the worst case smoothness), or else fail to maintain coverage over the whole parameter space. We illustrate this point through a Monte Carlo study in a regression discontinuity (RD) setting, in which we show that popular data-driven bandwidth selectors lead to substantial undercoverage, even when combined with bias correction or undersmoothing (see Supplemental Appendix C.2). To avoid having to specify $C$, one has to strengthen the assumptions on $f$. For instance, one can impose shape restrictions that break the centrosymmetry, as in Cai et al. (2013) or Armstrong (2015), or self-similarity assumptions that break the convexity, as in Giné and Nickl (2010) or Chernozhukov et al. (2014). Alternatively, one can weaken the coverage requirement in the definition of a CI, by, say, only requiring average coverage as in Cai et al. (2014) or Hall and Horowitz (2013).

We apply these results to the problem of inference in RD. We show, in the context of an empirical application from Lee (2008), that the fixed-length and minimax CIs are informative and simple to construct, and we give a detailed guide to implementing them in practice. We also consider CIs based on local linear estimators, which have been popular in RD due to their high minimax asymptotic MSE efficiency, shown in Cheng et al. (1997). Using the same function classes as in Cheng et al. (1997), we show that in the Lee application, when a triangular kernel is used, such CIs are highly efficient relative to the optimal CIs discussed above.

Our finite-sample approach allows us to use the same framework and methods to cover 
problems that are often seen as outside of the scope of nonparametric methods. For instance, the same CIs can be used in RD whether the running variable is discrete or continuous; one does not need a different modeling approach, such as that of Lee and Card (2008). Similarly, we do not need to distinguish between "parametric" or "nonparametric" constraints on $f$; our results apply to inference in a linear regression model that efficiently use a priori bounds and sign restrictions on the regression coefficients. Here our efficiency bounds imply that the scope for efficiency improvements from CIs formed after model selection (Andrews and Guggenberger, 2009; McCloskey, 2017) is severely limited unless asymmetric or non-convex restrictions are imposed, and they also limit the scope for improvement under certain nonconvex restrictions such as the sparsity assumptions used in Belloni et al. (2014). We discuss these issues in an earlier version of this paper (Armstrong and Kolesár, 2016a).

Our results and setup build on a large statistics literature on optimal estimation and inference in the nonparametric regression model. This literature has mostly been concerned with estimation (e.g., Stone (1980), Ibragimov and Khas'minskii (1985), Fan (1993), Donoho (1994), Cheng et al. (1997)); the literature on inference has mostly been focused on bounding rates of convergence. The results most closely related to ours are those in Low (1997), Cai and Low (2004a) and Cai et al. (2013), who derive lower bounds on the expected length of a two-sided CI over a convex class $\mathcal{G}$ subject to coverage over a convex class $\mathcal{F}$. These results imply that, when $\mathcal{F}$ is constrained only by bounds on a derivative, one cannot improve the rate at which a two-sided CI shrinks by "directing power" at smooth functions. We contribute to this literature by (1) deriving a sharp lower bound for one-sided CIs, and for two-sided CIs when $\mathcal{G}$ is a singleton, (2) showing that the negative results for "directing power" at smooth functions generalize to the case when $\mathcal{F}$ is centrosymmetric, and deriving the sharp bound on the scope for improvement, (3) deriving feasible CIs under unknown error distribution and showing their asymptotic validity and efficiency, including in non-regular settings; and (4) computing the bounds and CIs in an application to RD.

The remainder of this paper is organized as follows. Section 2 illustrates our results in an application to RD, and gives a detailed guide to implementing our CIs. Section 3 derives the main results under a general setup. Section 4 considers an empirical application. Proofs, long derivations, and additional results are collected in appendices. Appendix A contains proofs for the main results in Section 3. Appendix B discusses extensions to incorporate covariates in the RD application. Supplemental Appendix C compares our CIs to other approaches, and includes a Monte Carlo study. Additional details for constructing CIs studied in Section 3 are in Supplemental Appendix D. Supplemental Appendix E contains 
additional details for the RD application. Asymptotic results are collected in Supplemental Supplemental Appendices F, G and H.

\section{Application to regression discontinuity}

In this section, we explain our results in the context of an application to sharp regression discontinuity (RD). Section 2.1 illustrates the theoretical results, while Section 2.2 gives step-by-step instructions for implementing our confidence intervals (CIs) in practice.

We observe $\left\{y_{i}, x_{i}\right\}_{i=1}^{n}$, where the running variable $x_{i}$ is deterministic, and

$$
y_{i}=f\left(x_{i}\right)+u_{i}, \quad u_{i} \sim \mathcal{N}\left(0, \sigma^{2}\left(x_{i}\right)\right) \text { independent across } i,
$$

with $\sigma^{2}(x)$ known. ${ }^{1}$ The running variable determines participation in a binary treatment: units above a given cutoff, which we normalize to 0 , are treated; units with $x_{i}<0$ are controls. Let $f_{+}(x)=f(x) 1(x \geq 0)$ and $f_{-}(x)=f(x) 1(x<0)$ denote the part of the regression function $f$ above and below the cutoff, so that $f=f_{+}+f_{-}$. The parameter of interest is the jump of the regression function at zero, and we denote it by $L f=f_{+}(0)-f_{-}(0)$, where $f_{-}(0)=\lim _{x \uparrow 0} f_{-}(x)$. If the regression functions of potential outcomes are continuous at zero, then $L f$ measures the average treatment effect for units with $x_{i}=0$.

We assume that $f$ lies in the class of functions $\mathcal{F}_{R D T, p}(C)$,

$$
\mathcal{F}_{R D T, p}(C)=\left\{f_{+}+f_{-}: f_{+} \in \mathcal{F}_{T, p}\left(C ; \mathbb{R}_{+}\right), f_{-} \in \mathcal{F}_{T, p}\left(C ; \mathbb{R}_{-}\right)\right\}
$$

where $\mathcal{F}_{T, p}(C ; \mathcal{X})$ consists of functions $f$ such that the approximation error from a $(p-1)$ thorder Taylor expansion of $f(x)$ about 0 is bounded by $C|x|^{p}$, uniformly over $\mathcal{X}$,

$$
\mathcal{F}_{T, p}(C ; \mathcal{X})=\left\{f:\left|f(x)-\sum_{j=0}^{p-1} f^{(j)}(0) x^{j} / j !\right| \leq C|x|^{p} \text { all } x \in \mathcal{X}\right\}
$$

This formalizes the notion that locally to $0, f$ is $p$-times differentiable with the $p$ th derivative at zero bounded by $p ! C$. Sacks and Ylvisaker (1978) and Cheng et al. (1997) considered minimax MSE estimation of $f(0)$ in this class when 0 is a boundary point. Their results formally justify using local polynomial regression to estimate the RD parameter. This class does not impose any smoothness of $f$ away from cutoff, which may be too conservative

\footnotetext{
${ }^{1}$ This assumption is made to deliver finite-sample results - when the distribution of $u_{i}$ is unknown, with unknown conditional variance, we show in Supplemental Appendix E that these results lead to analogous uniform-in- $f$ asymptotic results.
} 
in applications. We consider inference under global smoothness in Armstrong and Kolesár (2016b), where we show that for the $p=2$ case, the resulting CIs are about $10 \%$ tighter in large samples (see also Supplemental Appendix C.2 for a Monte Carlo study under global smoothness).

\section{$2.1 \quad$ Optimal CIs}

For ease of exposition, we focus in this subsection on the case $p=1$, so that the parameter space is given by $\mathcal{F}=\mathcal{F}_{R D T, 1}(C)$, and assume that the errors are homoskedastic, $\sigma^{2}\left(x_{i}\right)=\sigma^{2}$. In Section 2.2, we discuss implementation of the CIs in the general case where $p \geq 1$.

Consider first the problem of constructing one-sided CIs for $L f$. In particular, consider the problem of constructing CIs $[\hat{c}, \infty)$ that minimize the maximum $\beta$ th quantile of excess length, $\sup _{f \in \mathcal{F}} q_{f, \beta}(L f-\hat{c})$, where $q_{f, \beta}$ denotes the $\beta$ th quantile of the excess length $L f-$ $\hat{c}$. We show in Section 3.3 that such CIs can be obtained by inverting tests of the null hypothesis $H_{0}: f_{+}(0)-f_{-}(0) \leq L_{0}$ that maximize their minimum power under the alternative $H_{1}: f_{+}(0)-f_{-}(0) \geq L_{0}+2 b$, where the half-distance $b$ to the alternative is calibrated so that the minimum power of these tests equals $\beta$.

To construct such a test, note that if we set $\mu=\left(f\left(x_{1}\right), \ldots, f\left(x_{n}\right)\right)^{\prime}$, and $Y=\left(y_{1}, \ldots, y_{n}\right)^{\prime}$, we can view the testing problem as an $n$-variate normal mean problem $Y \sim \mathcal{N}\left(\mu, \sigma^{2} I_{n}\right)$, in which the vector of means $\mu$ is constrained to take values in the convex sets $M_{0}=\left\{\left(f\left(x_{1}\right), \ldots\right.\right.$, $\left.\left.f\left(x_{n}\right)\right)^{\prime}: f \in \mathcal{F}, f_{+}(0)-f_{-}(0) \leq L_{0}\right\}$ under the null, and $M_{1}=\left\{\left(g\left(x_{1}\right), \ldots, g\left(x_{n}\right)\right)^{\prime}: g \in\right.$ $\left.\mathcal{F}, g_{+}(0)-g_{-}(0) \geq L_{0}+2 b\right\}$ under the alternative. The convexity of the null and alternative sets implies that this testing problem has a simple solution: by Lemma A.2, the minimax test is given by the uniformly most powerful test of the simple null $\mu=\mu_{0}^{*}$ against the simple alternative $\mu=\mu_{1}^{*}$, where $\mu_{0}^{*}$ and $\mu_{1}^{*}$ minimize the Euclidean distance between the null and alternative sets $M_{0}$ and $M_{1}$, and thus represent points in $M_{0}$ and $M_{1}$ that are hardest to distinguish. By the Neyman-Pearson lemma, such test rejects for large values of $\left(\mu_{1}^{*}-\mu_{0}^{*}\right)^{\prime} Y$. Because by Lemma A.2, this test controls size over all of $M_{0}$, the points $\mu_{1}^{*}$ and $\mu_{0}^{*}$ are called "least favorable" (see Theorem 8.1.1 in Lehmann and Romano, 2005).

To compute $\mu_{0}^{*}=\left(f^{*}\left(x_{1}\right), \ldots, f^{*}\left(x_{n}\right)\right)^{\prime}$ and $\mu_{1}^{*}=\left(g^{*}\left(x_{1}\right), \ldots, g^{*}\left(x_{n}\right)\right)^{\prime}$, we thus need to find functions $f^{*}$ and $g^{*}$ that solve

$$
\left(f^{*}, g^{*}\right)=\underset{f, g \in \mathcal{F}}{\operatorname{argmin}} \sum_{i=1}^{n}\left(f\left(x_{i}\right)-g\left(x_{i}\right)\right)^{2} \quad \text { subject to } L f \leq L_{0}, L g \geq L_{0}+2 b \text {. }
$$


A simple calculation shows that the least favorable functions solving this minimization problem are given by

$$
\begin{aligned}
& g^{*}(x)=1(x \geq 0)\left(L_{0}+b\right)+C h_{+} \cdot k_{+}\left(x / h_{+}\right)-C h_{-} \cdot k_{-}\left(x / h_{-}\right), \\
& f^{*}(x)=2 \cdot 1(x \geq 0)\left(L_{0}+b\right)-g^{*}(x),
\end{aligned}
$$

where $k(u)=\max \{0,1-|u|\}$ is the triangular kernel, $k_{+}(u)=k(u) 1(u \geq 0)$ and $k_{-}(u)=$ $k(u) 1(u<0)$, and the "bandwidths" $h_{+}, h_{-}$are determined by a condition ensuring that $L g^{*} \geq L_{0}+2 b$,

$$
h_{+}+h_{-}=b / C
$$

and a condition ensuring that positive and negative observations are equally weighted,

$$
h_{+} \sum_{i=1}^{n} k_{+}\left(x_{i} / h_{+}\right)=h_{-} \sum_{i=1}^{n} k_{-}\left(x_{i} / h_{-}\right)
$$

Intuitively, to make the null and alternative hardest to distinguish, the least favorable functions $f^{*}$ and $g^{*}$ converge to each other "as quickly as possible", subject to the constraints $L f^{*} \leq L_{0}$ and $L g^{*} \geq b+L_{0}$, and the Lipschitz constraint-see Figure 1.

By working out the appropriate critical value and rearranging, we obtain that the minimax test rejects whenever

$$
\hat{L}_{h_{+}, h_{-}}-L_{0}-\operatorname{bias}_{f^{*}}\left(\hat{L}_{h_{+}, h_{-}}\right) \geq \operatorname{sd}\left(\hat{L}_{h_{+}, h_{-}}\right) z_{1-\alpha} .
$$

Here $\hat{L}_{h_{+}, h_{-}}$is a kernel estimator based on a triangular kernel and bandwidths $h_{+}$to the left and $h_{-}$to the right of the cutoff

$$
\hat{L}_{h_{+}, h_{-}}=\frac{\sum_{i=1}^{n}\left(g^{*}\left(x_{i}\right)-f^{*}\left(x_{i}\right)\right) y_{i}}{\sum_{i=1}^{n}\left(g_{+}^{*}\left(x_{i}\right)-f_{+}^{*}\left(x_{i}\right)\right)}=\frac{\sum_{i=1}^{n} k_{+}\left(x_{i} / h_{+}\right) y_{i}}{\sum_{i=1}^{n} k_{+}\left(x_{i} / h_{+}\right)}-\frac{\sum_{i=1}^{n} k_{-}\left(x_{i} / h_{-}\right) y_{i}}{\sum_{i=1}^{n} k_{-}\left(x_{i} / h_{-}\right)},
$$

$\operatorname{sd}\left(\hat{L}_{h_{+}, h_{-}}\right)=\left(\frac{\sum_{i} k_{+}\left(x_{i} / h_{+}\right)^{2}}{\left(\sum_{i} k_{+}\left(x_{i} / h_{+}\right)\right)^{2}}+\frac{\sum_{i} k_{-}\left(x_{i} / h_{-}\right)^{2}}{\left(\sum_{i} k_{-}\left(x_{i} / h_{-}\right)\right)^{2}}\right)^{1 / 2} \cdot \sigma$ is its standard deviation, $z_{1-\alpha}$ is the $1-\alpha$ quantile of a standard normal distribution, and $\operatorname{bias}_{f^{*}}\left(\hat{L}_{h_{+}, h_{-}}\right)=C \sum_{i}\left|x_{i}\right| \cdot\left(\frac{k_{+}\left(x_{i} / h_{+}\right)}{\sum_{j} k_{+}\left(x_{j} / h_{+}\right)}+\right.$ $\left.\frac{k_{-}\left(x_{i} / h_{-}\right)}{\sum_{j} k_{-}\left(x_{j} / h_{-}\right)}\right)$is the estimator's bias under $f^{*}$. The estimator $\hat{L}_{h_{+}, h_{-}}$is normally distributed with variance that does not depend on the true function $f$. Its bias, however, does depend on $f$. To control size under $H_{0}$ in finite samples, it is necessary to subtract the largest possible bias of $\hat{L}_{h}$ under the null, which obtains at $f^{*}$. Since the rejection probability of the test is decreasing in the bias, its minimum power occurs when the bias is minimal under $H_{1}$, which 
occurs at $g^{*}$, and is given by

$$
\beta=\Phi\left(2 C \sqrt{h_{+}^{2} \sum_{i} k_{+}\left(x_{i} / h_{+}\right)^{2}+h_{-}^{2} \sum_{i} k_{-}\left(x_{i} / h_{-}\right)^{2}} / \sigma-z_{1-\alpha}\right) .
$$

Since the estimator, its variance, and the non-random bias correction are all independent of the particular null $L_{0}$, the CI based on inverting these tests as $H_{0}$ varies over $\mathbb{R}$ is given by

$$
\left[\hat{c}_{\alpha, h_{+}, h_{-}}, \infty\right), \quad \text { where } \quad \hat{c}_{\alpha, h_{+}, h_{-}}=\hat{L}_{h_{+}, h_{-}}-\operatorname{bias}_{f^{*}}\left(\hat{L}_{h_{+}, h_{-}}\right)-\operatorname{sd}\left(\hat{L}_{h_{+}, h_{-}}\right) z_{1-\alpha} .
$$

This CI minimizes the $\beta$ th quantile maximum excess length with $\beta$ given by the minimax power of the tests (8). Equivalently, given a quantile $\beta$ that we wish to optimize, let $h_{+}(\beta)$ and $h_{-}(\beta)$ solve (5) and (8). The optimal CI is then given by $\left[\hat{c}_{\alpha, h_{+}(\beta), h_{-}(\beta)}, \infty\right)$, and the half-distance $b$ to the alternative of the underlying tests is determined by (4). The important feature of this CI is that the bias correction is non-random: it depends on the worst-case bias of $\hat{L}_{h_{+}(\beta), h_{-}(\beta)}$, rather than an estimate of the bias. Furthermore, it doesn't disappear asymptotically. One can show that the squared worst-case bias of $\hat{L}_{h_{+}(\beta), h_{-}(\beta)}$ and its variance are both of the order $n^{-2 / 3}$. Consequently, no CI that "undersmooths" in the sense that it is based on an estimator whose bias is of lower order than its variance can be minimax optimal asymptotically or in finite samples.

An apparent disadvantage of this CI is that it requires the researcher to choose the smoothness parameter $C$. Addressing this issue leads to "adaptive" CIs. Adaptive CIs achieve good excess length properties for a range of parameter spaces $\mathcal{F}_{R D T, 1}\left(C_{j}\right), C_{1}<$ $\cdots<C_{J}$, while maintaining coverage over their union, which is given by $\mathcal{F}_{R D T, 1}\left(C_{J}\right)$, where $C_{J}$ is some conservative upper bound on the possible smoothness of $f$. In contrast, a minimax CI only considers worst-case excess length over $\mathcal{F}_{R D T, 1}\left(C_{J}\right)$. To derive an upper bound on the scope for adaptivity, consider the problem of finding a CI that optimizes excess length over $\mathcal{F}_{R D T, 1}(0)$ (the space of functions that are constant on either side of the cutoff), while maintaining coverage over $\mathcal{F}_{R D T, 1}(C)$ for some $C>0$.

To derive the form of such CI, consider the one-sided testing problem $H_{0}: L f \leq L_{0}$ and $f \in \mathcal{F}_{R D T, 1}(C)$ against the one-sided alternative $H_{1}: f(0) \geq L_{0}+b$ and $f \in \mathcal{F}_{R D T, 1}(0)$ (so that now the half-distance to the alternative is given by $b / 2$ rather than $b$ ). This is equivalent to a multivariate normal mean problem $Y \sim \mathcal{N}\left(\mu, \sigma^{2} I_{n}\right)$, with $\mu \in M_{0}$ under the null as before, and $\mu \in \tilde{M}_{1}=\left\{\left(f\left(x_{1}\right), \ldots, f\left(x_{n}\right)\right)^{\prime}: f \in \mathcal{F}_{R D T, 1}(0), L f \geq L_{0}+b\right\}$. Since the null and alternative are convex, by the same arguments as before, the least favorable functions minimize the distance between the two sets. The minimizing functions are given by 
$\tilde{g}^{*}(x)=1(x \geq 0)\left(L_{0}+b\right)$, and $\tilde{f}^{*}=f^{*}$ (same function as before). Since $\tilde{g}^{*}-\tilde{f}^{*}=\left(g^{*}-f^{*}\right) / 2$, this leads to the same test and the same CI as before - the only difference is that we moved the half-distance to the alternative from $b$ to $b / 2$. Hence, the minimax CI that optimizes a given quantile of excess length over $\mathcal{F}_{R D T, 1}(C)$ also optimizes its excess length over the space of constant functions, but at a different quantile. Furthermore, in Section 3.3, we show that the minimax CI remains highly efficient if one compares excess length at the same quantile: in large samples, the efficiency at constant functions is $95.2 \%$. Therefore, it is not possible to "adapt" to cases in which the regression function is smoother than the least favorable function. Consequently, it is not possible to tighten the minimax CI by, say, using the data to "estimate" the smoothness parameter $C$.

A two-sided CI can be formed as $\hat{L}_{h_{+}, h_{-}} \pm\left(\operatorname{bias}_{f^{*}}\left(\hat{L}_{h_{+}, h_{-}}\right)+\operatorname{sd}\left(\hat{L}_{h_{+}, h_{-}}\right) z_{1-\alpha / 2}\right)$, thereby accounting for possible bias of $\hat{L}_{h_{+}, h_{-}}$. However, this is conservative, since the bias cannot be in both directions at once. Since the $t$-statistic $\left(\hat{L}_{h_{+}, h_{-}}-L f\right) / \operatorname{sd}\left(\hat{L}_{h_{+}, h_{-}}\right)$is normally distributed with variance one and mean at $\operatorname{most}_{\operatorname{bias}} f^{*}\left(\hat{L}_{h_{+}, h_{-}}\right) / \operatorname{sd}\left(\hat{L}_{h_{+}, h_{-}}\right)$and least $-\operatorname{bias}_{f^{*}}\left(\hat{L}_{h_{+}, h_{-}}\right) / \operatorname{sd}\left(\hat{L}_{h_{+}, h_{-}}\right)$, a nonconservative CI takes the form

$$
\hat{L}_{h_{+}, h_{-}} \pm \operatorname{sd}\left(\hat{L}_{h_{+}, h_{-}}\right) \operatorname{cv}_{\alpha}\left(\operatorname{bias}_{f^{*}}\left(\hat{L}_{h_{+}, h_{-}}\right) / \operatorname{sd}\left(\hat{L}_{h_{+}, h_{-}}\right)\right)
$$

where $\mathrm{cv}_{\alpha}(t)$ is the $1-\alpha$ quantile of the absolute value of a $\mathcal{N}(t, 1)$ distribution, which we tabulate in Table 1 . The optimal bandwidths $h_{+}$and $h_{-}$simply minimize the CI's length, $2 \operatorname{sd}\left(\hat{L}_{h_{+}, h_{-}}\right) \cdot \operatorname{cv}_{\alpha}\left(\operatorname{bias}_{f^{*}}\left(\hat{L}_{h_{+}, h_{-}}\right) / \operatorname{sd}\left(\hat{L}_{h_{+}, h_{-}}\right)\right)$. It can be shown that the solution satisfies (5), so choosing optimal bandwidths is a one-dimensional optimization problem. Since the length doesn't depend on the data $Y$, minimizing it does not impact the coverage properties of the CI. This CI corresponds to the optimal affine fixed-length CI, as defined in Donoho (1994). Since the length of the CI doesn't depend on the data $Y$, it cannot be adaptive. In Section 3.4 we derive a sharp efficiency bound that shows that, similar to the one-sided case, these CIs are nonetheless highly efficient relative to variable-length CIs that optimize their length at smooth functions.

The key to these non-adaptivity results is that the class $\mathcal{F}$ is centrosymmetric (i.e. $f \in \mathcal{F}$ implies $-f \in \mathcal{F}$ ) and convex. For adaptivity to be possible, it is necessary (but perhaps not sufficient) to impose shape restrictions like monotonicity, or non-convexity of $\mathcal{F}$. 


\subsection{Practical implementation}

We now discuss some practical issues that arise when implementing optimal CIs. ${ }^{2}$ To describe the form of the optimal CIs for general $p \geq 1$, consider first the problem of constructing CIs based on a linear estimator of the form

$$
\hat{L}_{h_{+}, h_{-}}=\sum_{i=1}^{n} w_{+}\left(x_{i}, h_{+}\right) y_{i}-\sum_{i=1}^{n} w_{-}\left(x_{i}, h_{-}\right) y_{i}
$$

where $h_{+}, h_{-}$are smoothing parameters, and the weights satisfy $w_{+}\left(-x, h_{+}\right)=w_{-}\left(x, h_{-}\right)=$ 0 for $x \geq 0$. The estimator $\hat{L}_{h_{+}, h_{-}}$is normally distributed with variance $\operatorname{sd}\left(\hat{L}_{h_{+}, h_{-}}\right)^{2}=$ $\sum_{i=1}^{n}\left(w_{+}\left(x_{i}, h_{+}\right)+w_{-}\left(x_{i}, h_{-}\right)\right)^{2} \sigma^{2}\left(x_{i}\right)$, which does not depend on $f$. A simple argument (see Supplemental Appendix E) shows that largest possible bias of $\hat{L}_{h_{+}, h_{-}}$over the parameter space $\mathcal{F}_{R D T, p}(C)$ is given by

$$
\overline{\operatorname{bias}}_{\mathcal{F}_{R D T, p}(C)}\left(\hat{L}_{h_{+}, h_{-}}\right)=C \sum_{i=1}^{n}\left|w_{+}\left(x_{i}, h_{+}\right)+w_{-}\left(x_{i}, h_{-}\right)\right| \cdot\left|x_{i}\right|^{p},
$$

provided that the weights are such that $\hat{L}_{h_{+}, h_{-}}$is unbiased for $f$ that takes the form of a $(p-1)$ th order polynomial on either side of cutoff (otherwise the worst-case bias will be infinite). By arguments as in Section 2.1, one can construct one- and two-sided CIs based on $\hat{L}_{h_{+}, h_{-}}$as

$$
\left[c\left(\hat{L}_{h_{+}, h_{-}}\right), \infty\right) \quad c\left(\hat{L}_{h_{+}, h_{-}}\right)=\hat{L}_{h_{+}, h_{-}}-\overline{\operatorname{bias}}_{\mathcal{F}_{R D T, p}(C)}\left(\hat{L}_{h_{+}, h_{-}}\right)-\operatorname{sd}\left(\hat{L}_{h_{+}, h_{-}}\right) z_{1-\alpha},
$$

and

$$
\hat{L}_{h_{+}, h_{-}} \pm \operatorname{cv}_{\alpha}\left(\overline{\operatorname{bias}}_{\mathcal{F}_{R D T, p}(C)}\left(\hat{L}_{h_{+}, h_{-}}\right) / \operatorname{sd}\left(\hat{L}_{h_{+}, h_{-}}\right)\right) \cdot \operatorname{sd}\left(\hat{L}_{h_{+}, h_{-}}\right) .
$$

The problem of constructing optimal two- and one- sided CIs can be cast as a problem of finding weights $w_{+}, w_{-}$and smoothing parameters $h_{+}$and $h_{-}$that lead to CIs with the shortest length, and smallest worst-case $\beta$ quantile of excess length, respectively. The solution to this problem follows from a generalization of results in Sacks and Ylvisaker (1978). The optimal weights $w_{+}$and $w_{-}$are given by a solution to a system of $2(p-1)$ equations, described in Supplemental Appendix E. When $p=1$, they reduce to the weights $w_{+}\left(x_{i}, h_{+}\right)=k_{+}\left(x_{i} / h_{+}\right) / \sum_{i} k_{+}\left(x_{i} / h_{+}\right)$and $w_{-}\left(x_{i}, h_{-}\right)=k_{-}\left(x_{i} / h_{+}\right) / \sum_{i} k_{-}\left(x_{i} / h_{+}\right)$, where $k_{+}\left(x_{i}\right)=k\left(x_{i}\right) 1\left(x_{i} \geq 0\right)$ and $k_{-}\left(x_{i}\right)=k\left(x_{i}\right) 1\left(x_{i}<0\right)$, and $k(u)=\max \{0,1-|u|\}$ is a

\footnotetext{
${ }^{2}$ An R package implementing these CIs is available at https://github.com/kolesarm/RDHonest.
} 
triangular kernel. This leads to the triangular kernel estimator (7). For $p>1$, the optimal weights depend on the empirical distribution of the running variable $x_{i}$.

An alternative to using the optimal weights is to use a local polynomial estimator of order $p-1$, with kernel $k$ and bandwidths $h_{-}$and $h_{+}$to the left and to the right of the cutoff. This leads to weights of the form

$$
w_{+}\left(x_{i}, h_{+}\right)=e_{1}^{\prime}\left(\sum_{i} k_{+}\left(x_{i} / h_{+}\right) r_{i} r_{i}^{\prime}\right)^{-1} \sum_{i} k_{+}\left(x_{i} / h_{+}\right) r_{i}
$$

and similarly for $w_{-}\left(x_{i}, h_{-}\right)$, where $r_{i}=\left(1, x_{i}, \ldots, x_{i}^{p-1}\right)$ and $e_{1}$ is the first unit vector. Using the efficiency bounds we develop in Section 3, it can be shown that, provided that the bandwidths $h_{+}$and $h_{-}$to the right and to the left of the cutoff are appropriately chosen, in many cases the resulting CIs are highly efficient. In particular, for $p=2$, using the local linear estimator with the triangular kernel turns out to lead to near-optimal CIs (see Section 4).

Thus, given smoothness constants $C$ and $p$, one can construct optimal or near-optimal CIs as follows:

1. Form a preliminary estimator of the conditional variance $\hat{\sigma}\left(x_{i}\right)$. We recommend using the estimator $\hat{\sigma}^{2}\left(x_{i}\right)=\hat{\sigma}_{+}^{2}(0) 1(x \geq 0)+\hat{\sigma}_{-}^{2}(0) 1(x<0)$ where $\hat{\sigma}_{+}^{2}(0)$ and $\hat{\sigma}_{-}^{2}(0)$ are estimates of $\lim _{x \downarrow 0} \sigma^{2}(x)$ and $\lim _{x \uparrow 0} \sigma^{2}(x)$ respectively. ${ }^{3}$

2. Given smoothing parameters $h_{+}$and $h_{-}$, compute the weights $w_{+}$and $w_{-}$using either (14) (for local polynomial estimator), or by solving the system of equations given in Supplemental Appendix E (for the optimal estimator). Compute the worst case bias (11), and estimate the variance as $\widehat{\operatorname{sd}}\left(\hat{L}_{h_{+}, h_{-}}\right)^{2}=\sum_{i}\left(w_{+}\left(x_{i}, h_{+}\right)+w_{-}\left(x_{i}, h_{-}\right)\right)^{2} \hat{\sigma}^{2}\left(x_{i}\right)$.

3. Find the smoothing parameters $h_{+}^{*}$ and $h_{-}^{*}$ that minimize the $\beta$-quantile of excess length

$$
2 \overline{\operatorname{bias}}_{\mathcal{F}_{R D T, p}(c)}\left(\hat{L}_{h_{+}, h_{-}}\right)+\operatorname{sd}\left(\hat{L}_{h_{+}, h_{-}}\right)\left(z_{1-\alpha}+z_{\beta}\right) .
$$

for a given $\beta$. The choice $\beta=0.8$, corresponds to a benchmark used in statistical power analysis (see Cohen, 1988). For two-sided CIs, minimize the length

$$
2 \widehat{\operatorname{sd}}\left(\hat{L}_{h_{+}, h_{-}}\right) \mathrm{cv}_{\alpha}\left(\overline{\operatorname{bias}}_{\mathcal{F}_{R D T, p}(C)}\left(\hat{L}_{h_{+}, h_{-}}\right) / \widehat{\operatorname{sd}}\left(\hat{L}_{h_{+}, h_{-}}\right)\right)
$$

\footnotetext{
${ }^{3}$ In the empirical application in Section 4, we use estimates based on local linear regression residuals.
} 
4. Construct the CI using (12) (for one-sided CIs), or (13) (for two-sided CIs), based on $\hat{L}_{h_{+}^{*}, h_{-}^{*}}$, with $\widehat{\operatorname{sd}}\left(\hat{L}_{h_{+}^{*}, h_{-}^{*}}\right)$ in place of the (infeasible) true standard deviation.

Remark 2.1. The variance estimator in step 1 leads to asymptotically valid and optimal inference even when $\sigma^{2}(x)$ is non-constant, so long as it is smooth on either side of the cutoff. However, finite-sample properties of the resulting CI may not be good if heteroskedasticity is important for the sample size at hand. We therefore recommend using the variance estimator

$$
\widehat{\operatorname{sd}}_{\text {robust }}\left(\hat{L}_{h_{+}^{*}, h_{-}^{*}}\right)^{2}=\sum_{i=1}^{n}\left(w_{+}\left(x_{i}, h_{+}\right)+w_{-}\left(x_{i}, h_{-}\right)\right)^{2} \hat{u}_{i}^{2}
$$

instead of $\widehat{\operatorname{sd}}\left(\hat{L}_{h_{+}^{*}, h_{-}^{*}}\right)$ in step 4 , where $\hat{u}_{i}^{2}$ is an estimate of $\sigma^{2}\left(x_{i}\right)$. When using local polynomial regression, one can set $\hat{u}_{i}$ to the $i$ th regression residual, in which case (17) reduces to the usual Eicker-Huber-White estimator. Alternatively, one can use the nearest-neighbor estimator (Abadie and Imbens, 2006) $\hat{u}_{i}^{2}=\frac{J}{J+1}\left(Y_{i}-J^{-1} \sum_{\ell=1}^{J} Y_{j_{\ell}(i)}\right)^{2}$, where $j_{\ell}(i)$ is the $\ell$ th closest unit to $i$ among observations on the same side of the cutoff, and $J \geq 1$ (we use $J=3$ in the application in Section 4, following Calonico et al., 2014). This mirrors the common practice of assuming homoskedasticity to compute the optimal weights, but allowing for heteroskedasticity when performing inference, such as using OLS in the linear regression model (which is efficient under homoskedasticity) along with heteroskedasticity-robust standard errors.

Remark 2.2. If one is interested in estimation, rather than inference, one can choose $h_{+}$ and $h_{-}$that minimize the worst-case mean-squared error (MSE) $\overline{\operatorname{bias}}_{\mathcal{F}_{R D T, p}(C)}\left(\hat{L}_{h_{+}, h_{-}}\right)^{2}+$ $\operatorname{sd}\left(\hat{L}_{h_{+}, h_{-}}\right)^{2}$ instead of the CI criteria in step 3. One can form a CI around this estimator by simply following step 4 with this choice of $h_{+}$and $h_{-}$. In the application in Section 4 , we find that little efficiency is lost by using MSE-optimal smoothing parameters, relative to using $h_{+}$and $h_{-}$that minimize the CI length (16). Interestingly, we find that smoothing parameters that minimize the CI length actually oversmooth slightly relative to the MSE optimal smoothing parameters. We generalize these findings in an asymptotic setting in Armstrong and Kolesár (2016b).

Remark 2.3. Often, a set of covariates $z_{i}$ will be available that does not depend on the treatment, but that may be correlated with the outcome variable $y_{i}$. If the parameter of interest is still the average treatment effect for units with $x_{i}=0$, one can simply ignore these covariates. Alternatively, to gain additional precision, as suggested in Calonico et al. (2017), one can run a local polynomial regression, but with the covariates added linearly. In 
Appendix B, we show that this approach is near-optimal if one places smoothness assumptions on the conditional mean of $\tilde{y}_{i}$ given $x_{i}$, where $\tilde{y}_{i}$ is the outcome with the effect of $z_{i}$ partialled out. If one is interested in the treatment effect as a function of $z$ (with $x$ still set to zero), one can use our general framework by considering the model $y_{i}=f\left(x_{i}, z_{i}\right)+u_{i}$, specifying a smoothness class for $f$, and constructing CIs for $\lim _{x \downarrow 0} f(x, z)-\lim _{x \uparrow 0} f(x, z)$ for different values of $z$. See Appendix B for details.

A final consideration in implementing these CIs in practice is the choice of the smoothness constants $C$ and $p$. The choice of $p$ depends on the order of the derivative the researcher wishes to bound. Since much of empirical practice in RD is justified by asymptotic MSE optimality results for $\mathcal{F}_{R D T, 2}(C)$ (in particular, this class justifies the use of local linear estimators), we recommend $p=2$ as a default choice. For $C$, generalizations of the nonadaptivity results described in Section 2.1 show that the researcher must choose $C$ a priori, rather than attempting to use the data to choose $C$. To assess the sensitivity of the results to different smoothness assumptions on $f$, we recommend considering a range of plausible choices for $C$. We implement this approach for our empirical application in Section 4.

\section{General characterization of optimal procedures}

We consider the following setup and notation, much of which follows Donoho (1994). We observe data $Y$ of the form

$$
Y=K f+\sigma \varepsilon
$$

where $f$ is known to lie in a convex subset $\mathcal{F}$ of a vector space, and $K: \mathcal{F} \rightarrow \mathcal{Y}$ is a linear operator between $\mathcal{F}$ and a Hilbert space $\mathcal{Y}$. We denote the inner product on $\mathcal{Y}$ by $\langle\cdot, \cdot\rangle$, and the norm by $\|\cdot\|$. The error $\varepsilon$ is standard Gaussian with respect to this inner product: for any $g \in \mathcal{Y},\langle\varepsilon, g\rangle$ is normal with $E\langle\varepsilon, g\rangle=0$ and $\operatorname{var}(\langle\varepsilon, g\rangle)=\|g\|^{2}$. We are interested in constructing a confidence set for a linear functional $L f$.

The RD model (1) fits into this setup by setting $Y=\left(y_{1} / \sigma\left(x_{1}\right), \ldots, y_{n} / \sigma\left(x_{n}\right)\right)^{\prime}, \mathcal{Y}=\mathbb{R}^{n}$, $K f=\left(f\left(x_{1}\right) / \sigma\left(x_{1}\right), \ldots, f\left(x_{n}\right) / \sigma\left(x_{n}\right)\right)^{\prime}, L f=\lim _{x \downarrow 0} f(x)-\lim _{x \uparrow 0} f(x)$ and $\langle x, y\rangle$ given by the Euclidean inner product $x^{\prime} y$. As we discuss in detail in Supplemental Appendix D.1, our setup covers a number of other important models, including average treatment effects under unconfoundedness, the partly linear model, constraints on the sign or magnitude of parameters in the linear regression model, and other parametric models. 


\subsection{Performance criteria}

Let us now define the performance criteria that we use to evaluate confidence sets for $L f$. A set $\mathcal{C}=\mathcal{C}(Y)$ is called a $100 \cdot(1-\alpha) \%$ confidence set for $L f$ if $\inf _{f \in \mathcal{F}} P_{f}(L f \in \mathcal{C}) \geq 1-\alpha$. We denote the collection of all $100 \cdot(1-\alpha) \%$ confidence sets by $\mathcal{I}_{\alpha}$.

We can compare performance of confidence sets at a particular $f \in \mathcal{F}$ using expected length, $E_{f} \lambda(\mathcal{C})$, where $\lambda$ is Lebesgue measure. Allowing confidence sets to have arbitrary form may make them difficult to interpret or even compute. One way of avoiding this is to restrict attention to confidence sets that take the form of a fixed-length confidence interval (CI), an interval of the form $[\hat{L}-\chi, \hat{L}+\chi]$ for some estimate $\hat{L}$ and nonrandom $\chi$ (for instance, in the RD model (1), $\chi$ may depend on the running variable $x_{i}$ and $\sigma^{2}\left(x_{i}\right)$, but not on $\left.y_{i}\right)$. Let

$$
\chi_{\alpha}(\hat{L})=\min \left\{\chi: \inf _{f \in \mathcal{F}} P_{f}(|\hat{L}-L f| \leq \chi) \geq 1-\alpha\right\}
$$

denote the half-length of the shortest fixed-length $100 \cdot(1-\alpha) \%$ CI centered around an estimator $\hat{L}$. Fixed-length CIs are easy to compare: one simply prefers the one with the shortest half-length. On the other hand, their length cannot "adapt" to reflect greater precision for different functions $f \in \mathcal{F}$. To address this concern, in Section 3.4, we compare the length of fixed-length CIs to sharp bounds on the optimal expected length $\inf _{\mathcal{C} \in \mathcal{I}_{\alpha}} E_{f}(\mathcal{C})$.

If $\mathcal{C}$ is restricted to take the form of a one-sided $\mathrm{CI}[\hat{c}, \infty)$, we cannot use expected length as a criterion. We therefore measure performance at a particular parameter $f$ using the $\beta$ th quantile of their excess length $L f-\hat{c}$, which we denote by $q_{f, \beta}(L f-\hat{c})$. To measure performance globally over some set $\mathcal{G}$, we use the maximum $\beta$ th quantile of the excess length,

$$
q_{\beta}(\hat{c}, \mathcal{G})=\sup _{g \in \mathcal{G}} q_{g, \beta}(L g-\hat{c}) .
$$

If $\mathcal{G}=\mathcal{F}$, minimizing $q_{\beta}(\hat{c}, \mathcal{F})$ over one-sided CIs in the set $\mathcal{I}_{\alpha}$ gives minimax excess length. If $\mathcal{G} \subset \mathcal{F}$ is a class of smoother functions, minimizing $q_{\beta}(\hat{c}, \mathcal{G})$ yields CIs that direct power: they achieve good performance when $f$ is smooth, while maintaining coverage over all of $\mathcal{F}$. A CI that achieves good performance over multiple classes $\mathcal{G}$ is said to be "adaptive" over these classes. In Section 3.3, we give sharp bounds on (19) for a single class $\mathcal{G}$, which gives a benchmark for adapting over multiple classes (cf. Cai and Low, 2004a). 


\subsection{Affine estimators and optimal bias-variance tradeoff}

Many popular estimators are linear functions of the outcome variable $Y$, and we will see below that optimal or near-optimal CIs are based on estimators of this form. In the general framework (18), linear estimators take the form $\langle w, Y\rangle$ for some non-random $w \in \mathcal{Y}$, which simplifies to (10) in the RD model. It will be convenient to allow for a recentering by some constant $a \in \mathbb{R}$, which leads to an affine estimator $\hat{L}=a+\langle w, Y\rangle$.

For any estimator $\hat{L}$, let $\overline{\operatorname{bias}}_{\mathcal{G}}(\hat{L})=\sup _{f \in \mathcal{G}} E_{f}(\hat{L}-L f)$ and $\underline{\operatorname{bias}}_{\mathcal{G}}(\hat{L})=\inf _{f \in \mathcal{G}} E_{f}(\hat{L}-L f)$. An affine estimator $\hat{L}=a+\langle w, Y\rangle$ follows a normal distribution with mean $E_{f} \hat{L}=a+$ $\langle w, K f\rangle$ and variance $\operatorname{var}(\hat{L})=\|w\|^{2} \sigma^{2}$, which does not depend on $f$. Thus, the set of possible distributions for $\hat{L}-L f$ as $f$ varies over a given convex set $\mathcal{G}$ is given by the set of normal distributions with variance $\|w\|^{2} \sigma^{2}$ and mean between $\underline{\operatorname{bias}}_{\mathcal{G}}(\hat{L})$ and $\overline{\operatorname{bias}}_{\mathcal{G}}(\hat{L})$. It follows that a one-sided CI based on an affine estimator $\hat{L}$ is given by

$$
[\hat{c}, \infty) \quad \hat{c}=\hat{L}-\overline{\operatorname{bias}}_{\mathcal{F}}(\hat{L})-\operatorname{sd}(\hat{L}) z_{1-\alpha}
$$

with $z_{1-\alpha}$ denoting the $1-\alpha$ quantile of a standard normal distribution, and that its worstcase $\beta$ th quantile excess length over a convex class $\mathcal{G}$ is

$$
q_{\beta}(\hat{c}, \mathcal{G})=\overline{\operatorname{bias}}_{\mathcal{F}}(\hat{L})-\underline{\operatorname{bias}}_{\mathcal{G}}(\hat{L})+\operatorname{sd}(\hat{L})\left(z_{1-\alpha}+z_{\beta}\right) .
$$

The shortest fixed-length CI centered at the affine estimator $\hat{L}$ is given by

$$
\hat{L} \pm \chi_{\alpha}(\hat{L}), \quad \chi_{\alpha}(\hat{L})=\operatorname{cv}_{\alpha}\left(\frac{\max \left\{\left|\overline{\operatorname{bias}}_{\mathcal{F}}(\hat{L})\right|,\left|\underline{\operatorname{bias}}_{\mathcal{F}}(\hat{L})\right|\right\}}{\operatorname{sd}(\hat{L})}\right) \cdot \operatorname{sd}(\hat{L})
$$

where $\mathrm{cv}_{\alpha}(t)$ is the $1-\alpha$ quantile of the absolute value of a $\mathcal{N}(t, 1)$ random variable, as tabulated in Table 1.

The fact that optimal CIs turn out to be based on affine estimators reduces the derivation of optimal CIs to bias-variance calculations: since the performance of CIs based on affine estimators depends only on the variance and worst-case bias, one simply minimizes worstcase bias subject to a bound on variance, and then trades off bias and variance in a way that is optimal for the given criterion. The main tool for doing this is the ordered modulus of continuity between $\mathcal{F}$ and $\mathcal{G}$ (Cai and Low, 2004a),

$$
\omega(\delta ; \mathcal{F}, \mathcal{G})=\sup \{L g-L f:\|K(g-f)\| \leq \delta, f \in \mathcal{F}, g \in \mathcal{G}\}
$$


for any sets $\mathcal{F}$ and $\mathcal{G}$ with a non-empty intersection (so that the set over which the supremum is taken is non-empty). When $\mathcal{G}=\mathcal{F}, \omega(\delta ; \mathcal{F}, \mathcal{F})$ is the (single-class) modulus of continuity over $\mathcal{F}$ (Donoho and Liu, 1991), and we denote it by $\omega(\delta ; \mathcal{F})$. The ordered modulus $\omega(\cdot ; \mathcal{F}, \mathcal{G})$ is concave, which implies that the superdifferential at $\delta$ (the set of slopes of tangent lines at $(\delta, \omega(\delta ; \mathcal{F}, \mathcal{G})))$ is nonempty for any $\delta>0$. Throughout the paper, we let $\omega^{\prime}(\delta ; \mathcal{F}, \mathcal{G})$ denote an (arbitrary unless otherwise stated) element in this set. Typically, $\omega(\cdot ; \mathcal{F}, \mathcal{G})$ is differentiable, in which case $\omega^{\prime}(\delta ; \mathcal{F}, \mathcal{G})$ is defined uniquely as the derivative at $\delta$. We use $g_{\delta, \mathcal{F}, \mathcal{G}}^{*}$ and $f_{\delta, \mathcal{F}, \mathcal{G}}^{*}$ to denote a solution to the ordered modulus problem (assuming it exists), and $f_{M, \delta, \mathcal{F}, \mathcal{G}}^{*}=\left(f_{\delta, \mathcal{F}, \mathcal{G}}^{*}+g_{\delta, \mathcal{F}, \mathcal{G}}^{*}\right) / 2$ to denote the midpoint. ${ }^{4}$

We will show that optimal decision rules will in general depend on the data $Y$ through an affine estimator of the form

$$
\hat{L}_{\delta, \mathcal{F}, \mathcal{G}}=L f_{M, \delta, \mathcal{F}, \mathcal{G}}^{*}+\frac{\omega^{\prime}(\delta ; \mathcal{F}, \mathcal{G})}{\delta}\left\langle K\left(g_{\delta, \mathcal{F}, \mathcal{G}}^{*}-f_{\delta, \mathcal{F}, \mathcal{G}}^{*}\right), Y-K f_{M, \delta, \mathcal{F}, \mathcal{G}}^{*}\right\rangle
$$

with $\delta$ and $\mathcal{G}$ depending on the optimality criterion. When $\mathcal{F}=\mathcal{G}$, we denote the estimator $\hat{L}_{\delta, \mathcal{F}, \mathcal{F}}$ by $\hat{L}_{\delta, \mathcal{F}}$. When the sets $\mathcal{F}$ and $\mathcal{G}$ are clear from the context, we use $\omega(\delta), \hat{L}_{\delta}, f_{\delta}^{*}, g_{\delta}^{*}$ and $f_{M, \delta}^{*}$ in place of $\omega(\delta ; \mathcal{F}, \mathcal{G}), \hat{L}_{\delta, \mathcal{F}, \mathcal{G}}, f_{\delta, \mathcal{F}, \mathcal{G}}^{*}, g_{\delta, \mathcal{F}, \mathcal{G}}^{*}$ and $f_{M, \delta, \mathcal{F}, \mathcal{G}}^{*}$ to avoid notational clutter.

As we show in Lemma A.1 in the Appendix, a useful property of $\hat{L}_{\delta, \mathcal{F}, \mathcal{G}}$ is that its maximum bias over $\mathcal{F}$ and minimum bias over $\mathcal{G}$ are attained at $f_{\delta}^{*}$ and $g_{\delta}^{*}$, respectively, and are given by

$$
\overline{\operatorname{bias}}_{\mathcal{F}}\left(\hat{L}_{\delta, \mathcal{F}, \mathcal{G}}\right)=-\underline{\operatorname{bias}}_{\mathcal{G}}\left(\hat{L}_{\delta, \mathcal{F}, \mathcal{G}}\right)=\frac{1}{2}\left(\omega(\delta ; \mathcal{F}, \mathcal{G})-\delta \omega^{\prime}(\delta ; \mathcal{F}, \mathcal{G})\right)
$$

Its standard deviation equals $\operatorname{sd}\left(\hat{L}_{\delta, \mathcal{F}, \mathcal{G}}\right)=\sigma \omega^{\prime}(\delta ; \mathcal{F}, \mathcal{G})$, and doesn't depend on $f$. As remarked by Cai and Low (2004b), no estimator can simultaneously achieve lower maximum bias over $\mathcal{F}$, higher minimum bias over $\mathcal{G}$, and lower variance than the estimators in the class $\left\{\hat{L}_{\delta, \mathcal{F}, \mathcal{G}}\right\}_{\delta>0}$. Estimators $(23)$ can thus be used to optimally trade off various levels of bias and variance.

A condition that will play a central role in bounding the gains from directing power at smooth functions is centrosymmetry. We say that a class $\mathcal{F}$ is centrosymmetric if $f \in \mathcal{F} \Longrightarrow$ $-f \in \mathcal{F}$. Under centrosymmetry, the functions that solve the single-class modulus problem

\footnotetext{
${ }^{4}$ See Supplemental Appendix D.2 for sufficient conditions for differentiability and a discussion of the non-differentiable case. Regarding existence of a solution to the modulus problem, we verify this directly for our RD application in Supplemental Appendix E.2; see also Donoho (1994), Lemma 2 for a general set of sufficient conditions.
} 
can be taken to satisfy $g_{\delta}^{*}=-f_{\delta}^{*}$, and the modulus is given by

$$
\omega(\delta ; \mathcal{F})=\sup \{2 L f:\|K f\| \leq \delta / 2, f \in \mathcal{F}\}
$$

Since $f_{\delta}^{*}=-g_{\delta}^{*}, f_{M, \delta}^{*}$ is the zero function and $\hat{L}_{\delta, \mathcal{F}}$ is linear:

$$
\hat{L}_{\delta, \mathcal{F}}=\frac{2 \omega^{\prime}(\delta ; \mathcal{F})}{\delta}\left\langle K g_{\delta}^{*}, Y\right\rangle
$$

In the RD model (1) the class $\mathcal{F}_{R D T, p}(C)$ is centrosymmetric, and the estimator $\hat{L}_{\delta, \mathcal{F}_{R D T, p}(C)}$ takes the form $\hat{L}_{h_{+}, h_{-}}$given in (10) for a certain class of weights $w_{+}\left(x, h_{+}\right)$and $w_{-}\left(x, h_{-}\right)$, with the smoothing parameters $h_{+}$and $h_{-}$both determined by $\delta$ (see Supplemental Appendix E).

\subsection{Optimal one-sided CIs}

Given $\beta$, a one-sided CI that minimizes (19) among all one-sided CIs with level $1-\alpha$ is based on $\hat{L}_{\delta_{\beta} ; \mathcal{F}, \mathcal{G}}$, where $\delta_{\beta}=\sigma\left(z_{\beta}+z_{1-\alpha}\right)$.

Theorem 3.1. Let $\mathcal{F}$ and $\mathcal{G}$ be convex with $\mathcal{G} \subseteq \mathcal{F}$, and suppose that $f_{\delta}^{*}$ and $g_{\delta}^{*}$ achieve the ordered modulus at $\delta$ with $\left\|K\left(f_{\delta}^{*}-g_{\delta}^{*}\right)\right\|=\delta$. Let

$$
\hat{c}_{\alpha, \delta, \mathcal{F}, \mathcal{G}}=\hat{L}_{\delta, \mathcal{F}, \mathcal{G}}-\overline{\operatorname{bias}}_{\mathcal{F}}\left(\hat{L}_{\delta, \mathcal{F}, \mathcal{G}}\right)-z_{1-\alpha} \sigma \omega^{\prime}(\delta ; \mathcal{F}, \mathcal{G})
$$

Then $\hat{c}_{\alpha, \delta, \mathcal{F}, \mathcal{G}}$ minimizes $q_{\beta}(\hat{c}, \mathcal{G})$ for $\beta=\Phi\left(\delta / \sigma-z_{1-\alpha}\right)$ among all one-sided $1-\alpha$ CIs, where $\Phi$ denotes the standard normal cdf. The minimum coverage is taken at $f_{\delta}^{*}$ and equals $1-\alpha$. All quantiles of excess length are maximized at $g_{\delta}^{*}$. The worst case $\beta$ th quantile of excess length is $q_{\beta}\left(\hat{c}_{\alpha, \delta, \mathcal{F}, \mathcal{G}}, \mathcal{G}\right)=\omega(\delta ; \mathcal{F}, \mathcal{G})$.

Since the worst-case bias of $\hat{L}_{\delta, \mathcal{F}, \mathcal{G}}$ is given by $(24)$, and its standard deviation equals $\sigma \omega^{\prime}(\delta ; \mathcal{F}, \mathcal{G})$, it can be seen that $\hat{c}_{\alpha, \delta, \mathcal{F}, \mathcal{G}}$ takes the form given in $(20)$, and its worst-case excess length follows $(21)$. The assumption that the modulus is achieved with $\left\|K\left(f_{\delta}^{*}-g_{\delta}^{*}\right)\right\|=\delta$ rules out degenerate cases: if $\left\|K\left(f_{\delta}^{*}-g_{\delta}^{*}\right)\right\|<\delta$, then relaxing this constraint does not increase the modulus, which means that $\omega^{\prime}(\delta ; \mathcal{F}, \mathcal{G})=0$ and the optimal CI does not depend on the data.

Implementing the CI from Theorem 3.1 requires the researcher to choose a quantile $\beta$ to optimize, and to choose the set $\mathcal{G}$. There are two natural choices for $\beta$. If the objective is to optimize the performance of the CI "on average", then optimizing the median excess length 
$(\beta=0.5)$ is a natural choice. Since for any CI $[\hat{c}, \infty)$ such that $\hat{c}$ is affine in the data $Y$, the median and expected excess lengths coincide, and since $\hat{c}_{\alpha, \delta, \mathcal{F}, \mathcal{G}}$ is affine in the data, setting $\beta=0.5$ also has the advantage that it minimizes the expected excess length among affine CIs. Alternatively, if the CI is being computed as part of a power analysis, then setting $\beta=0.8$ is natural, as, under conditions given in Supplemental Appendix D.2, it translates directly to statements about $80 \%$ power, a standard benchmark in such analyses (Cohen, 1988).

For the set $\mathcal{G}$, there are two leading choices. First, setting $\mathcal{G}=\mathcal{F}$ yields minimax CIs:

Corollary 3.1 (One-sided minimax CIs). Let $\mathcal{F}$ be convex, and suppose that $f_{\delta}^{*}$ and $g_{\delta}^{*}$ achieve the single-class modulus at $\delta$ with $\left\|K\left(f_{\delta}^{*}-g_{\delta}^{*}\right)\right\|=\delta$. Let

$$
\hat{c}_{\alpha, \delta, \mathcal{F}}=\hat{L}_{\delta, \mathcal{F}}-\frac{1}{2}\left(\omega(\delta ; \mathcal{F})-\delta \omega^{\prime}(\delta ; \mathcal{F})\right)-z_{1-\alpha} \sigma \omega^{\prime}(\delta ; \mathcal{F})
$$

Then, for $\beta=\Phi\left(\delta / \sigma-z_{1-\alpha}\right), \hat{c}_{\alpha, \delta, \mathcal{F}}$ minimizes the maximum $\beta$ th quantile of excess length among all $1-\alpha$ CIs for $L f$. The minimax excess length is given by $\omega(\delta ; \mathcal{F})$.

The minimax criterion may be considered overly pessimistic: it focuses on controlling the excess length under the least favorable function. This leads to the second possible choice for $\mathcal{G}$, a smaller convex class of smoother functions $\mathcal{G} \subset \mathcal{F}$. The resulting CIs will then achieve the best possible performance when $f$ is smooth, while maintaining coverage over all of $\mathcal{F}$. Unfortunately, there is little scope for improvement for such a CI when $\mathcal{F}$ is centrosymmetric. In particular, suppose that $g_{\delta, \mathcal{F}, \mathcal{G}}^{*}$ is "sufficiently smooth" relative to $\mathcal{F}$, in the sense that

$$
f-g_{\delta, \mathcal{F}, \mathcal{G}}^{*} \in \mathcal{F} \quad \text { for all } f \in \mathcal{F}
$$

Since $\mathcal{F}$ is centrosymmetric, this condition is equivalent to the requirement that the sets $\left\{f-g_{\delta, \mathcal{F}, \mathcal{G}}^{*}: f \in \mathcal{F}\right\}$ and $\mathcal{F}$ are the same. ${ }^{5}$ For instance, (27) holds if $\mathcal{G}$ contains the zero function only. In the $\mathrm{RD}$ model $(1)$ with $\mathcal{F}=\mathcal{F}_{R D T, p}(C),(27)$ holds if $\mathcal{G}=\mathcal{F}_{R D T, p}(0)$, the class of piecewise polynomial functions.

Corollary 3.2. Let $\mathcal{F}$ be centrosymmetric, and let $\mathcal{G} \subseteq \mathcal{F}$ be any convex set such that the solution to the ordered modulus problem exists and satisfies (27) with $\left\|K\left(f_{\delta_{\beta}}^{*}-g_{\delta_{\beta}}^{*}\right)\right\|=$ $\delta_{\beta}$, where $\delta_{\beta}=\sigma\left(z_{\beta}+z_{1-\alpha}\right)$. Then the one-sided $C I \hat{c}_{\alpha, \delta_{\beta}, \mathcal{F}}$ that is minimax for the $\beta$ th quantile also optimizes $q_{\tilde{\beta}}(\hat{c} ; \mathcal{G})$, where $\tilde{\beta}=\Phi\left(\left(z_{\beta}-z_{1-\alpha}\right) / 2\right)$. In particular, $\hat{c}_{\alpha, \delta_{\beta}, \mathcal{F}}$ optimizes

\footnotetext{
${ }^{5}$ We thank a referee for pointing this out.
} 
$q_{\tilde{\beta}}(\hat{c} ;\{0\})$. Moreover, the efficiency of $\hat{c}_{\alpha, \delta_{\beta}, \mathcal{F}}$ for the $\beta$ th quantile of maximum excess length over $\mathcal{G}$ is given by

$$
\frac{\inf _{\hat{c}:[\hat{c}, \infty) \in \mathcal{I}_{\alpha}} q_{\beta}(\hat{c}, \mathcal{G})}{q_{\beta}\left(\hat{c}_{\alpha, \delta_{\beta}, \mathcal{F}}, \mathcal{G}\right)}=\frac{\omega\left(\delta_{\beta} ; \mathcal{F}, \mathcal{G}\right)}{q_{\beta}\left(\hat{c}_{\alpha, \delta_{\beta}, \mathcal{F}}, \mathcal{G}\right)}=\frac{\omega\left(2 \delta_{\beta} ; \mathcal{F}\right)}{\omega\left(\delta_{\beta} ; \mathcal{F}\right)+\delta_{\beta} \omega^{\prime}\left(\delta_{\beta} ; \mathcal{F}\right)}
$$

The first part of Corollary 3.2 states that minimax CIs that optimize a particular quantile $\beta$ will also minimize the maximum excess length over $\mathcal{G}$ at a different quantile $\tilde{\beta}$. For instance, a CI that is minimax for median excess length among 95\% CIs also optimizes $\Phi\left(-z_{0.95} / 2\right) \approx 0.205$ quantile under the zero function. Vice versa, the CI that optimizes median excess length under the zero function is minimax for the $\Phi\left(2 z_{0.5}+z_{0.95}\right)=0.95$ quantile.

The second part of Corollary 3.2 gives the exact cost of optimizing the "wrong" quantile $\tilde{\beta}$. Since the one-class modulus is concave, $\delta \omega^{\prime}(\delta) \leq \omega(\delta)$, and we can lower bound the efficiency of $\hat{c}_{\alpha, \delta_{\beta}, \mathcal{F}}$ given in $(28)$ by $\omega\left(2 \delta_{\beta}\right) /\left(2 \omega\left(\delta_{\beta}\right)\right) \geq 1 / 2$. Typically, the efficiency is much higher. In particular, in the regression model (1), the one-class modulus satisfies

$$
\omega(\delta ; \mathcal{F})=n^{-r / 2} A \delta^{r}(1+o(1))
$$

for many choices of $\mathcal{F}$ and $L$, as $n \rightarrow \infty$ for some constant $A$, where $r / 2$ is the rate of convergence of the minimax root MSE. This is the case under regularity conditions in the RD model with $r=2 p /(2 p+1)$ by Lemma H.6 (see Donoho and Low, 1992, for other cases where (29) holds). In this case, (28) evaluates to $\frac{2^{r}}{1+r}(1+o(1))$, so that the asymptotic efficiency depends only on $r$. Figure 2 plots the asymptotic efficiency as a function of $r$. Since adapting to the zero function easier than adapting to any set $\mathcal{G}$ that includes it, if $\mathcal{F}$ is convex and centrosymmetric, "directing power" yields very little gain in excess length no matter how optimistic one is about where to direct it.

This result places a severe bound on the scope for adaptivity in settings in which $\mathcal{F}$ is convex and centrosymmetric: any CI that performs better than the minimax CI by more than the ratio in (28) must fail to control coverage at some $f \in \mathcal{F}$. 


\subsection{Two-sided CIs}

A fixed-length CI based on $\hat{L}_{\delta, \mathcal{F}}$ can be computed by plugging its worst-case bias (24) into $(22),{ }^{6}$

$$
\hat{L}_{\delta, \mathcal{F}} \pm \chi_{\alpha}\left(\hat{L}_{\delta, \mathcal{F}}\right), \quad \chi_{\alpha}\left(\hat{L}_{\delta, \mathcal{F}}\right)=\mathrm{cv}_{\alpha}\left(\frac{\omega(\delta ; \mathcal{F})}{2 \sigma \omega^{\prime}(\delta ; \mathcal{F})}-\frac{\delta}{2 \sigma}\right) \cdot \sigma \omega^{\prime}(\delta ; \mathcal{F})
$$

The optimal $\delta$ minimizes the half-length, $\delta_{\chi}=\operatorname{argmin}_{\delta>0} \chi_{\alpha}\left(\hat{L}_{\delta, \mathcal{F}}\right)$. It follows from Donoho (1994) that this CI is the shortest possible in the class of fixed-length CIs based on affine estimators. Just as with minimax one-sided CIs, one may worry that since its length is driven by the least favorable functions, restricting attention to fixed-length CIs may be costly when the true $f$ is smoother. The next result characterizes confidence sets that optimize expected length at a single function $g$, and thus bounds the possible performance gain.

Theorem 3.2. Let $g \in \mathcal{F}$, and assume that a minimizer $f_{L_{0}}$ of $\|K(g-f)\|$ subject to $L f=L_{0}$ and $f \in \mathcal{F}$ exists for all $L_{0} \in \mathbb{R}$. Then the confidence set $\mathcal{C}_{g}$ that minimizes $E_{g} \lambda(\mathcal{C})$ subject to $\mathcal{C} \in \mathcal{I}_{\alpha}$ inverts the family of tests $\phi_{L_{0}}$ that reject for large values of $\left\langle K\left(g-f_{L_{0}}\right), Y\right\rangle$ with critical value given by the $1-\alpha$ quantile under $f_{L_{0}}$. Its expected length is

$$
E_{g}\left[\lambda\left(\mathcal{C}_{g}\right)\right]=(1-\alpha) E\left[\left(\omega\left(\sigma\left(z_{1-\alpha}-Z\right) ; \mathcal{F},\{g\}\right)+\omega\left(\sigma\left(z_{1-\alpha}-Z\right) ;\{g\}, \mathcal{F}\right)\right) \mid Z \leq z_{1-\alpha}\right]
$$

where $Z$ is a standard normal random variable.

This result solves the problem of "adaptation to a function" posed by Cai et al. (2013), who obtain bounds for this problem if $\mathcal{C}$ is required to be an interval. The theorem uses the observation in Pratt (1961) that minimum expected length CIs are obtained by inverting a family of uniformly most powerful tests of $H_{0}: L f=L_{0}$ and $f \in \mathcal{F}$ against $H_{1}: f=g$, which, as shown in the proof, is given by $\phi_{L_{0}}$; the expression for the expected length of $\mathcal{C}_{g}$ follows by computing the power of these tests. The assumption on the existence of the minimizer $f_{L_{0}}$ means that $L f$ is unbounded over $\mathcal{F}$, and it is made to simplify the statement; a truncated version of the same formula holds when $\mathcal{F}$ places a bound on $L f$.

Directing power at a single function is seldom desirable in practice. Theorem 3.2 is very useful, however, in bounding the efficiency of other procedures. In particular, suppose $f-g \in \mathcal{F}$ for all $f$, so that $(27)$ holds with $\mathcal{G}=\{g\}$ (such as when $g$ is the zero function), and

\footnotetext{
${ }^{6}$ We assume that $\omega^{\prime}(\delta ; \mathcal{F})=\operatorname{sd}\left(\hat{L}_{\delta, \mathcal{F}}\right) / \sigma \neq 0$. Otherwise, the estimator $\hat{L}_{\delta, \mathcal{F}}$ doesn't depend on the data, and the only valid fixed-length CI around it is the trivial CI that reports the whole parameter space for $L f$.
} 
that $\mathcal{F}$ is centrosymmetric. Then, by arguments in the proof of Corollary $3.2, \omega(\delta ; \mathcal{F},\{g\})=$ $\omega(\delta ;\{g\}, \mathcal{F})=\frac{1}{2} \omega(2 \delta ; \mathcal{F})$, which yields:

Corollary 3.3. Consider the setup in Theorem 3.2 with the additional assumption that $\mathcal{F}$ is centrosymmetric and $g$ satisfies $f-g \in \mathcal{F}$ for all $f$. Then the efficiency of the fixed-length $C I$ around $\hat{L}_{\delta_{\chi}, \mathcal{F}}$ at $g$ relative to all confidence sets is

$$
\frac{\inf _{\mathcal{C} \in \mathcal{I}_{\alpha}} E_{g} \lambda(\mathcal{C}(Y))}{2 \chi_{\alpha}\left(\hat{L}_{\delta_{\chi}, \mathcal{F}}\right)}=\frac{(1-\alpha) E\left[\omega\left(2 \sigma\left(z_{1-\alpha}-Z\right) ; \mathcal{F}\right) \mid Z \leq z_{1-\alpha}\right]}{2 \operatorname{cv}_{\alpha}\left(\frac{\omega\left(\delta_{\chi} ; \mathcal{F}\right)}{2 \sigma \omega^{\prime}\left(\delta_{\chi} ; \mathcal{F}\right)}-\frac{\delta_{\chi}}{2 \sigma}\right) \cdot \sigma \omega^{\prime}\left(\delta_{\chi} ; \mathcal{F}\right)}
$$

The efficiency ratio (30) can easily be computed in particular applications, and we do so in the empirical application in Section 4. When the one-class modulus satisfies (29), then, as in the case of one-sided CIs, the asymptotic efficiency of the fixed-length CI around $\hat{L}_{\delta_{\chi}}$ can be shown to depend only on $r$ and $\alpha$, and we plot it in Figure 2 for $\alpha=0.05$ (see Theorem E. 1 for the formula). When $r=1$ (parametric rate of convergence) and $\alpha=0.05$, the asymptotic efficiency equals $84.99 \%$, as in the normal mean example in Pratt (1961, Section 5).

Just like with minimax one-sided CIs, this result places a severe bound on the scope for improvement over fixed-length CIs when $\mathcal{F}$ is centrosymmetric. It strengthens the finding in Low (1997) and Cai and Low (2004a), who derive bounds on the expected length of random length $1-\alpha$ CIs. Their bounds imply that when $\mathcal{F}$ is constrained only by bounds on a derivative, the expected length of any CI in $\mathcal{I}_{\alpha}$ must shrink at the minimax rate $n^{-r / 2}$ for any $g$ in the interior of $\mathcal{F}{ }^{7}$ Figure 2 shows that for smooth functions $g$, this remains true whenever $\mathcal{F}$ is centrosymmetric, even if we don't require $\mathcal{C}$ to take the form of an interval. Importantly, the figure also shows that not only is the rate the same as the minimax rate, the constant must be close to that for fixed-length CIs. Since adapting to a single function $g$ is easier than adapting to any class $\mathcal{G}$ that includes it, this result effectively rules out adaptation to subclasses of $\mathcal{F}$ that contain smooth functions.

\section{Empirical illustration}

In this section, we illustrate the theoretical results in an RD application using a dataset from Lee (2008). The dataset contains 6,558 observations on elections to the US House

\footnotetext{
${ }^{7}$ One can use Theorem 3.2 to show that this result holds even if we don't require $\mathcal{C}$ to take the form of an interval. For example, in the RD model with $\mathcal{F}=\mathcal{F}_{R D T, p}(C)$ and $g \in \mathcal{F}_{R D T, p}\left(C_{g}\right), C_{g}<C$, the result follows from lower bounding $E_{g}\left[\lambda\left(\mathcal{C}_{g}\right)\right]$ using $\omega(\delta ; \mathcal{F},\{g\})+\omega(\delta ;\{g\}, \mathcal{F}) \geq \omega\left(2 \delta, \mathcal{F}_{R D T, p}\left(C-C_{g}\right)\right)$.
} 
of Representatives between 1946 and 1998. The running variable $x_{i} \in[-100,100]$ is the Democratic margin of victory (in percentages) in election $i$. The outcome variable $y_{i} \in$ $[0,100]$ is the Democratic vote share (in percentages) in the next election. Given the inherent uncertainty in final vote counts, the party that wins is essentially randomized in elections that are decided by a narrow margin, so that the RD parameter $L f$ measures the incumbency advantage for Democrats for elections decided by a narrow margin - the impact of being the current incumbent party in a congressional district on the vote share in the next election.

We consider inference under the Taylor class $\mathcal{F}_{R D T, p}(C)$, with $p=2$. We report results for the optimal estimators and CIs, as well as CIs based on local linear estimators, using the formulas described in Section 2.2 (which follow from the general results in Section 3). We use the preliminary estimates $\hat{\sigma}_{+}^{2}(x)=12.6^{2}$ and $\hat{\sigma}_{-}^{2}(x)=10.8^{2}$ in Step 1 , which are based on residuals form a local linear regression with bandwidth selected using the Imbens and Kalyanaraman (2012) selector. In Step 4, we use the nearest-neighbor variance estimator with $J=3$.

Let us briefly discuss the interpretation of the smoothness constant $C$ in this application. By definition of the class $\mathcal{F}_{R D T, 2}(C), C$ determines how large the approximation error can be if we approximate the regression functions $f_{+}$and $f_{-}$on either side of the cutoff by a linear Taylor approximation at the cutoff: the approximation error is no greater than $C x^{2}$. One way of gauging the magnitude of this approximation error is to look at its effect on prediction error when using the Taylor approximation to predict the vote share in the next election, and the margin in the previous election was $x_{0}$. If one uses the Taylor approximation, the prediction MSE is at most $C^{2} x_{0}^{4}+\sigma^{2}\left(x_{0}\right)$, whereas using the true conditional mean to predict the vote share would lead to prediction MSE $\sigma^{2}\left(x_{0}\right)$. Thus, using the true conditional mean leads to a MSE reduction in this prediction problem by a factor of at most $C^{2} x_{0}^{4} /\left(C^{2} x_{0}^{4}+\sigma^{2}\left(x_{0}\right)\right)$. If $C=0.05$ for instance, this implies MSE reductions of at most $13.6 \%$ at $x_{0}=10 \%$, and $71.5 \%$ at $x_{0}=20 \%$, assuming that $\sigma^{2}\left(x_{0}\right)$ equals our estimate of $12.6^{2}$. To the extent that researchers agree that the vote share in the next election varies smoothly enough with the margin of victory in the current election to make such large reductions in MSE unlikely, $C=0.05$ is quite a conservative choice.

Our adaptivity bounds imply that one cannot use data-driven methods to tighten our CIs, by say, estimating $C$. It is, however, possible to lower-bound the value of $C$. We derive a simple estimate of this lower bound in Supplemental Appendix E.3, which in the Lee data yields the lower bound estimate 0.017. As detailed in the appendix, the lower bound estimate can also be used in a model specification test to check whether a given chosen value of $C$ is 
too low. To examine sensitivity of the results to different choices of $C$, we present the results for the range $C \in[0.0002,0.1]$ that, by the argument in the preceding paragraph, includes most plausible values.

\subsection{Optimal and near-optimal confidence intervals}

The top panel in Figure 3 plots the optimal one- and two-sided CIs defined in Section 2, as well as estimates based on minimizing the worst-case MSE (see Remark 2.2). The estimates vary between $5.8 \%$ and $7.4 \%$ for $C \geq 0.005$, which is close to the original Lee estimate of $7.7 \%$ that was based on a global fourth degree polynomial. Interestingly, the lower and upper limits $\hat{c}_{u}$ and $\hat{c}_{\ell}$ of the one-sided CIs $\left[\hat{c}_{\ell}, \infty\right)$ and $\left(-\infty, \hat{c}_{u}\right]$ are not always within the corresponding limits for the two-sided CIs. The reason for this is that for any given $C$, the optimal smoothing parameters $h_{+}$and $h_{-}$are smaller for one-sided CIs than for two-sided fixed-length CIs. Thus, when the point estimate decreases with the amount of smoothing as is the case for low values of $C$, then one-sided CIs are effectively centered around a lower estimate, which explains why at first the one-sided CI limits are both below the two-sided limits. This reverses once the point estimate starts increasing with the amount of smoothing. Furthermore, the optimal smoothing parameters for the minimax MSE estimator are slightly smaller than those for fixed-length CIs throughout the entire range of $C \mathrm{~s}$, albeit by a small amount. This matches the asymptotic predictions in Armstrong and Kolesár (2016b).

As we discussed in Remark 2.2, it may be desirable to report an estimate with good MSE, with a CI centered at this estimate (without reoptimizing the smoothing parameters). The bottom panel in Figure 3 gives CIs with the smoothing parameters chosen so that the $\hat{L}_{h_{+}, h_{-}}$ minimizes the maximum MSE. The limits of the one-sided CIs are now contained within the two-sided CIs, as they are both based on the same estimator, although they are less

than $\left(z_{1-\alpha / 2}-z_{1-\alpha}\right) \operatorname{sd}\left(\hat{L}_{h_{+}, h_{-}}\right)$apart as would be the case if $\hat{L}_{h_{+}, h_{-}}$were unbiased. Finally, Figure 4 considers CIs based on local linear estimators with triangular kernel; these CIs are very close to the optimal CIs in Figure 3.

\subsection{Efficiency comparisons and bounds on adaptation}

We now consider the relative efficiency of the different CIs reported in Figures 3 and 4. To keep the efficiency comparisons meaningful, we assume that the variance is homoskedastic on either side of the cutoff, and equal to the initial estimates.

First, comparing half-length and excess length of CIs based on choosing $h_{+}, h_{-}$to min- 
imize the MSE to that of CIs based on optimally chosen $h_{+}$and $h_{-}$, we find that over the range of $C$ 's considered, for both optimal and local linear estimators, two-sided CIs based on MSE-optimal estimators are at least $99.9 \%$ efficient, and one-sided CIs are at least $97.7 \%$ efficient. These results are in line with the asymptotic results in Armstrong and Kolesár (2016b), which imply that the asymptotic efficiency of two-sided fixed-length CIs is $99.9 \%$, and it is $98.0 \%$ for one-sided CIs.

Second, comparing half-length and excess length of the CIs based on local linear estimates to that of CIs based on optimal estimators, we find that one- and two-sided CIs based on local linear estimators with triangular kernel are at least $96.9 \%$ efficient. This is very close to the asymptotic efficiency result in Armstrong and Kolesár (2016b) that the local linear estimator with a triangular kernel is $97.2 \%$ efficient, independently of the performance criterion.

Third, since the class $\mathcal{F}_{R D T, 2}(C)$ is centrosymmetric, we can use Corollaries 3.2 and 3.3 to bound the scope for adaptation to the class of piecewise linear functions, $\mathcal{G}=\mathcal{F}_{R D T, 2}(0)$. We find that the relative efficiency of CIs that minimax the 0.8 quantile is between $96 \%$ and $97.4 \%$, and the efficiency of fixed-length two-sided CIs at any $g \in \mathcal{G}$ is between $95.5 \%$ and $95.9 \%$ for the range of $C$ 's considered. This is very close to the asymptotic efficiency predictions, $96.7 \%$ and $95.7 \%$, respectively, implied by Figure 2 (with $r=4 / 5$ ). Thus, one cannot avoid choosing $C$ a priori. 


\section{Appendix A Proofs for main results}

This section contains proofs of the results in Section 3. Appendix A.1 contains auxiliary lemmas used in the proofs. The proofs of the results in Section 3 are given in the remainder of the section. Proofs of Corollaries 3.1 and 3.3 follow immediately from the theorems and arguments in the main text, and their proofs are omitted. We assume throughout this section that the sets $\mathcal{F}$ and $\mathcal{G}$ are convex.

Before proceeding, we recall that $\omega^{\prime}(\delta ; \mathcal{F}, \mathcal{G})$ was defined in Section 3 to be an arbitrary element of the superdifferential. We denote this set by

$$
\partial \omega(\delta ; \mathcal{F}, \mathcal{G})=\{d: \text { for all } \eta>0, \omega(\eta ; \mathcal{F}, \mathcal{G}) \leq \omega(\delta ; \mathcal{F}, \mathcal{G})+d(\eta-\delta)\}
$$

It is nonempty since $\omega(\cdot ; \mathcal{F}, \mathcal{G})$ is concave - if $f_{\delta}^{*}, g_{\delta}^{*}$ attain the modulus at $\delta$ and similarly for $\tilde{\delta}$, then, for $\lambda \in[0,1], f_{\lambda}=\lambda f_{\delta}^{*}+(1-\lambda) f_{\tilde{\delta}}^{*}$ and $g_{\lambda}=\lambda g_{\delta}^{*}+(1-\lambda) g_{\tilde{\delta}}^{*}$ satisfy $\left\|K\left(g_{\lambda}-f_{\lambda}\right)\right\| \leq$ $\lambda \delta+(1-\lambda) \tilde{\delta}$ so that $\omega(\lambda \delta+(1-\lambda) \tilde{\delta}) \geq L g_{\lambda}-L f_{\lambda}=\lambda \omega(\delta)+(1-\lambda) \omega(\tilde{\delta})$.

The definition of $\hat{L}_{\delta, \mathcal{F}, \mathcal{G}}$ in $(23)$ depends on the choice of $\omega^{\prime}(\delta ; \mathcal{F}, \mathcal{G}) \in \partial \omega(\delta ; \mathcal{F}, \mathcal{G})$ and $f_{\delta, \mathcal{F}, \mathcal{G}}^{*}, g_{\delta, \mathcal{F}, \mathcal{G}}^{*}$. As we explain in Supplemental Appendix D.2, Theorem 3.1 holds for any choice of $\omega^{\prime}(\delta ; \mathcal{F}, \mathcal{G})$ so long as the same element is used in the definition of the estimator and worst-case bias formula. Regarding the choice of the particular solution $f_{\delta, \mathcal{F}, \mathcal{G}}^{*}, g_{\delta, \mathcal{F}, \mathcal{G}}^{*}$ used to construct the estimator and CIs, it turns out that, under the conditions of Theorem 3.1, the choice does not affect the definition of $\hat{L}_{\delta, \mathcal{F}, \mathcal{G}}$ or the CIs based on it, as we now explain. If $\left(f_{0}^{*}, g_{0}^{*}\right)$ and $\left(f_{1}^{*}, g_{1}^{*}\right)$ solve the modulus problem with $K\left(g_{0}^{*}-f_{0}^{*}\right) \neq K\left(g_{1}^{*}-f_{1}^{*}\right)$, a strict convex combination $\left(f_{\lambda}, g_{\lambda}\right)$ will satisfy $\left\|K\left(f_{\lambda}-g_{\lambda}\right)\right\| \leq \delta-\eta$ for some $\eta>0$, which implies $\omega(\delta-\eta ; \mathcal{F}, \mathcal{G})=L\left(g_{\lambda}-f_{\lambda}\right)=\omega(\delta ; \mathcal{F}, \mathcal{G})$. Since the modulus is nondecreasing, this implies that it is constant in a neighborhood of $\delta$, so that $\partial \omega(\delta ; \mathcal{F}, \mathcal{G})=\{0\}$. Thus, either $K\left(g_{\delta}^{*}-f_{\delta}^{*}\right)$ is defined uniquely or $\partial \omega(\delta ; \mathcal{F}, \mathcal{G})=\{0\}$. In either case, $\omega^{\prime}(\delta ; \mathcal{F}, \mathcal{G}) \cdot K\left(f_{\delta}^{*}-g_{\delta}^{*}\right)$ is defined uniquely up to the choice of $\omega^{\prime}(\delta ; \mathcal{F}, \mathcal{G})$, which means that, for any two estimators $\hat{L}_{0}$ and $\hat{L}_{1}$ that satisfy the definition of $\hat{L}_{\delta, \mathcal{F}, \mathcal{G}}$ with the same choice of $\omega^{\prime}(\delta ; \mathcal{F}, \mathcal{G})$, we must have $\hat{L}_{1}=\hat{L}_{0}+a$ for some constant $a$. The bias formula (24), which follows from Lemma A.1 below, then implies that $a=0$. Similarly, the CIs $\left[\hat{c}_{\alpha, \mathcal{F}, \mathcal{G}}, \infty\right)$ and $\hat{L}_{\delta, \mathcal{F}, \mathcal{G}} \pm \chi_{\alpha}\left(\hat{L}_{\delta, \mathcal{F}, \mathcal{G}}\right)$ are defined uniquely up to the choice of $\omega^{\prime}(\delta ; \mathcal{F}, \mathcal{G})$. 


\section{A.1 Auxiliary lemmas}

The following lemma extends Lemma 4 in Donoho (1994) to the two class modulus (see also Theorem 2 in Cai and Low, 2004b, for a similar result in the Gaussian white noise model). The proof is essentially the same as for the single class case.

Lemma A.1. Let $\mathcal{F}$ and $\mathcal{G}$ be convex sets and let $f^{*}$ and $g^{*}$ solve the optimization problem for $\omega\left(\delta_{0} ; \mathcal{F}, \mathcal{G}\right)$ with $\left\|K\left(f^{*}-g^{*}\right)\right\|=\delta_{0}$, and let $d \in \partial \omega\left(\delta_{0} ; \mathcal{F}, \mathcal{G}\right)$. Then, for all $f \in \mathcal{F}$ and $g \in \mathcal{G}$,

$$
L g-L g^{*} \leq d \frac{\left\langle K\left(g^{*}-f^{*}\right), K\left(g-g^{*}\right)\right\rangle}{\left\|K\left(g^{*}-f^{*}\right)\right\|} \text { and } L f-L f^{*} \geq d \frac{\left\langle K\left(g^{*}-f^{*}\right), K\left(f-f^{*}\right)\right\rangle}{\left\|K\left(g^{*}-f^{*}\right)\right\|} .
$$

In particular, $\hat{L}_{\delta, \mathcal{F}, \mathcal{G}}$ achieves maximum bias over $\mathcal{F}$ at $f^{*}$ and minimum bias over $\mathcal{G}$ at $g^{*}$.

Proof. Denote the ordered modulus $\omega(\delta ; \mathcal{F}, \mathcal{G})$ by $\omega(\delta)$. Suppose that the first inequality in (31) does not hold for some $g$. Then, for some $\varepsilon>0$,

$$
L g-L g^{*}>(d+\varepsilon) \frac{\left\langle K\left(g^{*}-f^{*}\right), K\left(g-g^{*}\right)\right\rangle}{\left\|K\left(g^{*}-f^{*}\right)\right\|} .
$$

Let $g_{\lambda}=(1-\lambda) g^{*}+\lambda g$. Since $g_{\lambda}-g^{*}=\lambda\left(g-g^{*}\right)$, we have $\lambda L\left(g-g^{*}\right)=L g_{\lambda}-L f^{*}-$ $L\left(g^{*}-f^{*}\right)=L g_{\lambda}-L f^{*}-\omega\left(\delta_{0}\right)$. Furthermore, since $g_{\lambda} \in \mathcal{G}$ by convexity, $L g_{\lambda}-L f^{*} \leq$ $\omega\left(\left\|K\left(g_{\lambda}-f^{*}\right)\right\|\right)$ so multiplying $(32)$ by $\lambda$ gives

$$
\omega\left(\left\|K\left(g_{\lambda}-f^{*}\right)\right\|\right)-\omega\left(\delta_{0}\right) \geq \lambda L\left(g-g^{*}\right)>\lambda(d+\varepsilon) \frac{\left\langle K\left(g^{*}-f^{*}\right), K\left(g-g^{*}\right)\right\rangle}{\left\|K\left(g^{*}-f^{*}\right)\right\|} .
$$

Note that

$$
\left.\frac{d}{d \lambda}\left\|K\left(g_{\lambda}-f^{*}\right)\right\|\right|_{\lambda=0}=\frac{1}{2} \frac{\left.\frac{d}{d \lambda}\left\|K\left(g_{\lambda}-f^{*}\right)\right\|^{2}\right|_{\lambda=0}}{\left\|K\left(g^{*}-f^{*}\right)\right\|}=\frac{\left\langle K\left(g^{*}-f^{*}\right), K\left(g-g^{*}\right)\right\rangle}{\left\|K\left(g^{*}-f^{*}\right)\right\|}
$$

so that $\left\|K\left(g_{\lambda}-f^{*}\right)\right\|=\delta_{0}+\lambda \frac{\left\langle K\left(g^{*}-f^{*}\right), K\left(g-g^{*}\right)\right\rangle}{\left\|K\left(g^{*}-f^{*}\right)\right\|}+o(\lambda)$. Combining this with (33), we have

$$
\omega\left(\delta_{0}+\lambda \frac{\left\langle K\left(g^{*}-f^{*}\right), K\left(g-g^{*}\right)\right\rangle}{\left\|K\left(g^{*}-f^{*}\right)\right\|}+o(\lambda)\right)-\omega\left(\delta_{0}\right)>\lambda(d+\varepsilon) \frac{\left\langle K\left(g^{*}-f^{*}\right), K\left(g-g^{*}\right)\right\rangle}{\left\|K\left(g^{*}-f^{*}\right)\right\|},
$$

which is a contradiction unless $\left\langle K\left(g^{*}-f^{*}\right), K\left(g-g^{*}\right)\right\rangle=0$.

If $\left\langle K\left(g^{*}-f^{*}\right), K\left(g-g^{*}\right)\right\rangle=0$, then (32) gives $L g-L g^{*}>0$, which, by the first 
inequality in (33) implies $\omega\left(\left\|K\left(g_{\lambda}-f^{*}\right)\right\|\right)-\omega\left(\delta_{0}\right) \geq \lambda c$ where $c=L g-L g^{*}>0$. But in this case (34) implies $\left\|K\left(g_{\lambda}-f^{*}\right)\right\|=\delta_{0}+o(\lambda)$, again giving a contradiction. This proves the first inequality, and a symmetric argument applies to the inequality involving $L f-L f^{*}$, thereby giving the first result.

Now consider the test statistic $\hat{L}_{\delta, \mathcal{F}, \mathcal{G}}$. Under $g \in \mathcal{G}$, the bias of this statistic is equal to a constant that does not depend on $g$ plus

$$
d \frac{\left\langle K\left(g^{*}-f^{*}\right), K\left(g-g^{*}\right)\right\rangle}{\left\|K\left(g^{*}-f^{*}\right)\right\|}-\left(L g-L g^{*}\right) .
$$

It follows from (31) that this is minimized over $g \in \mathcal{G}$ by taking $g=g^{*}$. Similarly, the maximum bias over $\mathcal{F}$ is taken at $f^{*}$.

The next lemma is used in the proof of Theorem 3.2 .

Lemma A.2. Let $\tilde{\mathcal{F}}$ and $\tilde{\mathcal{G}}$ be convex sets, and suppose that $f^{*}$ and $g^{*}$ minimize $\|K(f-g)\|$ over $f \in \tilde{\mathcal{F}}$ and $g \in \tilde{\mathcal{G}}$. Then, for any level $\alpha$, the minimax test of $H_{0}: \tilde{\mathcal{F}}$ vs $H_{1}: \tilde{\mathcal{G}}$ is given by the Neyman-Pearson test of $f^{*}$ vs $g^{*}$. It rejects when $\left\langle K\left(f^{*}-g^{*}\right), Y\right\rangle$ is greater than its $1-\alpha$ quantile under $f^{*}$. The minimum power of this test over $\tilde{\mathcal{G}}$ is taken at $g^{*}$.

Proof. The result is immediate from results stated in Section 2.4.3 in Ingster and Suslina (2003), since the sets $\{K f: f \in \tilde{\mathcal{F}}\}$ and $\{K g: g \in \tilde{\mathcal{G}}\}$ are convex.

\section{A.2 Proof of Theorem 3.1}

For ease of notation in this proof, let $f^{*}=f_{\delta}^{*}$ and $g^{*}=g_{\delta}^{*}$ denote the functions that solve the modulus problem with $\left\|K\left(f^{*}-g^{*}\right)\right\|=\delta$, and let $d=\omega^{\prime}(\delta ; \mathcal{F}, \mathcal{G}) \in \partial \omega(\delta ; \mathcal{F}, \mathcal{G})$ so that, plugging the worst-case bias formula (24) into the definition of $\hat{c}_{\alpha}$, we have

$$
\hat{c}_{\alpha}=\hat{c}_{\alpha, \delta, \mathcal{F}, \mathcal{G}}=L f^{*}+d \frac{\left\langle K\left(g^{*}-f^{*}\right), Y\right\rangle}{\left\|K\left(g^{*}-f^{*}\right)\right\|}-d \frac{\left\langle K\left(g^{*}-f^{*}\right), K f^{*}\right\rangle}{\left\|K\left(g^{*}-f^{*}\right)\right\|}-z_{1-\alpha} \sigma d .
$$

Note that $\hat{c}_{\alpha}=\hat{L}_{\delta, \mathcal{F}, \mathcal{G}}+a$ for $a$ chosen so that the $1-\alpha$ quantile of $\hat{c}_{\alpha}-L f^{*}$ under $f^{*}$ is zero. Thus, it follows from Lemma A.1 that $\left[\hat{c}_{\alpha}, \infty\right)$ is a valid $1-\alpha$ CI for $L f$ over $\mathcal{F}$, and that all quantiles of excess coverage $L g-\hat{c}_{\alpha}$ are maximized over $\mathcal{G}$ at $g^{*}$. In particular, $q_{\beta}\left(\hat{c}_{\alpha} ; \mathcal{G}\right)=q_{g^{*}, \beta}\left(L g^{*}-\hat{c}_{\alpha}\right)$. To calculate this quantile, note that, under $g^{*}, L g^{*}-\hat{c}_{\alpha}$ is normal with variance $d^{2} \sigma^{2}$ and mean

$$
L g^{*}-L f^{*}-d \frac{\left\langle K\left(g^{*}-f^{*}\right), K\left(g^{*}-f^{*}\right)\right\rangle}{\left\|K\left(g^{*}-f^{*}\right)\right\|}+z_{1-\alpha} \sigma d=\omega(\delta ; \mathcal{F}, \mathcal{G})+d\left(z_{1-\alpha} \sigma-\delta\right) .
$$


The probability that this normal variable is less than or equal to $\omega(\delta ; \mathcal{F}, \mathcal{G})$ is given by the probability that a normal variable with mean $d\left(z_{1-\alpha} \sigma-\delta\right)$ and variance $d^{2} \sigma^{2}$ is less than or equal to zero, which is $\Phi\left(\delta / \sigma-z_{1-\alpha}\right)=\beta$. Thus, $q_{\beta}\left(\hat{c}_{\alpha} ; \mathcal{G}\right)=\omega(\delta ; \mathcal{F}, \mathcal{G})$ as claimed.

It remains to show that no other $1-\alpha$ CI can strictly improve on this. Suppose that some other $1-\alpha$ CI $[\tilde{c}, \infty)$ obtained $q_{\beta}(\tilde{c} ; \mathcal{G})<q_{\beta}\left(\hat{c}_{\alpha} ; \mathcal{G}\right)=\omega(\delta ; \mathcal{F}, \mathcal{G})$. Then the $\beta$ quantile of excess length at $g^{*}$ would be strictly less than $\omega(\delta ; \mathcal{F}, \mathcal{G})$, so that, for some $\eta>0$,

$$
P_{g^{*}}\left(L g^{*}-\tilde{c} \leq \omega(\delta ; \mathcal{F}, \mathcal{G})-\eta\right) \geq \beta
$$

Let $\tilde{f}$ be given by a convex combination between $g^{*}$ and $f^{*}$ such that $L g^{*}-L \tilde{f}=\omega(\delta ; \mathcal{F} ; \mathcal{G})-$ $\eta / 2$. Then the above display gives

$$
P_{g^{*}}(\tilde{c}>L \tilde{f}) \geq P_{g^{*}}(\tilde{c} \geq L \tilde{f}+\eta / 2)=P_{g^{*}}\left(L g^{*}-\tilde{c} \leq L g^{*}-L \tilde{f}-\eta / 2\right) \geq \beta .
$$

But this would imply that the test that rejects when $\tilde{c}>L \tilde{f}$ is level $\alpha$ for $H_{0}: \tilde{f}$ and has power $\beta$ at $g^{*}$. This can be seen to be impossible by calculating the power of the NeymanPearson test of $\tilde{f}$ vs $g^{*}$, since $\beta$ is the power of the Neyman-Pearson test of $f^{*}$ vs $g^{*}$, and $\tilde{f}$ is a strict convex combination of these functions.

\section{A.3 Proof of Corollary 3.2}

Under (27), if $f_{\delta, \mathcal{F}, \mathcal{G}}^{*}$ and $g_{\delta, \mathcal{F}, \mathcal{G}}^{*}$ solve the modulus problem $\omega(\delta, \mathcal{F}, \mathcal{G})$, then $f_{\delta, \mathcal{F}, \mathcal{G}}^{*}-g_{\delta, \mathcal{F}, \mathcal{G}}^{*}$ and 0 (the zero function) solve $\omega(\delta ; \mathcal{F},\{0\})$. Thus, $\omega(\delta ; \mathcal{F}, \mathcal{G})=\omega(\delta ; \mathcal{F},\{0\})$, and the estimators $\hat{L}_{\delta, \mathcal{F}, \mathcal{G}}$ and $\hat{L}_{\delta, \mathcal{F},\{0\}}$ and the corresponding CIs are equal up to the choice of the element in the superdifferential. It therefore suffices to prove the result for $\mathcal{G}=\{0\}$.

We have

$$
\omega(\delta ; \mathcal{F},\{0\})=\sup \{-L f:\|K f\| \leq \delta, f \in \mathcal{F}\}=\frac{1}{2} \omega(2 \delta ; \mathcal{F}),
$$

where the last equality obtains because under centrosymmetry, maximizing $-L f=L(-f)$ and maximizing $L f$ are equivalent, so that the maximization problem is equivalent to (25). Furthermore, we can take $g_{2 \delta, \mathcal{F}}^{*}, f_{2 \delta, \mathcal{F}}^{*}$ to satisfy $g_{2 \delta, \mathcal{F}}^{*}=-f_{2 \delta, \mathcal{F}}^{*}$ with $f_{2 \delta, \mathcal{F}}^{*}$ solving the above optimization problem, so that $g_{\delta, \mathcal{F},\{0\}}^{*}-f_{\delta, \mathcal{F},\{0\}}^{*}=-f_{\delta, \mathcal{F},\{0\}}^{*}=-f_{2 \delta, \mathcal{F}}^{*}=\frac{1}{2}\left(g_{2 \delta, \mathcal{F}}^{*}-f_{2 \delta, \mathcal{F}}^{*}\right)$. Thus, $\hat{L}_{\delta, \mathcal{F},\{0\}}$ and $\hat{L}_{2 \delta, \mathcal{F}}$ are equal up to a constant, which implies $\hat{c}_{\alpha, \delta, \mathcal{F},\{0\}}=\hat{c}_{\alpha, 2 \delta, \mathcal{F}}$. This proves the first part of the corollary. The second part of the corollary follows by plugging bias $_{\{0\}}\left(\hat{L}_{\delta_{\beta}, \mathcal{F}}\right)=0$ and the formulas for $\overline{\operatorname{bias}}_{\mathcal{F}}\left(\hat{L}_{\delta_{\beta}, \mathcal{F}}\right)$ and $\operatorname{sd}\left(\hat{L}_{\delta_{\beta}, \mathcal{F}}\right)$ given in Section 3.2 into the expression $(21)$ to obtain $q_{\beta}\left(\hat{c}_{\alpha, \delta_{\beta}, \mathcal{F}},\{0\}\right)=\left(\omega\left(\delta_{\beta} ; \mathcal{F}\right)+\delta_{\beta} \omega^{\prime}\left(\delta_{\beta} ; \mathcal{F}\right)\right) / 2$. 


\section{A.4 Proof of Theorem 3.2}

Following Pratt (1961), note that, for any confidence set $\mathcal{C}$ for $\vartheta=L f$, we have

$$
E_{g} \lambda(\mathcal{C})=E_{g} \int\left(1-\phi_{\mathcal{C}}(\vartheta)\right) d \vartheta=\int E_{g}\left(1-\phi_{\mathcal{C}}(\vartheta)\right) d \vartheta
$$

by Fubini's theorem, where $\phi_{\mathcal{C}}(\vartheta)=1(\vartheta \notin \mathcal{C})$. Thus, the CI that minimizes this inverts the family of most powerful tests of $H_{0}: L f=\vartheta, f \in \mathcal{F}$ against $H_{1}: f=g$. By Lemma A.2 since the sets $\{f: L f=\vartheta, f \in \mathcal{F}\}$ and $\{g\}$ are convex, the least favorable function $f_{\vartheta}$ minimize $\|K(g-f)\|$ subject to $L f=\vartheta$, which gives the first part of the theorem.

To derive the expression for expected length, note that if $L g \leq \vartheta$, then the minimization problem is equivalent to solving the inverse ordered modulus problem $\omega^{-1}(\vartheta-L g ;\{g\}, \mathcal{F})$, and if $L g \geq \vartheta$, it is equivalent to solving $\omega^{-1}(L g-\vartheta ; \mathcal{F},\{g\})$. This follows because if the ordered modulus $\omega(\delta ; \mathcal{F},\{g\})$ is attained at some $f_{\delta}^{*}$ and $g$, then the inequality $\|K(f-g)\| \leq$ $\delta$ must be binding: otherwise a convex combination of $\tilde{f}$ and $f_{\delta}^{*}$, where $\tilde{f}$ is such that $L\left(g-f_{\delta}^{*}\right)<L(g-\tilde{f})$ would achieve a strictly larger value, and similarly for $\omega(\delta ;\{g\}, \mathcal{F})$. Such $\tilde{f}$ always exists since by the assumption that $f_{\vartheta}$ exists for all $\vartheta$. The above argument assumes that $\vartheta-L g \geq \omega(0 ;\{g\}, \mathcal{F})$ so that $\vartheta-L g$ is in the range of the modulus; if $0 \leq \vartheta-L g \leq \omega(0 ;\{g\}, \mathcal{F})$, then $\left\|K\left(f_{\vartheta}-g\right)\right\|=0$ so the minimization problem is still equivalent to the inverse modulus if we define the inverse to be 0 in this case (and similarly for $0 \leq L g-\vartheta \leq \omega(0 ; \mathcal{F},\{g\}))$.

Next, it follows from the proof of Theorem 3.1 that the power of the test $\phi_{\vartheta}$ at $g$ is given by $\Phi\left(\delta_{\vartheta} / \sigma-z_{1-\alpha}\right)$, where $\delta_{\vartheta}=\left\|f_{\vartheta}-g\right\|$. Therefore,

$$
E_{g}\left[\lambda\left(\mathcal{C}_{g}(Y)\right)\right]=\int \Phi\left(z_{1-\alpha}-\frac{\delta_{\vartheta}}{\sigma}\right) \mathrm{d} \vartheta=\iint 1\left(\delta_{\vartheta} \leq \sigma\left(z_{1-\alpha}-z\right)\right) \mathrm{d} \vartheta \mathrm{d} \Phi(z),
$$

where the second equality swaps the order of integration. Splitting the inner integral, using fact that $\delta_{\vartheta}=\omega^{-1}(L g-\vartheta ; \mathcal{F},\{g\})$ for $\vartheta \leq L g$ and $\delta_{\vartheta}=\omega^{-1}(\vartheta-L g ;\{g\}, \mathcal{F})$ for $\vartheta \geq L g$, and taking a modulus on both sides of the inequality of the integrand then yields

$$
\begin{aligned}
E_{g}\left[\lambda\left(\mathcal{C}_{g}(Y)\right)\right]= & \iint_{\vartheta \leq L g} 1\left(L g-\vartheta \leq \omega\left(\sigma\left(z_{1-\alpha}-z\right) ; \mathcal{F},\{g\}\right)\right) 1\left(z \leq z_{1-\alpha}\right) \mathrm{d} \vartheta \mathrm{d} \Phi(z) \\
& \quad+\iint_{\vartheta>L g} 1\left(\vartheta-L g \leq \omega\left(\sigma\left(z_{1-\alpha}-z\right) ;\{g\}, \mathcal{F}\right)\right) 1\left(z \leq z_{1-\alpha}\right) \mathrm{d} \vartheta \mathrm{d} \Phi(z) \\
= & (1-\alpha) E\left[\left(\omega\left(\sigma\left(z_{1-\alpha}-Z\right) ; \mathcal{F},\{g\}\right)+\omega\left(\sigma\left(z_{1-\alpha}-Z\right) ;\{g\}, \mathcal{F}\right)\right) \mid Z \leq z_{1-\alpha}\right],
\end{aligned}
$$


where $Z$ is standard normal, which yields the result.

\section{Appendix B Extension to RD with covariates}

This section discusses extensions to the RD setup when we have available a set of covariates $z_{i}$ that are independent of the treatment. If the object of interest is still the average treatment effect at $x=0$, then ignoring the additional covariates will still lead to a valid CI. However, one may want to use the information that $z_{i}$ is independent of treatment to gain precision. We discuss this in Appendix B.1. Alternatively, one may want to estimate the treatment effect at $x=0$ conditional on different values of $z$, which leads to a different approach, discussed in Appendix B.2.

\section{B.1 Using covariates to improve precision}

As argued by Calonico et al. (2017), if $z_{i}$ is independent of treatment, the conditional mean of $z_{i}$ given the running variable $x_{i}$ should be smooth near the cutoff. We can fit this into our setup using the model

$$
\begin{aligned}
& y_{i}=h_{y}\left(x_{i}\right)+u_{i}, \\
& z_{i}=h_{z}\left(x_{i}\right)+v_{i},
\end{aligned} \quad\left(\begin{array}{l}
u_{i} \\
v_{i}
\end{array}\right) \sim \mathcal{N}\left(0, \Sigma\left(x_{i}\right)\right), h_{y} \in \mathcal{H}_{y}, h_{z} \in \mathcal{H}_{z},
$$

where $\mathcal{H}_{y}$ and $\mathcal{H}_{z}$ are convex smoothness classes, and we treat $\Sigma(\cdot)$ as known. We incorporate the constraint that $z_{i}$ is independent of treatment by choosing a class $\mathcal{H}_{z}$ such that $\lim _{x \downarrow 0} h_{z}(x)-\lim _{x \uparrow 0} h_{z}(x)=0$ for all $h_{z} \in \mathcal{H}_{z}$. For example, we can take $\mathcal{H}_{y}=\mathcal{F}_{R D T, p}\left(C_{y}\right)$ and $\mathcal{H}_{z}=\mathcal{F}_{R D T, p}\left(C_{z}\right) \cap\left\{h: \lim _{x \downarrow 0} h_{z}(x)-\lim _{x \uparrow 0} h_{z}(x)=0\right\}$ for some constants $C_{y}$ and $C_{z}$.

Using our general results, one can compute optimal CIs and bounds for adaptation. For example, our adaptation bounds show that, when $\mathcal{H}_{y}$ and $\mathcal{H}_{z}$ are centrosymmetric, there are severe limitations to adapting to the smoothness constant for either class. Thus, CIs that take into account the covariates $z_{i}$ will have to depend explicitly on the smoothness constant that $h_{z}$ is assumed to satisfy.

In the remainder of this section, we consider a particular smoothness class, and we construct CIs that are optimal or near-optimal when $\Sigma(x)$ is constant as well as feasible versions of these CIs that are valid when $\Sigma(x)$ is unknown and may not be constant. Given $\Sigma$, let $\Sigma_{22}$ denote the bottom-right $d_{z} \times d_{z}$ submatrix of $\Sigma$ and let $\Sigma_{21}$ denote the bottom-left 
$d_{z} \times d_{1}$ submatrix of $\Sigma$, where $d_{z}$ is the dimension of $z_{i}$. Let $\tilde{y}_{i}=y_{i}-z_{i}^{\prime} \Sigma_{22}^{-1} \Sigma_{21}$ so that

$$
\tilde{y}_{i}=h_{y}\left(x_{i}\right)-h_{z}\left(y_{i}\right)^{\prime} \Sigma_{22}^{-1} \Sigma_{21}+u_{i}-v_{i}^{\prime} \Sigma_{22}^{-1} \Sigma_{21}=\tilde{h}_{y}\left(x_{i}\right)+\tilde{u}_{i}
$$

where $\tilde{h}_{y}\left(x_{i}\right)=h_{y}\left(x_{i}\right)-h_{z}\left(y_{i}\right)^{\prime} \Sigma_{22}^{-1} \Sigma_{21}$ and $\tilde{u}_{i}=u_{i}-v_{i}^{\prime} \Sigma_{22}^{-1} \Sigma_{21}$. Note also that $\lim _{x \downarrow 0} \tilde{h}_{y}(x)-$ $\lim _{x \uparrow 0} \tilde{h}_{y}(x)=\lim _{x \downarrow 0} h_{y}(x)-\lim _{x \uparrow 0} h_{y}(x)$, so that the RD parameter for $\tilde{h}_{y}$ is the same as the RD parameter for $h_{y}$. Suppose that we model the smoothness of $\tilde{h}_{y}$ directly, and take the parameter space for $\left(\tilde{h}_{y}, h_{z}\right)$ to be $\mathcal{F}_{R D T, p}(\tilde{C}) \times \mathcal{H}_{z}$. Since $\tilde{u}_{i}$ is independent of $v_{i}$ and the RD parameter depends only on $\tilde{h}_{y}$, it can be seen that minimax optimal estimators and CIs can be formed by ignoring the $z_{i}$ 's after this transformation is made. Thus, one can proceed as in Section 2.2 with $\tilde{y}_{i}$ in place of $y_{i}{ }^{8}$

To make this procedure feasible, we need an estimate of $\Sigma_{22}^{-1} \Sigma_{21}$. We propose the estimates $\hat{\Sigma}_{22}=\frac{1}{n h} \sum_{i=1}^{n} \hat{v}_{i} \hat{v}_{i}^{\prime} k\left(x_{i} / h\right)$ and $\hat{\Sigma}_{21}=\frac{1}{n h} \sum_{i=1}^{n} \hat{v}_{i} y_{i} k\left(x_{i} / h\right)$ where $\hat{v}_{i}$ is the residual from the local polynomial regression of $z_{i}$ on a $p$ th order polynomial of $x_{i}$ and its interaction with $1\left(x_{i}>0\right)$, with weight $k\left(x_{i} / h\right)$. To form CIs, one proceeds as in Section 2.2 with $\tilde{y}_{i}=y_{i}-z_{i}^{\prime} \hat{\Sigma}_{22}^{-1} \hat{\Sigma}_{21}$ in place of $y_{i}$ and $\tilde{C}$ playing the role of $C$. A simple calculation shows that, if one uses the local polynomial weights (14), with the same kernel and bandwidth used to estimate $\Sigma$, the resulting CIs will be centered at a local polynomial estimate where $z_{i}$ is included as a regressor in the local polynomial regression. This corresponds exactly to an estimator proposed by Calonico et al. (2017). Thus, our relative efficiency results can be used to show that this estimator is close to optimal under these assumptions.

\section{B.2 Estimating the treatment effect conditional on $z_{i}=z$}

If one is interested in how the treatment effect at $x=0$ varies with $z$, one can use the model $y_{i}=f\left(x_{i}, z_{i}\right)+u_{i}$ where $f$ is placed in a smoothness class and the object of interest is $L_{z} f=\lim _{x \downarrow 0} f(x, z)-\lim _{x \uparrow 0} f(x, z)$ for different values of $z$. This fits into our general framework once one fixes the point $z$ at which $L_{z} f$ is evaluated, and one can use our results to obtain CIs for different values of $z$. A natural smoothness class is to place a bound on the $p$ th order multivariate Taylor approximation of $f(x, z) 1(x>0)$ and $f(x, z) 1(x<0)$ at $x=0$ and $z$ equal to the value of interest. The analysis of optimal and near optimal estimators

\footnotetext{
${ }^{8}$ If one places smoothness assumptions on $h_{y}$ rather than $\tilde{h}_{y}$ by taking $\mathcal{H}_{y}=\mathcal{F}_{R D T, p}\left(C_{y}\right)$ and $\mathcal{H}_{z}=$ $\mathcal{F}_{R D T, p}\left(C_{z}\right) \cap\left\{h: \lim _{x \downarrow 0} h_{z}(x)-\lim _{x \uparrow 0} h_{z}(x)=0\right\}$, then $\tilde{h}_{y} \in \mathcal{F}_{R D T, p}\left(C_{y}+C_{z} \iota^{\prime} \Sigma_{22}^{-1} \Sigma_{21}\right)$ where $\iota$ is a vector of ones. It follows that the CIs discussed here will be valid for $\tilde{C} \geq C_{y}+C_{z} \iota^{\prime} \Sigma_{22}^{-1} \Sigma_{21}$. However, the resulting parameter space for $\left(\tilde{h}_{y}, h_{z}\right)$ will be different (in particular, it will not take the form $\mathcal{H}_{y} \times \mathcal{H}_{z}$ ), so that optimal estimators will be different for this class.
} 
then follows from a generalization of the results described in Section 2.2. In particular, one can use multivariate local polynomial estimators (with worst-case bias computed using a generalization of the calculations in Supplemental Appendix E.1), or optimal weights can be computed by generalizing the calculations in Supplemental Appendix E.2.

Estimating the treatment effect conditional on different values of $z$ can be a useful way of exploring treatment effect heterogeneity. However, unless one places some additional parametric structure on $f(x, z)$, the resulting estimates will suffer from imprecision when the dimension of $z$ is moderate due to the curse of dimensionality. 


\section{References}

Abadie, A. And G. W. Imbens (2006): "Large sample properties of matching estimators for average treatment effects," Econometrica, 74, 235-267.

Andrews, D. W. K. And P. Guggenberger (2009): "Hybrid and Size-Corrected Subsampling Methods," Econometrica, 77, 721-762.

Armstrong, T. B. (2015): "Adaptive testing on a regression function at a point," The Annals of Statistics, 43, 2086-2101.

Armstrong, T. B. And M. Kolesár (2016a): "Optimal inference in a class of regression models," ArXiv:1511.06028v2, https://arxiv.org/abs/1511.06028v2.

(2016b): "Simple and honest confidence intervals in nonparametric regression," ArXiv:1606.01200, https://arxiv.org/abs/1606.01200.

Belloni, A., V. Chernozhukov, and C. Hansen (2014): "Inference on Treatment Effects after Selection among High-Dimensional Controls," The Review of Economic Studies, 81, 608-650.

CaI, T. T. And M. G. Low (2004a): "An adaptation theory for nonparametric confidence intervals," Annals of Statistics, 32, 1805-1840.

- (2004b): "Minimax estimation of linear functionals over nonconvex parameter spaces," Annals of Statistics, 32, 552-576.

CAI, T. T., M. G. Low, AND Z. MA (2014): "Adaptive Confidence Bands for Nonparametric Regression Functions," Journal of the American Statistical Association, 109, 1054-1070.

CaI, T. T., M. G. Low, And Y. Xia (2013): "Adaptive confidence intervals for regression functions under shape constraints," The Annals of Statistics, 41, 722-750.

Calonico, S., M. D. Cattaneo, M. H. Farrell, and R. Titiunik (2017): "Regression Discontinuity Designs Using Covariates," Unpublished Manuscript, University of Michigan.

Calonico, S., M. D. Cattaneo, and R. Titiunik (2014): "Robust Nonparametric Confidence Intervals for Regression-Discontinuity Designs," Econometrica, 82, 2295-2326. 
Cheng, M.-Y., J. FAn, And J. S. Marron (1997): "On automatic boundary corrections," The Annals of Statistics, 25, 1691-1708.

Chernozhukov, V., D. Chetverikov, and K. Kato (2014): "Anti-concentration and honest, adaptive confidence bands," The Annals of Statistics, 42, 1787-1818.

Cohen, J. (1988): Statistical Power Analysis for the Behavioral Sciences, Hillsdale, NJ: Lawrence Erlbaum Associates.

Donoho, D. L. (1994): "Statistical Estimation and Optimal Recovery," The Annals of Statistics, 22, 238-270.

Donoho, D. L. And R. C. Liu (1991): "Geometrizing Rates of Convergence, III," The Annals of Statistics, 19, 668-701.

Donoho, D. L. And M. G. Low (1992): "Renormalization Exponents and Optimal Pointwise Rates of Convergence," The Annals of Statistics, 20, 944-970.

FAn, J. (1993): "Local Linear Regression Smoothers and Their Minimax Efficiencies," The Annals of Statistics, 21, 196-216.

Giné, E. AND R. NiCKL (2010): "Confidence bands in density estimation," The Annals of Statistics, 38, 1122-1170.

Hall, P. And J. Horowitz (2013): "A simple bootstrap method for constructing nonparametric confidence bands for functions," The Annals of Statistics, 41, 1892-1921.

Ibragimov, I. A. And R. Z. Khas'Minskil (1985): "On Nonparametric Estimation of the Value of a Linear Functional in Gaussian White Noise," Theory of Probability \&3 Its Applications, 29, 18-32.

Imbens, G. W. and K. Kalyanaraman (2012): "Optimal bandwidth choice for the regression discontinuity estimator," The Review of Economic Studies, 79, 933-959.

Ingster, Y. I. AND I. A. Suslina (2003): Nonparametric goodness-of-fit testing under Gaussian models, New York: Springer.

LEE, D. S. (2008): "Randomized experiments from non-random selection in U.S. House elections," Journal of Econometrics, 142, 675-697. 
LEE, D. S. AND D. CARD (2008): "Regression discontinuity inference with specification error," Journal of Econometrics, 142, 655-674.

Lehmann, E. L. And J. P. Romano (2005): Testing statistical hypotheses, New York: Springer, third ed.

Low, M. G. (1997): "On nonparametric confidence intervals," The Annals of Statistics, 25, $2547-2554$.

McCloskey, A. (2017): "Bonferroni-Based Size-Correction for Nonstandard Testing Problems," Journal of Econometrics, 200, 17-35.

PRATT, J. W. (1961): "Length of confidence intervals," Journal of the American Statistical Association, 56, 549-567.

SACKS, J. AND D. Ylvisaker (1978): "Linear Estimation for Approximately Linear Models," The Annals of Statistics, 6, 1122-1137.

Stone, C. J. (1980): "Optimal Rates of Convergence for Nonparametric Estimators," The Annals of Statistics, 8, 1348-1360. 


\begin{tabular}{llll} 
& \multicolumn{3}{c}{$\alpha$} \\
\cline { 2 - 4 }$b$ & 0.01 & 0.05 & 0.1 \\
\hline 0.0 & 2.576 & 1.960 & 1.645 \\
0.1 & 2.589 & 1.970 & 1.653 \\
0.2 & 2.626 & 1.999 & 1.677 \\
0.3 & 2.683 & 2.045 & 1.717 \\
0.4 & 2.757 & 2.107 & 1.772 \\
0.5 & 2.842 & 2.181 & 1.839 \\
0.6 & 2.934 & 2.265 & 1.916 \\
0.7 & 3.030 & 2.356 & 2.001 \\
0.8 & 3.128 & 2.450 & 2.093 \\
0.9 & 3.227 & 2.548 & 2.187 \\
1.0 & 3.327 & 2.646 & 2.284 \\
1.5 & 3.826 & 3.145 & 2.782 \\
2.0 & 4.326 & 3.645 & 3.282
\end{tabular}

Table 1: Critical values $\mathrm{cv}_{\alpha}(b)$ for selected confidence levels and values of maximum absolute bias $b$. For $b \geq 2, \mathrm{cv}_{\alpha}(b) \approx b+z_{1-\alpha}$ up to 3 decimal places for these values of $\alpha$. 


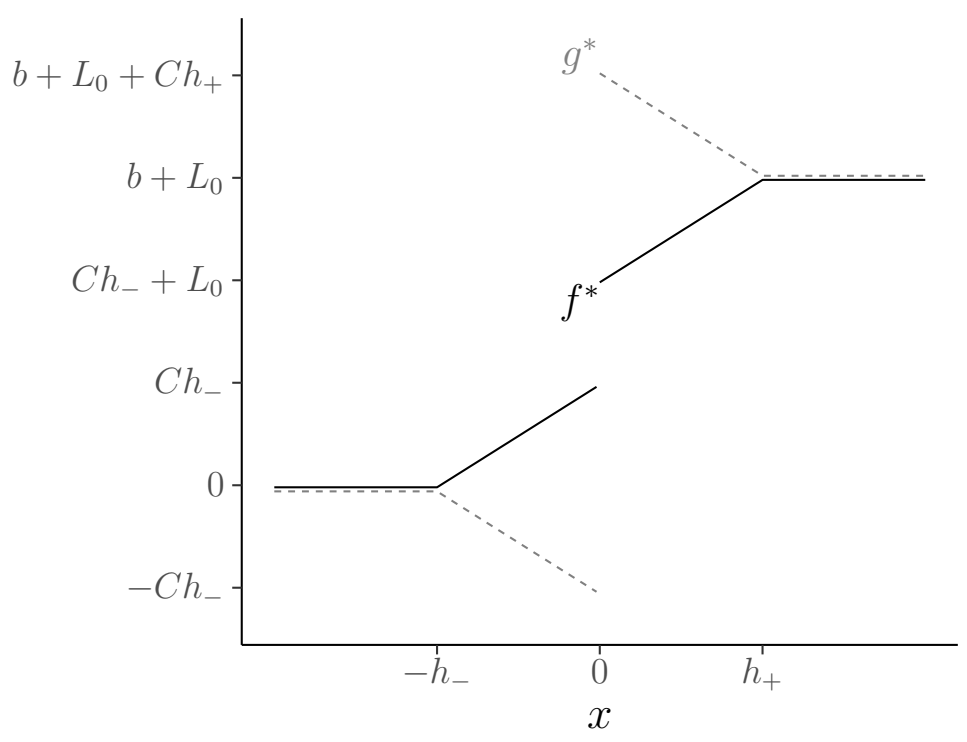

Figure 1: The least favorable null and alternative functions $f^{*}$ and $g^{*}$ from Equation (3) in Section 2.1.

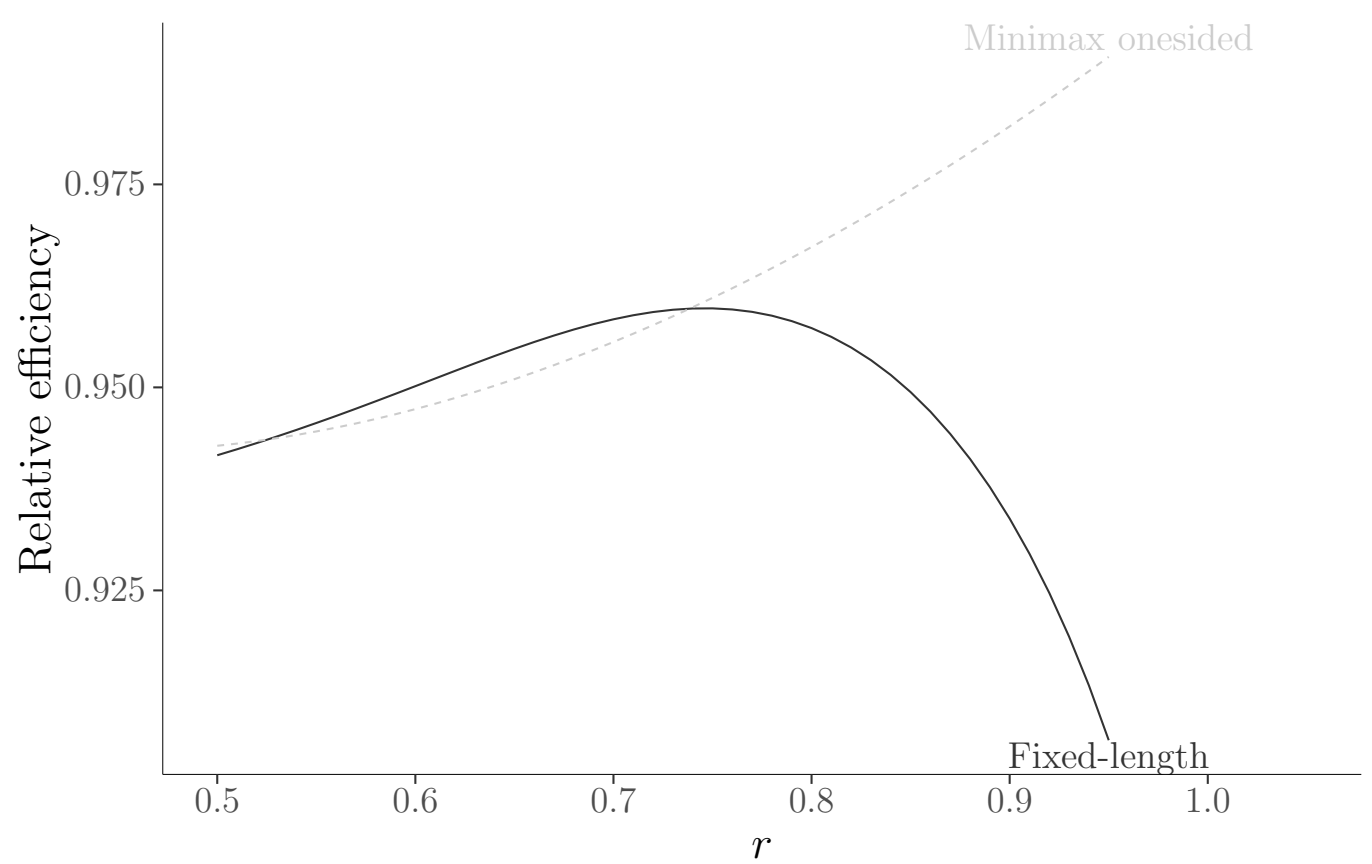

Figure 2: Asymptotic efficiency bounds for one-sided and fixed-length CIs as function of the optimal rate of convergence $r$ under centrosymmetry. Minimax one-sided refers to ratio of $\beta$-quantile of excess length of CIs that direct power at smooth functions relative to minimax one-sided CIs given in (28). Shortest fixed-length refers the ratio of expected length of CIs that direct power at a given smooth function relative to shortest fixed-length affine CIs given in Theorem E.1. 

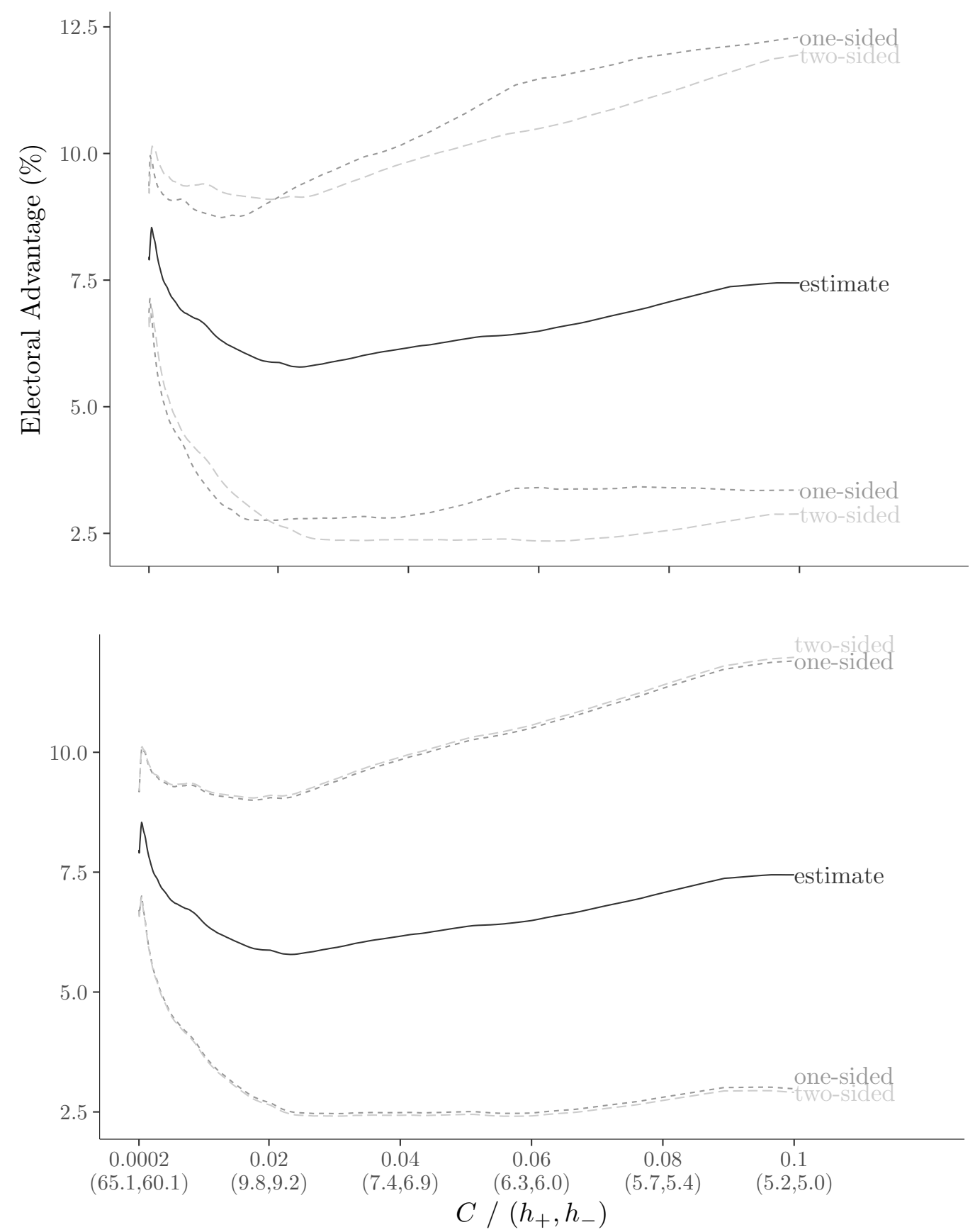

Figure 3: Lee (2008) RD example. Top panel displays minimax MSE estimator (estimator), and lower and upper limits of minimax one-sided confidence intervals for 0.8 quantile (onesided), and fixed-length CIs (two-sided) as function of smoothness $C$. Bottom panel displays one-and two-sided CIs around the minimax MSE estimator. $h_{+}, h_{-}$correspond to the optimal smoothness parameters for the minimax MSE estimator. 

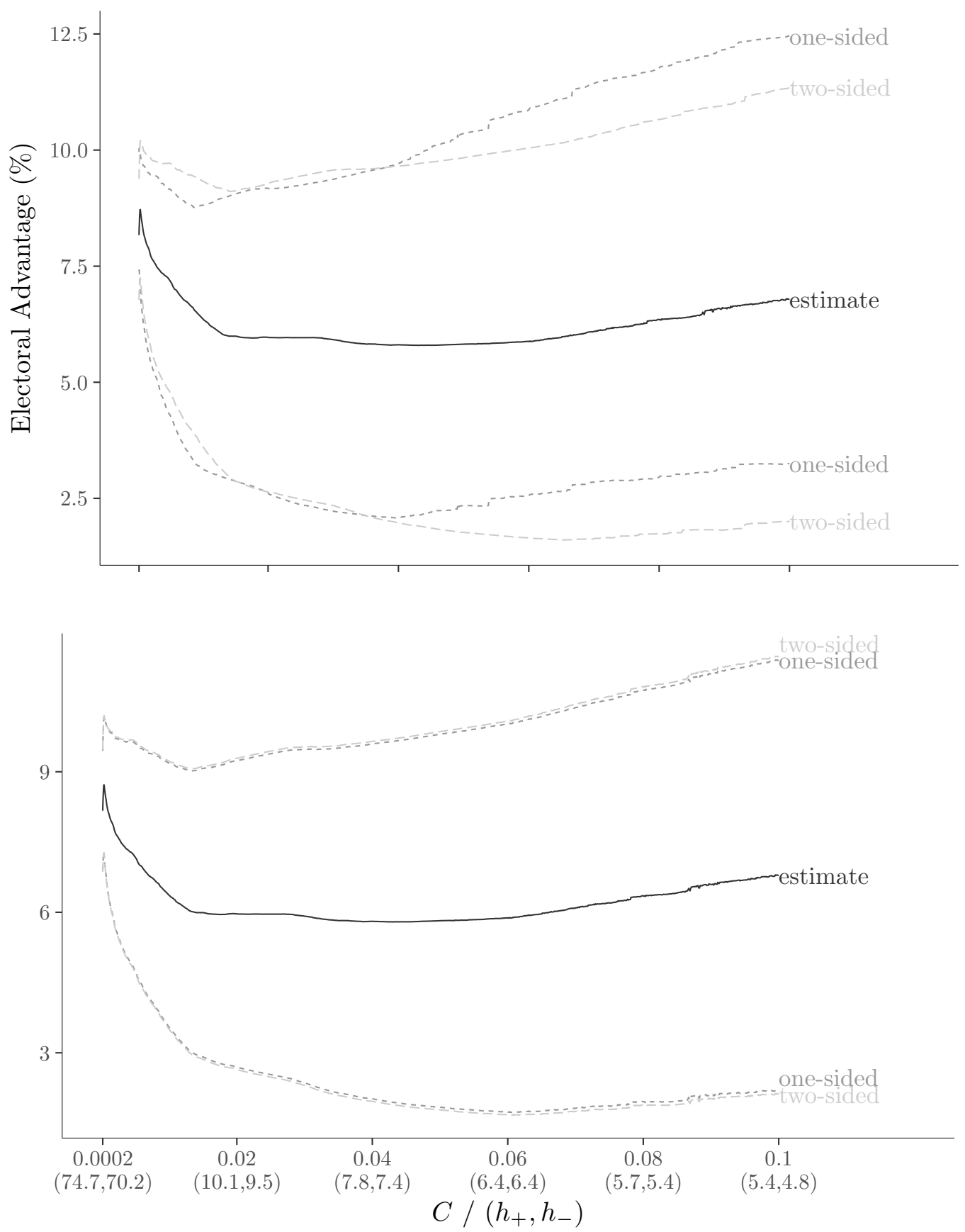

Figure 4: Lee (2008) RD example: local linear regression with triangular kernel. Top panel displays estimator based on minimax MSE bandwidths (estimator), lower and upper limits of one-sided CIs with bandwidths that are minimax for 0.8 quantile of excess length (onesided), and shortest fixed-length CIs (two-sided) as function of smoothness $C$. Bottom panel displays one-and two-sided CIs around and estimator based on minimax MSE bandwidths. $h_{+}, h_{-}$correspond to the minimax MSE bandwidths. 


\title{
Supplement to "Optimal inference in a class of regression models"
}

\author{
Timothy B. Armstrong* \\ Yale University
}

\author{
Michal Kolesár ${ }^{\dagger}$ \\ Princeton University
}

November 23, 2017

\begin{abstract}
This supplement provides appendices not included in the main text. Supplemental Appendix C compares our approach with other methods, and includes a Monte Carlo study. Supplemental Appendix D contains details for the results in Section 3 not included in the main text. Supplemental Appendix E contains details for the RD application. Supplemental Appendix F considers feasible versions of the procedures in Section 3 in the case with unknown error distribution and derives their asymptotic efficiency. Supplemental Appendix G gives some auxiliary results used for relative asymptotic efficiency comparisons. Supplemental Appendix H gives the proof of Theorem E.1.
\end{abstract}

\section{Appendix C Comparison with other methods}

This section compares the CIs developed in this paper to other approaches to inference in the RD application. We consider two popular approaches. The first approach is to form a nominal $100 \cdot(1-\alpha) \%$ CI by adding and subtracting the $1-\alpha / 2$ quantile of the $\mathcal{N}(0,1)$ distribution times the standard error, thereby ignoring any bias. We refer to these CIs as "conventional." The second approach is the robust bias correction (RBC) method studied by Calonico et al. (2014), which subtracts an estimate of the bias, and then takes into account the estimation error in this bias correction in forming the interval.

The coverage of these CIs will depend on the smoothness class $\mathcal{F}$ as well as the choice of bandwidth. Since CIs reported in applied work are typically based on local linear esti-

*email: timothy.armstrong@yale.edu

$\dagger^{\dagger}$ email: mkolesar@princeton.edu 
mators, with relative efficiency results for minimax MSE in the class $\mathcal{F}_{T, 2}\left(C, \mathbb{R}_{+}\right)$for estimation of $f(0)$ due to Cheng et al. (1997) often cited as justification, we focus on the class $\mathcal{F}_{R D T, 2}(C)$ when computing coverage (in Supplemental Appendix C.2, we consider classes that also impose bounds on smoothness away from the discontinuity point rather than just placing bounds on the error of the Taylor approximation around the discontinuity point). If the bandwidth choice is non-random, then finite sample coverage can be computed exactly when errors are normal with known variance. ${ }^{1}$ We take this approach in Supplemental Appendix C.1. If a data-driven bandwidth is used, computing finite sample coverage exactly becomes computationally prohibitive. We examine the coverage and relative efficiency of CIs with data driven bandwidths in a Monte Carlo study in Supplemental Appendix C.2.

\section{C.1 Exact coverage with nonrandom bandwidth}

For a given CI, we examine coverage in the classes $\mathcal{F}_{R D T, 2}(C)$ by asking "what is the largest value of $C$ for which this CI has good coverage?" Since the conventional CI ignores bias, there will always be some undercoverage, so we formalize this by finding the largest value of $C$ such that a nominal 95\% CI has true coverage 90\%. This calculation is easily done using the formulas in Section 3.2: the conventional approach uses the critical value $z_{0.975}=\mathrm{cv}_{0.05}(0)$ to construct a nominal $95 \% \mathrm{CI}$, while a valid $90 \%$ CI uses $\mathrm{cv}_{0.1}\left(\overline{\operatorname{bias}}_{\mathcal{F}_{R D T, 2}(C)}(\hat{L}) / \operatorname{se}(\hat{L})\right)$ (where $\hat{L}$ denotes the estimator and $\operatorname{se}(\hat{L})$ denotes its standard error), so we equate these two critical values and solve for $C$.

The resulting value of $C$ for which undercoverage is controlled will depend on the bandwidth. To provide a simple numerical comparison to commonly used procedures, we consider the (data-dependent) Imbens and Kalyanaraman $\left(2012\right.$, IK) bandwidth $\hat{h}_{I K}$ in the context of the Lee application considered in Section 4, but treat it as if it were fixed a priori. The IK bandwidth selector leads to $\hat{h}_{I K}=29.4$ for local linear regression with the triangular kernel. The conventional two-sided CI based on this bandwidth is given by $7.99 \pm 1.71$. Treating the bandwidth as nonrandom, it achieves coverage of at least $90 \%$ over $\mathcal{F}_{R D T, 2}(C)$ as long as $C \leq C_{\text {conv }}=0.0018$. This is a rather low value, lower than the lower bound estimate on $C$ from Supplemental Appendix E.3. It implies that even when $x=20 \%$, the prediction error based on a linear Taylor approximation to $f$ can be reduced by less than $1 \%$ by using the true conditional expectation.

\footnotetext{
${ }^{1}$ The resulting coverage calculations hold in an asymptotic sense with unknown error distribution in the same way that, for example, coverage calculations in Stock and Yogo (2005) are valid in an asymptotic sense in the instrumental variables setting.
} 
As an alternative to the conventional approach, one can use the robust-bias correction method studied in Calonico et al. (2014). Calonico et al. (2014) show that if the pilot bandwidth and the kernel used by the bias estimator equal those used by the local linear estimator of $L f$, this method is equivalent to running a quadratic instead of a linear local regression, and then using the usual CI. In the Lee application with IK bandwidth, this delivers the CI $6.68 \pm 2.52$, increasing the half-length substantially relative to the conventional CI. The maximum smoothness parameter under which these CIs have coverage at least $90 \%$ is given by $C_{R B C}=0.0023>C_{\text {conv }}$. By way of comparison, the optimal $95 \%$ fixed-length CIs at $C_{R B C}$ leads to a much narrower CI given by $7.70 \pm 2.11$.

While the CCT CI maintains good coverage for a larger smoothness constant than the conventional CI, both constants are rather small (equivalently, coverage is bad for moderate values of $C$ ). This is an artifact of the large realized value of $\hat{h}_{I K}$ : the CCT CI essentially "undersmooths" relative to a given bandwidth by making the bias-standard deviation ratio

smaller. Since $\hat{h}_{I K}$ is large to begin with, the amount of undersmoothing is not enough to make the procedure robust to moderate values of $C$. In fact, the IK bandwidth is generally quite sensitive to tuning parameter choices: we show in a Monte Carlo study in Supplemental Appendix C.2 that the CCT implementation of the IK bandwidth yields smaller bandwidths and achieves good coverage over a much larger set of functions, at the cost of larger length. In finite samples, the tuning parameters drive the maximum bias of the estimator, and hence its coverage properties, even though under standard pointwise asymptotics, the tuning parameters shouldn't affect coverage.

In contrast, if one performs the CCT procedure starting from a minimax MSE optimal bandwidth based on a known smoothness constant $C$, the asymptotic coverage will be quite good (above 94\%), although the CCT CI ends up being about 30\% longer than the optimal CI (see Armstrong and Kolesár, 2016b). Thus, while using a data driven bandwidth selector such as IK for inference can lead to severe undercoverage for smoothness classes used in RD (even if one undersmooths or bias-corrects as in CCT), procedures such as RBC can have good coverage if based on an appropriate bandwidth choice that is fixed ex ante.

\section{C.2 Monte Carlo evidence with random bandwidth}

Corollaries 3.2 and 3.3 imply that confidence intervals based on data-driven bandwidths must either undercover or else cannot be shorter than fixed-length CIs that assume worstcase smoothness. We now illustrate this implication with a Monte Carlo study.

We consider the RD setup from Section 2. To help separate the difficulty in constructing 
CIs for $L f$ due to unknown smoothness of $f$ from that due to irregular design points or heteroskedasticity, for all designs below, the distribution of $x_{i}$ is uniform on $[-1,1]$, and $u_{i}$ is independent of $x_{i}$, distributed $\mathcal{N}\left(0, \sigma^{2}\right)$. The sample size is $n=500$ in each case.

For $\sigma^{2}$, we consider two values, $\sigma^{2}=0.1295$, and $\sigma^{2}=4 \times 0.1295=0.518$. We consider conditional mean functions $f$ that lie in the smoothness class

$$
\mathcal{F}_{R D H, 2}(C)=\left\{f_{+}-f_{-}: f_{+} \in \mathcal{F}_{H, 2}\left(C ; \mathbb{R}_{+}\right), f_{-} \in \mathcal{F}_{H, 2}\left(C ; \mathbb{R}_{-}\right)\right\},
$$

where $\mathcal{F}_{H, p}(C ; \mathcal{X})$ is the second-order Hölder class, the closure of twice-differentiable functions with second derivative bounded by $2 C$, uniformly over $\mathcal{X}$ :

$$
\mathcal{F}_{H, p}(C ; \mathcal{X})=\left\{f:\left|f^{\prime}\left(x_{1}\right)-f^{\prime}\left(x_{2}\right)\right| \leq 2 C\left|x_{1}-x_{2}\right| \text { all } x_{1}, x_{2} \in \mathcal{X}\right\} .
$$

Unlike the class $\mathcal{F}_{R D T, 2}(C)$, the class $\mathcal{F}_{R D H, 2}(C)$ also imposes smoothness away from the cutoff, so that $\mathcal{F}_{R D H, 2}(C) \subseteq \mathcal{F}_{R D T, 2}(C)$. Imposing smoothness away from the cutoff is natural in many empirical applications. We consider $C=1$ and $C=3$, and for each $C$, we consider 4 different shapes for $f$. In each case, $f$ is odd, $f_{+}=-f_{-}$. In Designs 1 through 3 , $f_{+}$is given by a quadratic spline with two knots, at $b_{1}$ and $b_{2}$,

$$
f_{+}(x)=1(x \geq 0) \cdot C\left(x^{2}-2\left(x-b_{1}\right)_{+}^{2}+2\left(x-b_{2}\right)_{+}^{2}\right) .
$$

In Design 1 the knots are given by $\left(b_{1}, b_{2}\right)=(0.45,0.75)$, in Design 2 by $(0.25,0.65)$, and in Design 3 by $(0.4,0.9)$. The function $f_{+}(x)$ is plotted in Figure $\mathrm{S} 1$ for $C=1$. For $C=3$, the function $f$ is identical up to scale. It is clear from the figure that although locally to the cutoff, the functions are identical, they differ away from the cutoff (for $|x| \geq 0.25$ ), which, as we demonstrate below, affects the performance of data-driven methods. Finally, in Design 4, we consider $f(x)=0$ to allow us to compare the performance of CIs when $f$ is as smooth as possible.

We consider four methods for constructing CIs based on data-driven bandwidths, and two fixed-length CIs. All CIs are based on local polynomial regressions with a triangular kernel. The variance estimators used to construct the CIs are based on the nearest-neighbor method described in Remark 2.1. The results based on Eicker-Huber-White variance estimators are very similar and not reported here.

The first two methods correspond to conventional CIs based on local linear regression described in Supplemental Appendix C.1. The first CI uses Imbens and Kalyanaraman 
(2012, IK) bandwidth selector $\hat{h}_{I K}$, and the second CI uses a bandwidth selector proposed in Calonico et al. (2014, CCT), $\hat{h}_{C C T}$. The third CI uses the robust bias correction (RBC) studied in CCT, with both the pilot and the main bandwidth given by $\hat{h}_{I K}$ (the main estimate is based on local linear regression, and the bias correction is based on local quadratic regression), so that the bandwidth ratio is given by $\rho=1$. The fourth CI is also based on $\mathrm{RBC}$, but with the main and pilot bandwidth potentially different and given by the Calonico et al. (2014) bandwidth selectors. Finally, we consider two fixed-length CIs with uniform coverage under the class $\mathcal{F}_{R D H, 2}(C)$, with $C=1,3$, and bandwidth chosen to minimize their half-length. Their construction is similar to the CIs considered in Section 2.2, except they use the fact that under $\mathcal{F}_{R D H, 2}(C)$, the maximum bias for local linear estimators based on a fixed bandwidth is attained at $g^{*}(x)=C x^{2} 1(x \geq 0)-C x^{2} 1(x<0)$ (see Armstrong and Kolesár, 2016b, for derivation).

The results are reported in Table $\mathrm{S} 1$ for $C=1$ and $\mathrm{S} 2$ for $C=3$. One can see from the tables that CIs based on $\hat{h}_{I K}$ may undercover severely even at the higher level of smoothness, $C=1$. In particular, the coverage of conventional CIs based on $\hat{h}_{I K}$ is as low as $10.1 \%$ for $95 \%$ nominal CIs in Design 1, and the coverage of RBC CIs is as low as 64.4\%, again in Design 1. The undercoverage is even more severe when $C=3$.

In contrast, CIs based on the CCT bandwidth selector perform much better in terms of coverage under $C=1$, with coverage over $90 \%$ for all designs. These CIs only start undercovering once $C=3$, with $80.7 \%$ coverage in Design 3 for conventional CIs, and $86.2 \%$ coverage for RBC CIs. The cost for the good coverage properties, as can be seen from the tables, is that the CIs are longer, sometimes much longer than optimal fixed-length CIs.

As discussed in Supplemental Appendix C.1, the dramatically different coverage properties of the CIs based on the IK and CCT bandwidths illustrates the point that the coverage of CIs based on data-driven bandwidths is governed by the tuning parameters used in defining the bandwidth selector. These results can also be interpreted as showing the limits of procedures that try to "estimate $C$ " from the data. In particular, we show in Armstrong and Kolesár (2016b) that for inference at a point based on local linear regression under the second-order Hölder class, in large samples the MSE-optimal bandwidth (see Remark 2.2) differs from the usual (infeasible) bandwidth minimizing the large-sample MSE under pointwise asymptotics only in that it replaces $f^{\prime \prime}(0)$ with $C$. Thus, plug-in rules that estimate the infeasible pointwise bandwidth by plugging in an estimate of $f^{\prime \prime}(0)$ can be interpreted as data-driven bandwidths that try to estimate $C$ from the data. Since the IK and CCT bandwidths are plug-in rules, to the extent that one can interpret them as trying to "estimate $C$ " 
from the data, these simulation results also illustrate the point that attempts to estimate $C$ from the data cannot improve upon FLCIs (one can show that if these procedures were successful at estimating $C$, conventional CIs with $95 \%$ nominal level based on them should have coverage no less than $92.1 \%$ in large samples).

To assess sensitivity of these results to the normality and homoskedasticity of the errors, we also considered Designs 1-4 with heteroskedastic and log-normal errors. The results (not reported here) are similar in the sense that if a particular method achieved close to $95 \%$ coverage under normal homoskedastic errors, the coverage remained good under alternative error distributions. If a particular method undercovered in a given design, the amount of undercoverage could be more or less severe, depending on the form of heteroskedasticity. In particular, fixed-length CIs with $C=3$ achieve excellent coverage for all designs and all error distributions considered.

\section{Appendix D Additional details for Section 3}

This section contains details for the results in Section 3 not included in the main text.

\section{D.1 Special cases}

In addition to regression discontinuity, the regression model (1) covers several other important models, including inference at a point $\left(L f=f\left(x_{0}\right)\right.$ with $x_{0}$ given $)$ and average treatment effects under unconfoundedness (with $L f=\frac{1}{n} \sum_{i=1}^{n}\left(f\left(w_{i}, 1\right)-f\left(w_{i}, 0\right)\right)$ where $x_{i}=\left(w_{i}^{\prime}, d_{i}\right)^{\prime}$, $d_{i}$ is a treatment indicator and $w_{i}$ are controls).

The setup (18) can also be used to study the linear regression model with restricted parameter space. For simplicity, consider the case with homoskedastic errors,

$$
Y=X \theta+\sigma \varepsilon, \quad \varepsilon \sim \mathcal{N}\left(0, I_{n}\right)
$$

where $X$ is a fixed $n \times k$ design matrix and $\sigma$ is known. This fits into our framework with $f=\theta, X$ playing the role of $K$, taking $\theta \in \mathbb{R}^{k}$ to $X \theta \in \mathbb{R}^{n}$, and $\mathcal{Y}=\mathbb{R}^{n}$ with the Euclidean inner product $\langle x, y\rangle=x^{\prime} y$. We are interested in a linear functional $L \theta=\ell^{\prime} \theta$ where $\ell \in \mathbb{R}^{k}$. We consider this model in previous version of this paper (Armstrong and Kolesár, 2016a). Furthermore, (18) covers the multivariate normal location model $\hat{\theta} \sim \mathcal{N}(\theta, \Sigma)$, which obtains as a limiting experiment of regular parametric models. Our finite-sample results could thus be extended to local asymptotic results in regular parametric models with restricted parameter 
spaces.

In addition to the regression models (1) and (S1), the setup (18) includes other nonparametric and semiparametric regression models such as the partly linear model (where $f$ takes the form $g\left(w_{1}\right)+\gamma^{\prime} w_{2}$, and we are interested in a linear functional of $g$ or $\gamma$ ). It also includes the Gaussian white noise model, which can be obtained as a limiting model for nonparametric density estimation (see Nussbaum, 1996) as well as nonparametric regression with fixed or random regressors (see Brown and Low, 1996; Reiß, 2008). These white noise equivalence results imply that our finite-sample results translate to asymptotic results in problems such as inference at a point in density estimation or regression with random regressors. We refer the reader to Donoho (1994, Section 9) for details of these and other models that fit into the general setup (18).

\section{D.2 Derivative of the modulus}

The class of optimal estimators $\hat{L}_{\delta, \mathcal{F}, \mathcal{G}}$ involves the superdifferential of the modulus. In the case where the modulus is differentiable, the superdifferential is a singleton, so that $\hat{L}_{\delta, \mathcal{F}, \mathcal{G}}$ is defined uniquely. In this section, we introduce a condition that guarantees differentiability and leads to a formula for the derivative. We also briefly discuss the case where the modulus is not differentiable.

Definition 1 (Translation Invariance). The function class $\mathcal{F}$ is translation invariant if there exists a function $\iota \in \mathcal{F}$ such that $L \iota=1$ and $f+c \iota \in \mathcal{F}$ for all $c \in \mathbb{R}$ and $f \in \mathcal{F}$.

Translation invariance will hold in most cases where the parameter of interest $L f$ is unrestricted. For example, if $L f=f(0)$, it will hold with $\iota(x)=1$ if $\mathcal{F}$ places monotonicity restrictions and/or restrictions on the derivatives of $f$. Under translation invariance, the modulus is differentiable, and we obtain an explicit expression for its derivative:

Lemma D.1. Let $f^{*}$ and $g^{*}$ solve the modulus problem with $\delta_{0}=\left\|K\left(g^{*}-f^{*}\right)\right\|>0$, and suppose that $f^{*}+c \iota \in \mathcal{F}$ for all $c$ in a neighborhood of zero, where $L \iota=1$. Then the modulus is differentiable at $\delta_{0}$ with $\omega^{\prime}\left(\delta_{0} ; \mathcal{F}, \mathcal{G}\right)=\delta_{0} /\left\langle K \iota, K\left(g_{\delta_{0}}^{*}-f_{\delta_{0}}^{*}\right)\right\rangle$.

Proof. Let $d \in \partial \omega\left(\delta_{0} ; \mathcal{F}, \mathcal{G}\right)$ and let $f_{c}=f^{*}-c \iota$. Let $\eta$ be small enough so that $f_{c} \in \mathcal{F}$ for $|c| \leq \eta$. Then, for $|c| \leq \eta$,

$L\left(g^{*}-f^{*}\right)+d\left[\left\|K\left(g^{*}-f_{c}\right)\right\|-\delta_{0}\right] \geq \omega\left(\left\|K\left(g^{*}-f_{c}\right)\right\| ; \mathcal{F}, \mathcal{G}\right) \geq L\left(g^{*}-f_{c}\right)=L\left(g^{*}-f^{*}\right)+c$ 
where the first inequality follows from the definition of the superdifferential and the second inequality follows from the definition of the modulus. Since the left-hand side of the above display is greater than or equal to the right-hand side for $|c| \leq \eta$, and the two sides are equal at $c=0$, the derivatives of both sides with respect to $c$ must be equal. Since

$$
\left.\frac{d\left\|K\left(g^{*}-f_{c}\right)\right\|}{d c}\right|_{c=0}=\frac{\left.\frac{d}{d c}\left\|K\left(g^{*}-f_{c}\right)\right\|^{2}\right|_{c=0}}{2 \delta_{0}}=\frac{\left\langle K\left(g^{*}-f^{*}\right), K \iota\right\rangle}{\delta_{0}}
$$

result follows.

The explicit expression for $\omega^{\prime}(\delta ; \mathcal{F}, \mathcal{G})$ is useful in simplifying the expressions $(23)$ and $(25)$ for the optimal estimators.

Translation invariance leads to a direct relation between optimal CIs and tests. In general, it can be seen from Lemma A.2 that the test that rejects $L_{0}$ when $L_{0} \notin\left[\hat{c}_{\alpha, \delta, \mathcal{F}, \mathcal{G}}, \infty\right)$ is minimax for $H_{0}: L f \leq L_{0}$ and $f \in \mathcal{F}$ against $H_{1}: L f \geq L_{0}+\omega(\delta ; \mathcal{F}, \mathcal{G})$ and $f \in \mathcal{G}$, where $L_{0}=L f_{\delta}^{*}$. If both $\mathcal{F}$ and $\mathcal{G}$ are translation invariant, $f_{\delta}^{*}+c \iota$ and $g_{\delta}^{*}+c \iota$ achieve the ordered modulus for any $c \in \mathbb{R}$, so that, varying $c$, this test can be seen to be minimax for any $L_{0}$. Thus, under translation invariance, the CI in Theorem 3.1 inverts minimax one sided tests with distance to the null given by $\omega(\delta)$ (in general, the test based on the CI in Theorem 3.1 is minimax only when $L_{0}=L f_{\delta}^{*}$ ).

If the modulus is not differentiable at some $\delta$, the CIs defined in Sections 3.3 and 3.4 are valid with $\omega^{\prime}(\delta, \mathcal{F}, \mathcal{G})$ given by any element of the superdifferential, so long as the same element of the superdifferential is used throughout the formula (in particular, the same element used in the estimator (23) must be used in the worst-case bias formula (24)). For the one-sided CI, Theorem 3.1 applies regardless of which element of the superdifferential is used. In the two-sided case, when computing the optimal fixed-length affine CI described in Section 3.4, the only additional detail in the case where the modulus is not everywhere differentiable is that one optimizes the half-length over both $\delta$ and over elements in the superdifferential.

\section{Appendix E Additional details for RD}

This section gives additional details for the RD application. Supplemental Appendix E.1 derives the worst-case bias formula given in (11). Supplemental Appendix E.2 derives the optimal estimator and the solution to the modulus problem. Supplemental Appendix E.3 discusses lower bounds for the smoothness constant $C$. Supplemental Appendix E.4 shows 
the asymptotic validity of the feasible version of the estimator in which the variance is estimated.

\section{E.1 Worst-case bias for linear estimators}

This section derives the worst-case bias formula (11) for linear estimators $\hat{L}_{h_{+}, h_{-}}$defined in $(10)$ in Section 2.2. We require the weights to satisfy $w_{+}\left(-x, h_{+}\right)=w_{-}\left(x, h_{-}\right)=0$ for $x \geq 0$ and

$$
\begin{aligned}
\sum_{i=1}^{n} w_{+}\left(x_{i}, h_{+}\right) & =\sum_{i=1}^{n} w_{-}\left(x_{i}, h_{-}\right)=1, \\
\sum_{i=1}^{n} x_{i}^{j} w_{-}\left(x_{i}, h_{-}\right) & =\sum_{i=1}^{n} x_{i}^{j} w_{+}\left(x_{i}, h_{+}\right)=0 \text { for } j=1, \ldots, p-1 .
\end{aligned}
$$

Note that (S2) holds iff. $\hat{L}_{h_{+}, h_{-}}$is unbiased at all $f=f_{+}+f_{-}$where $f_{+}$and $f_{-}$are both polynomials of order $p-1$ or less, which is necessary to ensure that the worst-case bias is finite. This condition holds if $\hat{L}_{h_{+}, h_{-}}$is based on a local polynomial estimator of order at least $p-1$.

We can write any function $f \in \mathcal{F}_{R D T, p}(C)$ as $f=f_{+}+f_{-}$, where

$$
f_{+}(x)=\left[\sum_{j=0}^{p-1} f_{+}^{(j)}(0) x^{j} / j !+r_{+}(x)\right] 1(x \geq 0), \quad f_{-}(x)=\left[\sum_{j=0}^{p-1} f_{-}^{(j)}(0) x^{j} / j !+r_{-}(x)\right] 1(x<0),
$$

and the remainder terms $r_{+}$and $r_{-}$satisfy $\left|r_{+}(x)\right| \leq C|x|^{p}$ and $\left|r_{-}(x)\right| \leq C|x|^{p}$. Under (S2), we can therefore write

$$
\operatorname{bias}_{f}\left(\hat{L}_{h_{+}, h_{-}}\right)=\sum_{i=1}^{n} w_{+}\left(x_{i}, h_{+}\right) r_{+}(x)-\sum_{i=1}^{n} w_{-}\left(x_{i}, h_{+}\right) r_{-}(x),
$$

which is maximized subject to the conditions $\left|r_{+}(x)\right| \leq C|x|^{p}$ and $\left|r_{-}(x)\right| \leq C|x|^{p}$ by taking $r_{+}\left(x_{i}\right)=C\left|x_{i}\right|^{p} \cdot \operatorname{sign}\left(w_{+}\left(x_{i}, h_{+}\right)\right)$and $r_{-}\left(x_{i}\right)=-C\left|x_{i}\right|^{p} \cdot \operatorname{sign}\left(w_{-}\left(x_{i}, h_{-}\right)\right)$. This yields the worst-case bias formula Equation (11).

\section{E.2 Solution to the modulus problem and optimal estimators}

This section derives the form of the optimal estimators and CIs. To that end, we first need to find functions $g_{\delta}^{*}$ and $f_{\delta}^{*}$ that solve the modulus problem. Since the class $\mathcal{F}_{R D T, p}(C)$ is centrosymmetric, $f_{\delta}^{*}=-g_{\delta}^{*}$, and the (single-class) modulus of continuity $\omega\left(\delta ; \mathcal{F}_{R D T, p}(C)\right)$ is 
given by the value of the problem

$$
\sup _{f_{+}+f_{-} \in \mathcal{F}_{R D T, p}(C)} 2\left(f_{+}(0)-f_{-}(0)\right) \quad \text { st } \quad \sum_{i=1}^{n} \frac{f_{-}\left(x_{i}\right)^{2}}{\sigma^{2}\left(x_{i}\right)}+\sum_{i=1}^{n} \frac{f_{+}\left(x_{i}\right)^{2}}{\sigma^{2}\left(x_{i}\right)} \leq \delta^{2} / 4
$$

Let $g_{\delta, C}^{*}$ denote the (unique up to the values at the $x_{i}$ s) solution to this problem. This solution can be obtained using a simple generalization of Theorem 1 of Sacks and Ylvisaker (1978). To describe it, define $g_{b, C}(x)=g_{+, b, C}(x)+g_{-, b, C}(x)$ by

$$
\begin{aligned}
& g_{+, b, C}(x)=\left(\left(b-b_{-}+\sum_{j=1}^{p-1} d_{+, j} x^{j}-C|x|^{p}\right)_{+}-\left(b-b_{-}+\sum_{j=1}^{p-1} d_{+, j} x^{j}+C|x|^{p}\right)_{-}\right) 1(x \geq 0), \\
& g_{-, b, C}(x)=-\left(\left(b_{-}+\sum_{j=1}^{p-1} d_{-} x^{j}-C|x|^{p}\right)_{+}-\left(b_{-}+\sum_{j=1}^{p-1} d_{-, j} x^{j}+C|x|^{p}\right)_{-}\right) 1(x<0),
\end{aligned}
$$

where we use the notation $(t)_{+}=\max \{t, 0\}$ and $(t)_{-}=-\min \{t, 0\}$. The solution is given by $g_{\delta, C}^{*}=g_{b(\delta), C}$ where the coefficients $d_{+}=\left(d_{+, 1}, \ldots, d_{-, p-1}\right), d_{-}=\left(d_{-, 1}, \ldots, d_{-, p-1}\right)$, and $b(\delta)$ and $b_{-}$solve a system of equations given below. To see that the solution must take the form $g_{b, C}(x)$ for some $b, b_{-}, d_{+}, d_{-}$, note that any function $f_{+} \in \mathcal{F}_{T, p}(C)$ can be written as

$$
f_{+}(x)=b_{+}+\sum_{j=1}^{p-1} d_{+, j} x^{j}+r_{+}(x), \quad\left|r_{+}(x)\right| \leq C|x|^{p}
$$

Given $b_{+}, d_{+}$, in order to minimize $\left|f_{+}\left(x_{i}\right)\right|$ simultaneously for all $i$, it must be that

$$
r_{+}(x)= \begin{cases}-C|x|^{p} & \text { if } b_{+}+\sum_{j=1}^{p=1} d_{+, j} x^{j} \geq C|x|^{p}, \\ -b_{+}-\sum_{j=1}^{p=1} d_{+, j} x^{j} & \text { if }\left|b_{+}+\sum_{j=1}^{p=1} d_{+, j} x^{j}\right|<C|x|^{p}, \\ C|x|^{p} & \text { if } b_{+}+\sum_{j=1}^{p=1} d_{+, j} x^{j} \leq-C|x|^{p} .\end{cases}
$$

This form of $r(x)$ is necessary for $f_{+}$to solve (S3): otherwise, one could strictly decrease $\sum_{i=1}^{n}\left[f_{-}\left(x_{i}\right)^{2} / \sigma^{2}\left(x_{i}\right)+f_{+}\left(x_{i}\right)^{2} / \sigma^{2}\left(x_{i}\right)\right]$, thereby making this quantity strictly less than $\delta^{2} / 4$. But this would allow for a strictly larger value of $2\left(f_{+}(0)+f_{-}(0)\right)$ by increasing $b_{+}$and leaving $d_{+}$and $r_{+}$the same. Plugging $r_{+}(x)$ from the above display into (S4) shows that $f_{+}(x)=g_{+, b, C}(x)$ for some $b_{+}, d_{+}$. Similar arguments apply for $f_{-}$.

Setting up the Lagrangian for the problem with $f$ constrained to the class of functions that take the form $g_{b, C}$ for some $b, b_{-}, d_{+}, d_{-}$, and taking first order conditions with respect 
to $b_{-}, d_{+}$and $d_{-}$gives

$$
\begin{aligned}
& 0=\sum_{i=1}^{n} \frac{g_{-, b, C}\left(x_{i}\right)}{\sigma^{2}\left(x_{i}\right)}\left(x_{i}, \ldots, x_{i}^{p-1}\right)^{\prime}, \\
& 0=\sum_{i=1}^{n} \frac{g_{+, b, C}\left(x_{i}\right)}{\sigma^{2}\left(x_{i}\right)}\left(x_{i}, \ldots, x_{i}^{p-1}\right)^{\prime}, \\
& 0=\sum_{i=1}^{n} \frac{g_{+, b, C}\left(x_{i}\right)}{\sigma^{2}\left(x_{i}\right)}+\sum_{i=1}^{n} \frac{g_{-, b, C}\left(x_{i}\right)}{\sigma^{2}\left(x_{i}\right)} .
\end{aligned}
$$

The constraint in (S3) must be binding at the optimum, which gives the additional equation

$$
\delta^{2} / 4=\sum_{i=1}^{n} \frac{g_{b, C}\left(x_{i}\right)^{2}}{\sigma^{2}\left(x_{i}\right)}=b \sum_{i=1}^{n} \frac{g_{+, b, C}\left(x_{i}\right)}{\sigma^{2}\left(x_{i}\right)}-C \sum_{i=1}^{n} \frac{\left|g_{b, C}\left(x_{i}\right)\right|\left|x_{i}\right|^{p}}{\sigma^{2}\left(x_{i}\right)}
$$

where the second equality follows from (S5)-(S6). Note also that, since $g_{\delta, C}^{*}=g_{b(\delta), C}$ solves the modulus problem and gives the modulus as $2 b(\delta)$, it also gives the solution to the inverse modulus problem

$$
\frac{\omega^{-1}\left(2 b ; \mathcal{F}_{R D T, p}(C)\right)^{2}}{4}=\inf _{f_{+}-f_{-} \in \mathcal{F}_{R D T, p}(C)} \sum_{i=1}^{n}\left(\frac{f_{+}^{2}\left(x_{i}\right)}{\sigma^{2}\left(x_{i}\right)}+\frac{f_{-}^{2}\left(x_{i}\right)}{\sigma^{2}\left(x_{i}\right)}\right) \text { s.t. } 2\left(f_{+}(0)-f_{-}(0)\right) \geq 2 b
$$

for $b=b(\delta)$. Since the objective for the inverse modulus is strictly convex, this shows that the solution is unique up to the values at the $x_{i}$ s.

Using the fact that the class $\mathcal{F}_{R D T, p}(C)$ is translation invariant as defined in Supplemental Appendix D.2 (we can take $\iota(x)=c_{0}+1\left(x \geq 0\right.$ ) for any $\left.c_{0}\right)$, so that the derivative of the modulus is given by Lemma D.1, along with (S7) implies that the class of estimators $\hat{L}_{\delta}$ can be written as

$$
\hat{L}_{\delta}=\hat{L}_{\delta, \mathcal{F}_{R D T, p}(C)}=\frac{\sum_{i=1}^{n} g_{+, \delta, C}^{*}\left(x_{i}\right) y_{i} / \sigma^{2}\left(x_{i}\right)}{\sum_{i=1}^{n} g_{+, \delta, C}^{*}\left(x_{i}\right) / \sigma^{2}\left(x_{i}\right)}-\frac{\sum_{i=1}^{n} g_{-, \delta, C}^{*}\left(x_{i}\right) y_{i} / \sigma^{2}\left(x_{i}\right)}{\sum_{i=1}^{n} g_{-, \delta, C}^{*}\left(x_{i}\right) / \sigma^{2}\left(x_{i}\right)} .
$$

Note that Conditions (S5), (S6), and (S7) are simply the conditions (S2) applied to this class of estimators.

To write the estimator $\hat{L}_{\delta}$ in the form (10), let $w_{-}\left(x_{i}, h_{-}\right)=g_{-, b, C}\left(x_{i}\right) / \sum_{i=1}^{n} g_{-, b, C}\left(x_{i}\right)$ and $w_{+}\left(x_{i}, h_{+}\right)=g_{+, b, C}\left(x_{i}\right) / \sum_{i=1}^{n} g_{+, b, C}\left(x_{i}\right)$, where $d_{+}$and $d_{-}$solve (S5) and (S6) with $b-b_{-}=C h_{+}^{p}$ and $b_{-}=C h_{-}^{p}$. Then $\hat{L}_{\delta}=\hat{L}_{h_{+}(\delta), h_{-}(\delta)}$ where $h_{+}(\delta)$ and $h_{-}(\delta)$ are determined by the additional conditions $(\mathrm{S} 7)$ and $(\mathrm{S} 8)$. 
To find the optimal estimators as described in Section 2.2, one can use the estimator $\hat{L}_{h_{+}, h_{-}}$and optimize $h_{+}$and $h_{-}$for the given performance criterion, using the variance and worst-case bias formulas given in that section. Since the optimal estimator $\hat{L}_{\delta}$ (with $\delta$ determined by the performance criterion) takes this form for some $h_{+}$and $h_{-}$, the resulting estimator and CI will be the same as the one obtained by computing $\hat{L}_{\delta}$ with $\delta$ determined by solving the additional equation that corresponds to the performance criterion of interest.

\section{E.3 Lower bound on $C$}

While it is not possible to consistently estimate the smoothness constant $C$ from the data, it is possible to lower bound its value. Here we develop a simple estimator and lower CI for this bound, focusing on the case $f \in \mathcal{F}_{R D T, 2}(C)$.

As noted in Supplemental Appendix E.2, we can write $f_{+}(x)=f_{+}(0)+f_{+}^{\prime}(0) x+r_{+}(x)$, where $\left|r_{+}(x)\right| \leq C x^{2}$. It therefore follows that for any three points $0 \leq x_{1} \leq x_{2} \leq x_{3}$,

$$
\lambda f_{+}\left(x_{1}\right)+(1-\lambda) f_{+}\left(x_{3}\right)-f_{+}\left(x_{2}\right)=\lambda r_{+}\left(x_{1}\right)+(1-\lambda) r_{+}\left(x_{3}\right)-r_{+}\left(x_{2}\right),
$$

where $\lambda=\left(x_{3}-x_{2}\right) /\left(x_{3}-x_{1}\right)$. The left-hand side measures the curvature of $f$ by comparing $f\left(x_{2}\right)$ to an approximation based on linearly interpolating between $f\left(x_{1}\right)$ and $f\left(x_{3}\right)$. Since $\left|r_{+}(x)\right| \leq C x^{2}$, the right-hand side is bounded by $C\left(\lambda x_{1}^{2}+(1-\lambda) x_{3}^{3}+x_{2}^{2}\right)$. Taking averages of the preceding display over intervals $I_{k}=\left[a_{k-1}, a_{k}\right)$ where $a_{0} \leq a_{1} \leq a_{2} \leq a_{3}$ and applying this bound yields the lower bound

$$
C \geq\left|\mu_{+}\right|, \quad \mu_{+}=\frac{\lambda E_{n, 1}\left(f_{+}(x)\right)+(1-\lambda) E_{n, 3}\left(f_{+}(x)\right)-E_{n, 2}\left(f_{+}(x)\right)}{\lambda E_{n, 1}\left(x^{2}\right)+(1-\lambda) E_{n, 3}\left(x^{2}\right)+E_{n, 2}\left(x^{2}\right)},
$$

where we use the notation $E_{n, k}(g(x))=\sum_{i} 1\left(x_{i} \in I_{k}\right) g\left(x_{i}\right) / n_{k}, n_{k}=\sum_{i} 1\left(x_{i} \in I_{k}\right) g\left(x_{i}\right)$ to denote sample average over $I_{k}$. Replacing $E_{n, k}\left(f_{+}(x)\right)$ with $E_{n, k}(y)$ yields the estimator of $\mu_{+}$

$$
Z=\frac{\lambda E_{n, 1}(y)+(1-\lambda) E_{n, 3}(y)-E_{n, 2}(y)}{\lambda E_{n, 1}\left(x^{2}\right)+(1-\lambda) E_{n, 3}\left(x^{2}\right)+E_{n, 2}\left(x^{2}\right)} \sim \mathcal{N}\left(\mu_{+}, \tau^{2}\right),
$$

where $\tau^{2}=\frac{\lambda^{2} E_{n, 1}\left(\sigma^{2}(x)\right) / n_{1}+(1-\lambda)^{2} E_{n, 3}\left(\sigma^{2}(x)\right) / n_{3}-E_{n, 2}\left(\sigma^{2}(x)\right) / n_{2}}{\left(\lambda E_{n, 1}\left(x^{2}\right)+(1-\lambda) E_{n, 3}\left(x^{2}\right)+E_{n, 2}\left(x^{2}\right)\right)^{2}}$. Inverting tests of the hypotheses $H_{0}:\left|\mu_{+}\right| \leq \mu_{0}$ against $H_{1}:\left|\mu_{+}\right|>\mu_{0}$ then yields a one-sided CI for $\left|\mu_{+}\right|$of the form $\left[\hat{\mu}_{+, \alpha}, \infty\right)$, where $\hat{\mu}_{+, \alpha}$ solves $|Z / \tau|=\mathrm{cv}_{\alpha}(\mu / \tau)$, with the convention that $\hat{\mu}_{+, \alpha}=0$ if $|Z / \tau| \leq \mathrm{cv}_{\alpha}(0)$. This CI can be used as a lower CI for $C$ in model specification checks.

Since unbiased estimates of the lower bound $\left|\mu_{+}\right|$do not exist, following Chernozhukov 
et al. (2013), we take $\hat{\mu}_{+, 0.5}$ as an estimator of the lower bound, which has the property that it's half-median unbiased in the sense that $P\left(\left|\mu_{+}\right| \leq \hat{\mu}_{+, 0.5}\right) \leq 0.5$. An analogous bound obtains by considering intervals below the cutoff. We leave the question of optimal choice of the intervals $I_{k}$ to future research. In the Lee (2008) application, we set $a_{0}=0$, and set the remaining interval endpoints $a_{k}$ such that each interval $I_{k}$ contains 200 observations. This yields estimates $\hat{\mu}_{+, 0.5}=0.008$ and $\hat{\mu}_{-, 0.5}=0.017$.

\section{E.4 Asymptotic validity}

We now give a theorem showing asymptotic validity of CIs from Section 2.2 under an unknown error distribution. We consider uniform validity over regression functions in $\mathcal{F}$ and error distributions in a sequence $\mathcal{Q}_{n}$, and we index probability statements with $f \in \mathcal{F}$ and $Q \in \mathcal{Q}_{n}$. We make the following assumptions on the $x_{i}$ s and the class of error distributions $\mathcal{Q}_{n}$.

Assumption E.1. For some $p_{X,+}(0)>0$ and $p_{X,-}(0)>0$, the sequence $\left\{x_{i}\right\}_{i=1}^{n}$ satisfies $\frac{1}{n h_{n}} \sum_{i=1}^{n} m\left(x_{i} / h_{n}\right) 1\left(x_{i} \geq 0\right) \rightarrow p_{X,+}(0) \int_{0}^{\infty} m(u) d u$ and $\frac{1}{n h_{n}} \sum_{i=1}^{n} m\left(x_{i} / h_{n}\right) 1\left(x_{i}<0\right) \rightarrow$

$p_{X,-}(0) \int_{-\infty}^{0} m(u) d u$ for any bounded function $m$ with bounded support and any $h_{n}$ with $0<\liminf _{n} h_{n} n^{1 /(2 p+1)} \leq \limsup _{n} h_{n} n^{1 /(2 p+1)}<\infty$.

Assumption E.2. For some $\sigma(x)$ with $\lim _{x \downarrow 0} \sigma(x)=\sigma_{+}(0)>0$ and $\lim _{x \uparrow 0} \sigma(x)=\sigma_{-}(0)>$ 0

(i) the $u_{i} s$ are independent under any $Q \in \mathcal{Q}_{n}$ with $E_{Q} u_{i}=0, \operatorname{var}_{Q}\left(u_{i}\right)=\sigma^{2}\left(x_{i}\right)$

(ii) for some $\eta>0, E_{Q}\left|u_{i}\right|^{2+\eta}$ is bounded uniformly over $n$ and $Q \in \mathcal{Q}_{n}$.

While the variance function $\sigma^{2}(x)$ is unknown, the definition of $\mathcal{Q}_{n}$ is such that the variance function is the same for all $Q \in \mathcal{Q}_{n}$. This is done for simplicity. One could consider uniformity over classes $\mathcal{Q}_{n}$ that place only smoothness conditions on $\sigma^{2}(x)$ at the cost of introducing additional notation and making the optimality statements more cumbersome.

The estimators and CIs that we consider in the sequel are based on an estimate $\hat{\sigma}(x)$ of the conditional variance in Step 1 of the procedure in Section 2.2. We make the following assumption on this estimate.

Assumption E.3. The estimate $\hat{\sigma}(x)$ is given by $\hat{\sigma}(x)=\hat{\sigma}_{+}(0) 1(x \geq 0)+\hat{\sigma}_{-}(0) 1(x<0)$ where $\hat{\sigma}_{+}(0)$ and $\hat{\sigma}_{-}(0)$ are consistent for $\sigma_{+}(0)$ and $\sigma_{-}(0)$ uniformly over $f \in \mathcal{F}$ and $Q \in \mathcal{Q}_{n}$. 
For asymptotic coverage, we consider uniformity over both $\mathcal{F}$ and $\mathcal{Q}_{n}$. Thus, a confidence set $\mathcal{C}$ is said to have asymptotic coverage at least $1-\alpha$ if

$$
\liminf _{n \rightarrow \infty} \inf _{f \in \mathcal{F}, Q \in \mathcal{Q}_{n}} P_{f, Q}(L f \in \mathcal{C}) \geq 1-\alpha
$$

Theorem E.1. Under Assumptions E.1, E.2 and E.3, CIs given in Section 2.2 based on $\hat{L}_{\delta}$ have asymptotic coverage at least $1-\alpha$. CIs based on local polynomial estimators have asymptotic coverage at least $1-\alpha$ so long as the kernel is bounded and uniformly continuous with bounded support and the bandwidths $h_{+}$and $h_{-}$satisfy $h_{+} n^{1 /(2 p+1)} \rightarrow h_{+, \infty}$ and $h_{-} n^{1 /(2 p+1)} \rightarrow h_{-, \infty}$ for some $h_{+, \infty}>0$ and $h_{-, \infty}>0$.

Let $\hat{\chi}$ denote the half-length of the optimal fixed-length CI based on $\hat{\sigma}(x)$. For $\chi_{\infty}$ given in Supplemental Appendix $H$, the scaled half-length $n^{p /(2 p+1)} \hat{\chi}$ converges in probability to $\chi_{\infty}$ uniformly over $\mathcal{F}$ and $\mathcal{Q}_{n}$. If, in addition, each $\mathcal{Q}_{n}$ contains a distribution where the $u_{i} s$ are normal, then for any sequence of confidence sets $\mathcal{C}$ with asymptotic coverage at least $1-\alpha$, we have the following bound on the asymptotic efficiency improvement at any $f \in \mathcal{F}_{R D T, p}(0)$

$$
\liminf _{n \rightarrow \infty} \sup _{Q \in \mathcal{Q}_{n}} \frac{n^{p /(2 p+1)} E_{f, Q} \lambda(\mathcal{C})}{2 \chi_{\infty}} \geq \frac{(1-\alpha) 2^{r} E\left[\left(z_{1-\alpha}-Z\right)^{r} \mid Z \leq z_{1-\alpha}\right]}{2 r \inf _{\delta>0} \operatorname{cv}_{\alpha}((\delta / 2)(1 / r-1)) \delta^{r-1}}
$$

where $Z \sim \mathcal{N}(0,1)$ and $r=2 p /(2 p+1)$.

Letting $\hat{c}_{\alpha, \delta}$ denote the lower endpoint of the one-sided CI corresponding to $\hat{L}_{\delta}$, the $C I$ $\left[\hat{c}_{\alpha, \delta}, \infty\right)$ has asymptotic coverage at least $1-\alpha$. If $\delta$ is chosen to minimax the $\beta$ quantile excess length, (i.e. $\left.\delta=z_{\beta}+z_{1-\alpha}\right)$, then, if each $\mathcal{Q}_{n}$ contains a distribution where the $u_{i} s$ are normal, any other one-sided $C I[\hat{c}, \infty)$ with asymptotic coverage at least $1-\alpha$ must satisfy the efficiency bound

$$
\liminf _{n \rightarrow \infty} \frac{\sup _{f \in \mathcal{F}, Q \in \mathcal{Q}_{n}} q_{f, Q, \beta}(L f-\hat{c})}{\sup _{f \in \mathcal{F}, Q \in \mathcal{Q}_{n}} q_{f, Q, \beta}\left(L f-\hat{c}_{\alpha, \delta}\right)} \geq 1 .
$$

In addition, we have the following bound on the asymptotic efficiency improvement at any $f \in \mathcal{F}_{R D T, p}(0):$

$$
\liminf _{n \rightarrow \infty} \frac{\sup _{Q \in \mathcal{Q}_{n}} q_{f, Q, \beta}(L f-\hat{c})}{\sup _{Q \in \mathcal{Q}_{n}} q_{f, Q, \beta}\left(L f-\hat{c}_{\alpha, \delta}\right)} \geq \frac{2^{r}}{1+r} .
$$

The proof of Theorem E.1 is given in Supplemental Appendix H. The asymptotic efficiency bounds correspond to those in Section 3 under (29) with $r=2 p /(2 p+1)$. 


\section{Appendix F Unknown Error Distribution}

The Gaussian regression model (1) makes the assumption of normal i.i.d. errors with a known variance conditional on the $x_{i}$ 's, which is often unrealistic. This section considers a model that relaxes these assumptions on the error distribution:

$$
y_{i}=f\left(x_{i}\right)+u_{i},\left\{u_{i}\right\}_{i=1}^{n} \sim Q, f \in \mathcal{F}, Q \in \mathcal{Q}_{n}
$$

where $\mathcal{Q}_{n}$ denotes the set of possible joint distributions of $\left\{u_{i}\right\}_{i=1}^{n}$ and, as before, $\left\{x_{i}\right\}_{i=1}^{n}$ is deterministic and $\mathcal{F}$ is a convex set. We derive feasible versions of the optimal CIs in Section 3 and show their asymptotic validity (uniformly over $\mathcal{F}, \mathcal{Q}_{n}$ ) and asymptotic efficiency. As we discuss below, our results hold even in cases where the limiting form of the optimal estimator is unknown or may not exist, and where currently available methods for showing asymptotic efficiency, such as equivalence with Gaussian white noise, break down.

Since the distribution of the data $\left\{y_{i}\right\}_{i=1}^{n}$ now depends on both $f$ and $Q$, we now index probability statements by both of these quantities: $P_{f, Q}$ denotes the distribution under $(f, Q)$ and similarly for $E_{f, Q}$. The coverage requirements and definitions of minimax performance criteria in Section 3 are the same, but with infima and suprema over functions $f$ now taken over both functions $f$ and error distributions $Q \in \mathcal{Q}_{n}$. We will also consider asymptotic results. We use the notation $Z_{n} \underset{\mathcal{F}, \mathcal{Q}_{n}}{\stackrel{d}{\rightarrow}} \mathcal{L}$ to mean that $Z_{n}$ converges in distribution to $\mathcal{L}$ uniformly over $f \in \mathcal{F}$ and $Q \in \mathcal{Q}_{n}$, and similarly for $\underset{\mathcal{F}, \mathcal{Q}_{n}}{\stackrel{p}{\rightarrow}}$.

If the variance function is unknown, the estimator $\hat{L}_{\delta}$ is infeasible. However, we can form an estimate based on an estimate of the variance function, or based on some candidate variance function. For a candidate variance function $\tilde{\sigma}^{2}(\cdot)$, let $K_{\tilde{\sigma}(\cdot), n} f=\left(f\left(x_{1}\right) / \tilde{\sigma}\left(x_{1}\right), \ldots\right.$, $\left.f\left(x_{n}\right) / \tilde{\sigma}\left(x_{n}\right)\right)^{\prime}$, and let $\omega_{\tilde{\sigma}(\cdot), n}(\delta)$ denote the modulus of continuity defined with this choice

of $K$. Let $\hat{L}_{\delta, \tilde{\sigma}(\cdot)}=\hat{L}_{\delta, \mathcal{F}, \mathcal{G}, \tilde{\sigma}(\cdot)}$ denote the estimator defined in (23) with this choice of $K$ and $Y=\left(y_{1} / \tilde{\sigma}\left(x_{1}\right), \ldots, y_{n} / \tilde{\sigma}\left(x_{n}\right)\right)^{\prime}$, and let $f_{\tilde{\sigma}(\cdot), \delta}^{*}$ and $g_{\tilde{\sigma}(\cdot), \delta}^{*}$ denote the least favorable functions used in forming this estimate. We assume throughout this section that $\mathcal{G} \subseteq \mathcal{F}$. More generally, we will consider affine estimators, which, in this setting, take the form

$$
\hat{L}=a_{n}+\sum_{i=1}^{n} w_{i, n} y_{i}
$$

where $a_{n}$ and $w_{i, n}$ are a sequence and triangular array respectively. For now, we assume that $a_{n}$ and $w_{i, n}$ are nonrandom, (which, in the case of the estimator $\hat{L}_{\delta, \tilde{\sigma}(\cdot)}$, requires that 
$\tilde{\sigma}(\cdot)$ and $\delta$ be nonrandom). We provide conditions that allow for random $a_{n}$ and $w_{i, n}$ after stating our result for nonrandom weights. For a class $\mathcal{G}$, the maximum and minimum bias are

$$
\overline{\operatorname{bias}}_{\mathcal{G}}(\hat{L})=\sup _{f \in \mathcal{G}}\left[a_{n}+\sum_{i=1}^{n} w_{i, n} f\left(x_{i}\right)-L f\right], \quad \underline{\operatorname{bias}}_{\mathcal{G}}(\hat{L})=\inf _{f \in \mathcal{G}}\left[a_{n}+\sum_{i=1}^{n} w_{i, n} f\left(x_{i}\right)-L f\right] .
$$

By the arguments used to derive the formula (24), we have

$$
\overline{\operatorname{bias}}_{\mathcal{F}}\left(\hat{L}_{\delta, \mathcal{F}, \mathcal{G}, \tilde{\sigma}(\cdot)}\right)=-\underline{\operatorname{bias}}_{\mathcal{G}}\left(\hat{L}_{\delta, \mathcal{F}, \mathcal{G}, \tilde{\sigma}(\cdot)}\right)=\frac{1}{2}\left(\omega_{n, \tilde{\sigma}(\cdot)}(\delta ; \mathcal{F}, \mathcal{G})-\delta \omega_{n, \tilde{\sigma}(\cdot)}^{\prime}(\delta ; \mathcal{F}, \mathcal{G})\right)
$$

This holds regardless of whether $\tilde{\sigma}(\cdot)$ is equal to the actual variance function of the $u_{i}$ 's. In our results below, we allow for infeasible estimators in which $a_{n}$ and $w_{i, n}$ depend on $Q$ (for example, when the unknown variance $\sigma_{Q}\left(x_{i}\right)=\operatorname{var}_{Q}\left(y_{i}\right)$ is used to compute the optimal weights), so that $\overline{\operatorname{bias}}_{\mathcal{G}}(\hat{L})$ and $\underline{\operatorname{bias}}_{\mathcal{G}}(\hat{L})$ may depend on $Q$. We leave this implicit in our notation.

Let $s_{n, Q}$ denote the (constant over $f$ ) standard deviation of $\hat{L}$ under $Q$ and suppose that the uniform central limit theorem

$$
\frac{\sum_{i=1}^{n} w_{i, n} u_{i}}{s_{n, Q}} \underset{\mathcal{F}, \mathcal{Q}_{n}}{\stackrel{d}{\longrightarrow}} \mathcal{N}(0,1)
$$

holds. To form a feasible CI, we will require an estimate $\widehat{\mathrm{se}}_{n}$ of $s_{n, Q}$ satisfying

$$
\frac{\widehat{\mathrm{se}}_{n}}{s_{n, Q}} \underset{\mathcal{F}, \mathcal{Q}_{n}}{\stackrel{p}{\rightarrow}} 1
$$

The following theorem shows that using $\widehat{\operatorname{se}}_{n}$ to form analogues of the CIs treated in Section 3 gives asymptotically valid CIs.

Theorem F.1. Let $\hat{L}$ be an estimator of the form (S12), and suppose that (S13) and (S14) hold. Let $\hat{c}=\hat{L}-\overline{\operatorname{bias}}_{\mathcal{F}}(\hat{L})-\widehat{s e}_{n} z_{1-\alpha}$, and let $b=\max \left\{\left|\overline{\text { bias }}_{\mathcal{F}}(\hat{L})\right|,\left|\underline{\text { bias }}_{\mathcal{F}}(\hat{L})\right|\right\}$. Then

$$
\liminf _{n \rightarrow \infty} \inf _{f \in \mathcal{F}, Q \in \mathcal{Q}_{n}} P_{f, Q}(L f \in[\hat{c}, \infty)) \geq 1-\alpha
$$

and

$$
\liminf _{n \rightarrow \infty} \inf _{f \in \mathcal{F}, Q \in \mathcal{Q}_{n}} P_{f, Q}\left(L f \in\left\{\hat{L} \pm \widehat{s e}_{n} \mathrm{cv}_{\alpha}\left(b / \widehat{s e}_{n}\right)\right\}\right) \geq 1-\alpha
$$


The worst-case $\beta$ th quantile excess length of the one-sided CI over $\mathcal{G}$ will satisfy

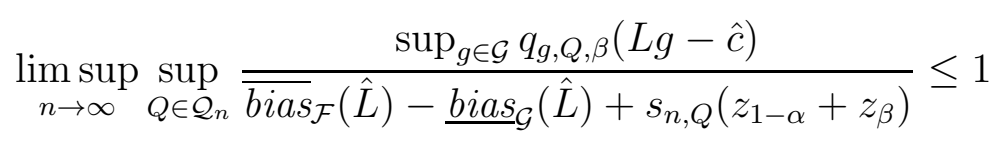

and the length of the two-sided CI will satisfy

$$
\frac{\mathrm{cv}_{\alpha}\left(b / \widehat{s e}_{n}\right) \widehat{s e}_{n}}{\mathrm{cv}_{\alpha}\left(b / s_{n, Q}\right) s_{n, Q}} \underset{\mathcal{F}, \mathcal{Q}_{n}}{\stackrel{p}{\rightarrow}} 1
$$

Suppose, in addition, that $\hat{L}=\hat{L}_{\delta, \mathcal{F}, \mathcal{G}, \tilde{\sigma}(\cdot)}$ with $\tilde{\sigma}(\cdot)=\sigma_{Q}(\cdot)$ where $\sigma_{Q}^{2}\left(x_{i}\right)=\operatorname{var}_{Q}\left(u_{i}\right)$ and, for each $n$, there exists a $Q_{n} \in \mathcal{Q}_{n}$ such that $\left\{u_{i}\right\}_{i=1}^{n}$ are independent and normal under $Q_{n}$. Then no one-sided CI satisfying (S15) can satisfy (S17) with the constant 1 replaced by a strictly smaller constant on the right-hand side.

Proof. Let $Z_{n}=\sum_{i=1}^{n} w_{i, n} u_{i} / \widehat{\mathrm{se}}_{n}$, and let $Z$ denote a standard normal random variable. To show asymptotic coverage of the one-sided CI, note that

$$
P_{f, Q}(L f \in[\hat{c}, \infty))=P_{f, Q}\left(\widehat{\operatorname{se}}_{n} z_{1-\alpha} \geq \hat{L}-L f-\overline{\operatorname{bias}}_{\mathcal{F}}(\hat{L})\right) \geq P_{f, Q}\left(z_{1-\alpha} \geq Z_{n}\right)
$$

using the fact that $\overline{\operatorname{bias}}_{\mathcal{F}}(\hat{L})+\sum_{i=1}^{n} w_{i, n} u_{i} \geq \hat{L}-L f$ for all $f \in \mathcal{F}$ by the definition of $\overline{\operatorname{bias}}_{\mathcal{F}}$. The right-hand side converges to $1-\alpha$ uniformly over $f \in \mathcal{F}$ and $Q \in \mathcal{Q}_{n}$ by (S13) and (S14). For the two-sided CI, first note that

$$
\left|\frac{\mathrm{cv}_{\alpha}\left(b / \widehat{\mathrm{e}}_{n}\right) \widehat{\mathrm{se}}_{n}}{\mathrm{cv}_{\alpha}\left(b / s_{n, Q}\right) s_{n, Q}}-1\right|=\left|\frac{\mathrm{cv}_{\alpha}\left(b / \widehat{\mathrm{se}}_{n}\right)-\mathrm{cv}_{\alpha}\left(b / s_{n, Q}\right)+\mathrm{cv}_{\alpha}\left(b / s_{n, Q}\right)\left(1-s_{n, Q} / \widehat{\mathrm{se}}_{n}\right)}{\mathrm{cv}_{\alpha}\left(b / s_{n, Q}\right)\left(s_{n, Q} / \widehat{\mathrm{se}}_{n}\right)}\right|
$$

which converges to zero uniformly over $f \in \mathcal{F}, Q \in \mathcal{Q}_{n}$ since $\mathrm{cv}_{\alpha}(t)$ is bounded from below and uniformly continuous with respect to $t$. Thus, $\frac{\mathrm{cv} \alpha_{\alpha}\left(b / \widehat{\mathrm{se}}_{n}\right) \widehat{\mathrm{se}}_{n}}{\mathrm{cv} \alpha_{\alpha}\left(b / s_{n, Q}\right) s_{n, Q}} \underset{\mathcal{F}, \mathcal{Q}_{n}}{\stackrel{p}{\rightarrow}} 1$ as claimed. To show coverage of the two-sided CI, note that

$$
P_{f, Q}\left(L f \in\left\{\hat{L} \pm \mathrm{cv}_{\alpha}\left(b / \widehat{\mathrm{se}}_{n}\right) \widehat{\mathrm{se}}_{n}\right\}\right)=P_{f, Q}\left(\left|\tilde{Z}_{n}+r\right| \leq \mathrm{cv}_{\alpha}\left(b / s_{n, Q}\right) \cdot c_{n}\right)
$$

where $c_{n}=\frac{\mathrm{cv}_{\alpha}\left(b / \widehat{\mathrm{se}}_{n}\right) \widehat{\mathrm{s}}_{n}}{\operatorname{cv} \alpha_{\alpha}\left(b / s_{n, Q}\right) s_{n, Q}}, \tilde{Z}_{n}=\sum_{i=1}^{n} w_{i, n} u_{i} / s_{n, Q}$ and $r=\left(a_{n}+\sum_{i=1}^{n} w_{i, n} f\left(x_{i}\right)-L f\right) / s_{n, Q}$. By (S13) and the fact that $c_{n} \underset{\mathcal{F}, \mathcal{Q}_{n}}{\stackrel{p}{\rightarrow}} 1$, this is equal to $P_{f, Q}\left(|Z+r| \leq \mathrm{cv}_{\alpha}\left(b / s_{n, Q}\right)\right.$ ) (where $Z \sim \mathcal{N}(0,1)$ ) plus a term that converges to zero uniformly over $f, Q$ (this can be seen by using the fact that convergence in distribution to a continuous distribution implies uniform convergence of the cdfs; see Lemma 2.11 in van der Vaart 1998). Since $|r| \leq b / s_{n, Q}$, (S16) 
follows.

To show (S17), note that,

$$
\begin{aligned}
L g-\hat{c}=L g-a_{n}-\sum_{i=1}^{n} w_{i, n} g\left(x_{i}\right)-\widehat{\operatorname{se}}_{n} Z_{n}+\overline{\operatorname{bias}}_{\mathcal{F}}(\hat{L})+\widehat{\operatorname{se}}_{n} z_{1-\alpha} \\
\quad \leq \overline{\operatorname{bias}}_{\mathcal{F}}(\hat{L})-\underline{\operatorname{bias}_{\mathcal{G}}(\hat{L})+\widehat{\operatorname{se}}_{n}\left(z_{1-\alpha}-Z_{n}\right)}
\end{aligned}
$$

for any $g \in \mathcal{G}$. Thus,

$$
\begin{aligned}
\frac{L g-\hat{c}}{\overline{\overline{\operatorname{bias}}}_{\mathcal{F}}(\hat{L})-\underline{\operatorname{bias}}_{\mathcal{G}}(\hat{L})+s_{n, Q}\left(z_{1-\alpha}+z_{\beta}\right)}-1 & \leq \frac{\widehat{\operatorname{se}}_{n}\left(z_{1-\alpha}-Z_{n}\right)-s_{n, Q}\left(z_{1-\alpha}+z_{\beta}\right)}{\overline{\operatorname{bias}}_{\mathcal{F}}(\hat{L})-\underline{\operatorname{bias}}_{\mathcal{G}}(\hat{L})+s_{n, Q}\left(z_{1-\alpha}+z_{\beta}\right)} \\
& =\frac{\left(\widehat{\operatorname{se}}_{n} / s_{n, Q}\right) \cdot\left(z_{1-\alpha}-Z_{n}\right)-\left(z_{1-\alpha}+z_{\beta}\right)}{\left[\overline{\operatorname{bias}}_{\mathcal{F}}(\hat{L})-\underline{\operatorname{bias}}_{\mathcal{G}}(\hat{L})\right] / s_{n, Q}+\left(z_{1-\alpha}+z_{\beta}\right)} .
\end{aligned}
$$

The $\beta$ quantile of the above display converges to 0 uniformly over $f \in \mathcal{F}$ and $Q \in \mathcal{Q}_{n}$, which gives the result.

For the last statement, let $[\tilde{c}, \infty)$ be a sequence of CIs with asymptotic coverage $1-\alpha$. Let $Q_{n}$ be the distribution from the conditions in the theorem, in which the $u_{i}$ 's are independent and normal. Then, by Theorem 3.1,

$$
\sup _{g \in \mathcal{F}} q_{f, Q_{n}, \beta}(\tilde{c}-L g) \geq \omega_{\sigma_{Q_{n}}(\cdot), n}\left(\tilde{\delta}_{n}\right)
$$

where $\tilde{\delta}_{n}=z_{1-\alpha_{n}}+z_{\beta}$ and $1-\alpha_{n}$ is the coverage of $[\tilde{c}, \infty)$ over $\mathcal{F}, \mathcal{Q}_{n}$. When $\hat{L}=\hat{L}_{\delta, \mathcal{F}, \mathcal{G}, \sigma_{Q}(\cdot)}$, the denominator in (S17) for $Q=Q_{n}$ is equal to $\omega_{\sigma_{Q_{n}}(\cdot), n}\left(z_{1-\alpha}+z_{\beta}\right)$, which gives

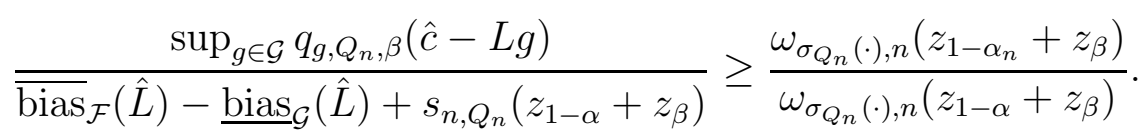

If $\alpha_{n} \leq \alpha$, then $z_{1-\alpha_{n}}+z_{\beta} \geq z_{1-\alpha}-z_{\beta}$ so that the above display is greater than one by monotonicity of the modulus. If not, then by concavity, $\omega_{\sigma_{Q_{n}}(\cdot), n}\left(z_{1-\alpha_{n}}+z_{\beta}\right) \geq\left[\omega_{\sigma_{Q_{n}}(\cdot), n}\left(z_{1-\alpha}+\right.\right.$ $\left.\left.z_{\beta}\right) /\left(z_{1-\alpha}+z_{\beta}\right)\right] \cdot\left(z_{1-\alpha_{n}}+z_{\beta}\right)$, so the above display is bounded from below by $\left(z_{1-\alpha_{n}}+\right.$ $\left.z_{\beta}\right) /\left(z_{1-\alpha}+z_{\beta}\right)$, and the lim inf of this is at least one by the coverage requirement.

The efficiency bounds in Theorem F.1 use the assumption that the class of possible distributions contains a normal law, as is often done in the literature on efficiency in nonparametric settings (see, e.g., Fan, 1993, pp. 205-206). We leave the topic of relaxing this assumption for future research. 
Theorem F.1 requires that a known candidate variance function $\tilde{\sigma}(\cdot)$ and a known $\delta$ be used when forming CIs based on the estimate $\hat{L}_{\delta}$. However, the theorem does not require that the candidate variance function be correct in order to get asymptotic coverage, so long as the standard error $\widehat{\mathrm{se}}_{n}$ is consistent. If it turns out that $\tilde{\sigma}(\cdot)$ is indeed the correct variance function, then it follows from the last part of the theorem that the resulting CI is efficient. In the special case where $\mathcal{F}$ imposes a (otherwise unconstrained) linear model, this corresponds to the common practice of using ordinary least squares with heteroskedasticity robust standard errors.

In some cases, one will want to use a data dependent $\tilde{\sigma}(\cdot)$ and $\delta$ in order to get efficient estimates with unknown variance. The asymptotic coverage and efficiency of the resulting CI can then be derived by showing equivalence with the infeasible estimator $\hat{L}_{\delta^{*}, \mathcal{F}, \mathcal{G}, \sigma_{Q}(\cdot) \text {, where }}$ $\delta^{*}$ is chosen according to the desired performance criterion. The following theorem gives conditions for this asymptotic equivalence. We verify them for our regression discontinuity example in Supplemental Appendix H.

Theorem F.2. Suppose that $\hat{L}$ and $\widehat{s e}_{n}$ satisfy (S13) and (S14). Let $\tilde{L}$ and $\widetilde{s e}_{n}$ be another estimator and standard error, and let $\widetilde{\overline{b i a s}}_{n}$ and $\widetilde{\text { bias }}_{n}$ be (possibly data dependent) worst-case bias formulas for $\tilde{L}$ under $\mathcal{F}$. Suppose that

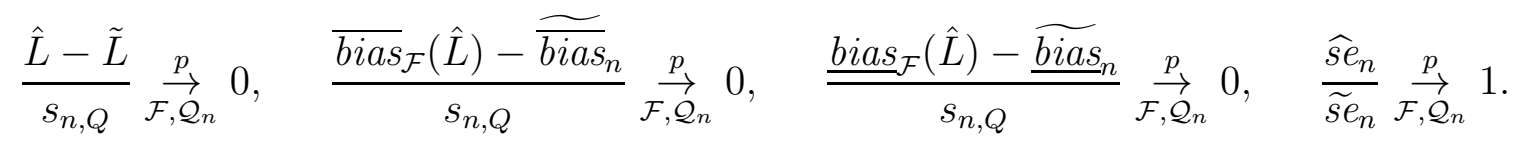

Let $\tilde{c}=\tilde{L}-\widetilde{\text { bias }_{n}}-\widetilde{s e}_{n} z_{1-\alpha}$, and let $\tilde{b}=\max \left\{\left|\widetilde{\text { bias }_{n} \mid}\right| \widetilde{\text { bias }_{n}} \mid\right\}$. Then (S15) and (S16) hold with $\hat{c}$ replaced by $\tilde{c}, \hat{L}$ replaced by $\tilde{L}$, b replaced by $\tilde{b}$ and $\widehat{s}_{n}$ replaced by $\widetilde{s e}_{n}$. Furthermore, the performance of the CIs is asymptotically equivalent in the sense that

$$
\frac{\sup _{Q \in \mathcal{Q}_{n}} \sup _{g \in \mathcal{G}} q_{g, Q, \beta}(\tilde{c}-L g)}{\sup _{Q \in \mathcal{Q}_{n}} \sup _{g \in \mathcal{G}} q_{g, Q, \beta}(\hat{c}-L g)} \rightarrow 1 \text { and } \frac{\mathrm{cv}_{\alpha}\left(b / \widehat{s e}_{n}\right) \widehat{s e}_{n}}{\mathrm{cv}_{\alpha}\left(\tilde{b} / \widetilde{s e}_{n}\right) \widetilde{s e}_{n}} \underset{\mathcal{F}, \mathcal{Q}_{n}}{\stackrel{p}{\rightarrow}} 1
$$

Proof. By the conditions of the theorem, we have, for some $c_{n}$ that converges in probability to zero uniformly over $\mathcal{F}, \mathcal{Q}_{n}$,

$$
\begin{aligned}
\tilde{c}-L f=\tilde{L}-L f-\widetilde{\widetilde{\operatorname{bias}}_{n}}-\widetilde{\operatorname{se}}_{n} z_{1-\alpha}=\hat{L}-L f-\overline{\operatorname{bias}}_{\mathcal{F}}(\hat{L})-s_{n, Q} z_{1-\alpha}+c_{n} s_{n, Q} \\
\leq \sum_{i=1}^{n} w_{i, n} u_{i}-s_{n, Q} z_{1-\alpha}+c_{n} s_{n, Q} .
\end{aligned}
$$


Thus,

$$
P_{f, Q}(L f \in[\tilde{c}, \infty))=P_{f, Q}(0 \geq \tilde{c}-L f) \geq P_{f, Q}\left(0 \geq \frac{\sum_{i=1}^{n} w_{i, n} u_{i}}{s_{n, Q}}-z_{1-\alpha}+c_{n}\right)
$$

which converges to $1-\alpha$ uniformly over $\mathcal{F}, \mathcal{Q}_{n}$. By Theorem F.1, $\sup _{g \in \mathcal{G}} q_{g, Q, \beta}(\hat{c}-L g)$ is bounded from below by a constant times $s_{n, Q}$. Thus, $\left|\frac{\sup _{Q \in \mathcal{Q}_{n}} \sup _{g \in \mathcal{G}} q_{g, Q, \beta}(\tilde{c}-L g)}{\sup _{Q \in \mathcal{Q}_{n}} \sup _{g \in \mathcal{G}} q_{g, Q, \beta}(\hat{c}-L g)}-1\right|$ is bounded from above by a constant times

$$
\sup _{Q \in \mathcal{Q}_{n}} \sup _{g \in \mathcal{G}}\left|\frac{q_{g, Q, \beta}(\tilde{c}-L g)-q_{g, Q, \beta}(\hat{c}-L g)}{s_{n, Q}}\right|=\sup _{Q \in \mathcal{Q}_{n}} \sup _{g \in \mathcal{G}}\left|q_{g, Q, \beta}\left(\tilde{c} / s_{n, Q}\right)-q_{g, Q, \beta}\left(\hat{c} / s_{n, Q}\right)\right|
$$

which converges to zero since $(\tilde{c}-\hat{c}) / s_{n, Q} \underset{\mathcal{F}, \mathcal{Q}_{n}}{\stackrel{p}{\rightarrow}} 0$.

The claim that $\frac{\mathrm{cv}_{\alpha}\left(b / \widehat{\mathrm{se}}_{n}\right) \widehat{\mathrm{se}}_{n}}{\mathrm{cv}_{\alpha}\left(\tilde{b} / \widetilde{\mathrm{se}}_{n}\right) \widetilde{\mathrm{se}}_{n}} \underset{\mathcal{F}, \mathcal{Q}_{n}}{\stackrel{p}{\rightarrow}} 1$ follows using similar arguments to the proof of Theorem F.1. To show coverage of the two-sided CI, note that

$$
P_{f, Q}\left(L f \in\left\{\tilde{L} \pm \mathrm{cv}_{\alpha}\left(\tilde{b} / \widetilde{\mathrm{se}}_{n}\right) \widetilde{\mathrm{se}}_{n}\right\}\right)=P_{f, Q}\left(\frac{|\tilde{L}-L f|}{s_{n, Q}} \leq \mathrm{cv}_{\alpha}\left(b / s_{n, Q}\right) \cdot c_{n}\right)
$$

where $c_{n}=\frac{\mathrm{cv}_{\alpha}\left(\tilde{b} / \widetilde{\mathrm{se}}_{n}\right) \widetilde{\mathrm{se}}_{n}}{\mathrm{cv}_{\alpha}\left(b / s_{n, Q}\right) s_{n, Q}} \underset{\mathcal{F}, \mathcal{Q}_{n}}{\stackrel{p}{\rightarrow}} 1$. Since $\frac{|\tilde{L}-L f|}{s_{n, Q}}=\left|V_{n}+r\right|$ where $r=\left(a_{n}+\sum_{i=1}^{n} w_{i, n} f\left(x_{i}\right)-\right.$ $L f) / s_{n, Q}$ and $V_{n}=\sum_{i=1}^{n} w_{i, n} u_{i} / s_{n, Q}+(\tilde{L}-\hat{L}) / s_{n, Q} \underset{\mathcal{F}, \mathcal{Q}_{n}}{\stackrel{d}{\rightarrow}} \mathcal{N}(0,1)$, the result follows from arguments in the proof of Theorem F.1.

The results above give high-level conditions that can be applied to a wide range of estimators and CIs. We now introduce an estimator and standard error formula that give asymptotic coverage for essentially arbitrary functionals $L$ under generic low level conditions on $\mathcal{F}$ and the $x_{i}$ 's. The estimator is based on a nonrandom guess for the variance function and, if this guess is correct up to scale (e.g. if the researcher correctly guesses that the errors are homoskedastic), the one-sided CI based on this estimator will be asymptotically optimal for some quantile of excess length.

Let $\tilde{\sigma}(\cdot)$ be some nonrandom guess for the variance function bounded away from 0 and $\infty$, and let $\delta>0$ be a deterministic constant specified by the researcher. Let $\hat{f}$ be an estimator of $f$. The variance of $\hat{L}_{\delta, \tilde{\sigma}(\cdot)}$ under some $Q \in \mathcal{Q}_{n}$ is equal to

$$
\operatorname{var}_{Q}\left(\hat{L}_{\delta, \tilde{\sigma}(\cdot), n}\right)=\left(\frac{\omega_{\tilde{\sigma}(\cdot), n}^{\prime}(\delta)}{\delta}\right)^{2} \sum_{i=1}^{n} \frac{\left(g_{\tilde{\sigma}(\cdot), \delta}^{*}\left(x_{i}\right)-f_{\tilde{\sigma}(\cdot), \delta}^{*}\left(x_{i}\right)\right)^{2} \sigma_{Q}^{2}\left(x_{i}\right)}{\tilde{\sigma}^{4}\left(x_{i}\right)}
$$


We consider the estimate

$$
\widehat{\operatorname{se}}_{\delta, \tilde{\sigma}(\cdot), n}^{2}=\left(\frac{\omega_{\tilde{\sigma}(\cdot), n}^{\prime}(\delta)}{\delta}\right)^{2} \sum_{i=1}^{n} \frac{\left(g_{\tilde{\sigma}(\cdot), \delta}^{*}\left(x_{i}\right)-f_{\tilde{\sigma}(\cdot), \delta}^{*}\left(x_{i}\right)\right)^{2}\left(y_{i}-\hat{f}\left(x_{i}\right)\right)^{2}}{\tilde{\sigma}^{4}\left(x_{i}\right)}
$$

Suppose that $f: \mathcal{X} \rightarrow \mathbb{R}$ where $\mathcal{X}$ is a metric space with metric $d_{X}$ such that the functions $f_{\tilde{\sigma}(\cdot), \delta}^{*}$ and $g_{\tilde{\sigma}(\cdot), \delta}^{*}$ satisfy the uniform continuity condition

$$
\sup _{n} \sup _{x, x^{\prime}: d_{X}\left(x, x^{\prime}\right) \leq \eta} \max \left\{\left|f_{\tilde{\sigma}(\cdot), \delta}^{*}(x)-f_{\tilde{\sigma}(\cdot), \delta}^{*}\left(x^{\prime}\right)\right|,\left|g_{\tilde{\sigma}(\cdot), \delta}^{*}(x)-g_{\tilde{\sigma}(\cdot), \delta}^{*}\left(x^{\prime}\right)\right|\right\} \leq \bar{g}(\eta),
$$

where $\lim _{\eta \rightarrow 0} \bar{g}(\eta)=0$ and, for all $\eta>0$,

$$
\min _{1 \leq i \leq n} \sum_{j=1}^{n} I\left(d_{X}\left(x_{j}, x_{i}\right) \leq \eta\right) \rightarrow \infty
$$

We also assume that the estimator $\hat{f}$ used to form the variance estimate satisfies the uniform convergence condition

$$
\max _{1 \leq i \leq n}\left|\hat{f}\left(x_{i}\right)-f\left(x_{i}\right)\right| \underset{\mathcal{F}, \mathcal{Q}_{n}}{\stackrel{p}{\rightarrow}} 0 .
$$

Finally, we impose conditions on the moments of the error distribution. Suppose that there exist $K$ and $\eta>0$ such that, for all $n, Q \in \mathcal{Q}_{n}$, the errors $\left\{u_{i}\right\}_{i=1}^{n}$ are independent with, for each $i$,

$$
1 / K \leq \sigma_{Q}^{2}\left(x_{i}\right) \leq K \text { and } E_{Q}\left|u_{i}\right|^{2+\eta} \leq K
$$

In cases where function class $\mathcal{F}$ imposes smoothness on $f,(\mathrm{~S} 18)$ will often follow directly from the definition of $\mathcal{F}$. For example, it holds for the Lipschitz class $\left\{f:\left|f(x)-f\left(x^{\prime}\right)\right| \leq\right.$ $\left.C d_{X}\left(x, x^{\prime}\right)\right\}$. The condition (S19) will hold with probability one if the $x_{i}$ 's are sampled from a distribution with density bounded away from zero on a sufficiently regular bounded support. The condition (S20) will hold under regularity conditions for a variety of choices of $\hat{f}$. It is worth noting that smoothness assumptions on $\mathcal{F}$ needed for this assumption are typically weaker than those needed for asymptotic equivalence with Gaussian white noise. For example, if $\mathcal{X}=\mathbb{R}^{k}$ with the Euclidean norm, (S18) will hold automatically for Hölder classes with exponent less than or equal to 1, while equivalence with Gaussian white noise requires that the exponent be greater than $k / 2$ (see Brown and Zhang, 1998). Furthermore, we do not require any explicit characterization of the limiting form of the optimal CI. In particular, we do not require that the weights for the optimal estimator converge to a limiting 
optimal kernel or efficient influence function.

The condition (S21) is used to verify a Lindeberg condition for the central limit theorem used to obtain (S13), which we do in the next lemma.

Lemma F.1. Let $Z_{n, i}$ be a triangular array of independent random variables and let $a_{n, j}$, $1 \leq j \leq n$ be a triangular array of constants. Suppose that there exist constants $K$ and $\eta>0$ such that, for all $i$,

$$
1 / K \leq \sigma_{n, i}^{2} \leq K \text { and } E\left|Z_{n, i}\right|^{2+\eta} \leq K
$$

where $\sigma_{n, i}^{2}=E Z_{n, i}^{2}$, and that

$$
\lim _{n \rightarrow \infty} \frac{\max _{1 \leq j \leq n} a_{n, j}^{2}}{\sum_{j=1}^{n} a_{n, j}^{2}}=0 .
$$

Then

$$
\frac{\sum_{i=1}^{n} a_{n, i} Z_{n, i}}{\sqrt{\sum_{i=1}^{n} a_{n, i}^{2} \sigma_{n, i}^{2}}} \stackrel{d}{\rightarrow} \mathcal{N}(0,1) .
$$

Proof. We verify the conditions of the Lindeberg-Feller theorem as stated on p. 116 in Durrett (1996), with $X_{n, i}=a_{n, i} Z_{n, i} / \sqrt{\sum_{j=1}^{n} a_{n, j}^{2} \sigma_{j}^{2}}$. To verify the Lindeberg condition, note that

$$
\begin{aligned}
& \sum_{i=1}^{n} E\left(\left|X_{n, m}\right|^{2} 1\left(\left|X_{n, m}\right|>\varepsilon\right)\right)=\frac{\sum_{i=1}^{n} E\left[\left|a_{n, i} Z_{n, i}\right|^{2} I\left(\left|a_{n, i} Z_{n, i}\right|>\varepsilon \sqrt{\sum_{j=1}^{n} a_{n, j}^{2} \sigma_{j}^{2}}\right)\right]}{\sum_{i=1}^{n} a_{n, i}^{2} \sigma_{n, i}^{2}} \\
& \leq \frac{\sum_{i=1}^{n} E\left(\left|a_{n, i} Z_{n, i}\right|^{2+\eta}\right)}{\varepsilon^{\eta}\left(\sum_{i=1}^{n} a_{n, i}^{2} \sigma_{n, i}^{2}\right)^{1+\eta / 2}} \leq \frac{K^{2+\eta / 2}}{\varepsilon^{\eta}} \frac{\sum_{i=1}^{n}\left|a_{n, i}\right|^{2+\eta}}{\left(\sum_{i=1}^{n} a_{n, i}^{2}\right)^{1+\eta / 2}} \leq \frac{K^{2+\eta / 2}}{\varepsilon^{\eta}}\left(\frac{\max _{1 \leq i \leq n} a_{n, i}^{2}}{\sum_{i=1}^{n} a_{n, i}^{2}}\right)^{1+\eta / 2} .
\end{aligned}
$$

This converges to zero under the conditions of the lemma.

Theorem F.3. Let $\hat{L}_{\delta, \tilde{\sigma}(\cdot)}$ and $\widehat{s e}_{\delta, \tilde{\sigma}(\cdot), n}^{2}$ be defined above. Suppose that, for each $n, f_{\tilde{\sigma}(\cdot), \delta}^{*}$, $g_{\tilde{\sigma}(\cdot), \delta}^{*}$ achieve the modulus under $\tilde{\sigma}(\cdot)$ with $\left\|K_{\tilde{\sigma}(\cdot), n}\left(g_{\tilde{\sigma}(\cdot), \delta}^{*}-f_{\tilde{\sigma}(\cdot), \delta}^{*}\right)\right\|=\delta$, and that (S18) and (S19) hold. Suppose the errors satisfy (S21) and are independent over $i$ for all $n$ and $Q \in \mathcal{Q}_{n}$. Then (S13) holds. If, in addition, the estimator $\hat{f}$ satisfies (S20), then (S14) holds with $\widehat{s e}_{n}$ given by $\widehat{s e}_{\delta, \tilde{\sigma}(\cdot), n}$.

Proof. Condition (S13) will follow by applying Lemma F.1 to show convergence under arbi- 
trary sequences $Q_{n} \in \mathcal{Q}_{n}$ so long as

$$
\frac{\max _{1 \leq i \leq n}\left(g_{\tilde{\sigma}(\cdot), \delta}^{*}\left(x_{i}\right)-f_{\tilde{\sigma}(\cdot), \delta}^{*}\left(x_{i}\right)\right)^{2} / \tilde{\sigma}\left(x_{i}\right)^{4}}{\sum_{i=1}^{n}\left(f_{\tilde{\sigma}(\cdot), \delta}^{*}\left(x_{i}\right)-g_{\tilde{\sigma}(\cdot), \delta}^{*}\left(x_{i}\right)\right)^{2} / \tilde{\sigma}\left(x_{i}\right)^{4}} \rightarrow 0 .
$$

Since the denominator is bounded from below by $\delta^{2} / \max _{1 \leq i \leq n} \tilde{\sigma}^{2}\left(x_{i}\right)$, and $\tilde{\sigma}^{2}\left(x_{i}\right)$ is bounded away from 0 and $\infty$ over $i$, it suffices to show that $\max _{1 \leq i \leq n}\left(g_{\tilde{\sigma}(\cdot), \delta}^{*}\left(x_{i}\right)-f_{\tilde{\sigma}(\cdot), \delta}^{*}\left(x_{i}\right)\right)^{2} \rightarrow 0$. To this end, suppose, to the contrary, that there exists some $c>0$ such that $\max _{1 \leq i \leq n}\left(g_{\tilde{\sigma}(\cdot), \delta}^{*}\left(x_{i}\right)\right.$ $\left.-f_{\tilde{\sigma}(\cdot), \delta}^{*}\left(x_{i}\right)\right)^{2}>c^{2}$ infinitely often. Let $\eta$ be small enough so that $\bar{g}(\eta) \leq c / 4$. Then, for $n$ such that this holds and $k_{n}$ achieving this maximum,

$$
\sum_{i=1}^{n}\left(g_{\tilde{\sigma}(\cdot), \delta}^{*}\left(x_{i}\right)-f_{\tilde{\sigma}(\cdot), \delta}^{*}\left(x_{i}\right)\right)^{2} \geq \sum_{i=1}^{n}(c-c / 2)^{2} 1\left(d_{X}\left(x_{i}, x_{k_{n}}\right) \leq \eta\right) \rightarrow \infty
$$

But this is a contradiction since $\sum_{i=1}^{n}\left(g_{\tilde{\sigma}(\cdot), \delta}^{*}\left(x_{i}\right)-f_{\tilde{\sigma}(\cdot), \delta}^{*}\left(x_{i}\right)\right)^{2}$ is bounded by a constant times $\sum_{i=1}^{n}\left(g_{\tilde{\sigma}(\cdot), \delta}^{*}\left(x_{i}\right)-f_{\tilde{\sigma}(\cdot), \delta}^{*}\left(x_{i}\right)\right)^{2} / \tilde{\sigma}^{2}\left(x_{i}\right)=\delta^{2}$.

To show convergence of $\widehat{\operatorname{se}}_{\delta, \tilde{\sigma}(\cdot), n}^{2} / \operatorname{var}_{Q}\left(\hat{L}_{\delta, \tilde{\sigma}(\cdot)}\right)$, note that

$$
\frac{\widehat{\operatorname{se}}_{\delta, \tilde{\sigma}(\cdot), n}^{2}}{\operatorname{var}_{Q}\left(\hat{L}_{\delta, \tilde{\sigma}(\cdot)}\right)}-1=\frac{\sum_{i=1}^{n} a_{n, i}\left[\left(y_{i}-\hat{f}\left(x_{i}\right)\right)^{2}-\sigma_{Q}^{2}\left(x_{i}\right)\right]}{\sum_{i=1}^{n} a_{n, i} \sigma_{Q}^{2}\left(x_{i}\right)}
$$

where $a_{n, i}=\frac{\left(g_{\tilde{\sigma}(\cdot), \delta}^{*}\left(x_{i}\right)-f_{\tilde{\sigma}(\cdot), \delta}^{*}\left(x_{i}\right)\right)^{2}}{\tilde{\sigma}^{4}\left(x_{i}\right)}$. Since the denominator is bounded from below by a constant times $\sum_{i=1}^{n} a_{n, i} \tilde{\sigma}^{2}\left(x_{i}\right)=\delta^{2}$, it suffices to show that the numerator, which can be written as

$$
\sum_{i=1}^{n} a_{n, i}\left[u_{i}^{2}-\sigma_{Q}\left(x_{i}\right)^{2}\right]+\sum_{i=1}^{n} a_{n, i}\left(f\left(x_{i}\right)-\hat{f}\left(x_{i}\right)\right)^{2}+2 \sum_{i=1}^{n} a_{n, i} u_{i}\left(f\left(x_{i}\right)-\hat{f}\left(x_{i}\right)\right),
$$

converges in probability to zero uniformly over $f$ and $Q$. The second term is bounded by a constant times $\max _{1 \leq i \leq n}\left(f\left(x_{i}\right)-\hat{f}\left(x_{i}\right)\right)^{2} \sum_{i=1}^{n} a_{n, i} \tilde{\sigma}^{2}\left(x_{i}\right)=\max _{1 \leq i \leq n}\left(f\left(x_{i}\right)-\hat{f}\left(x_{i}\right)\right)^{2} \delta^{2}$, which converges in probability to zero uniformly over $f$ and $Q$ by assumption. Similarly, the last term is bounded by $\max _{1 \leq i \leq n}\left|f\left(x_{i}\right)-\hat{f}\left(x_{i}\right)\right|$ times $2 \sum_{i=1}^{n} a_{n, i}\left|u_{i}\right|$, and the expectation of the latter term is bounded uniformly over $\mathcal{F}$ and $\mathcal{Q}$. Thus, the last term converges in probability to zero uniformly over $f$ and $Q$ as well. For the first term in this display, an inequality of von Bahr and Esseen (1965) shows that the expectation of the absolute $1+\eta / 2$ moment of 
this term is bounded by a constant times

$$
\sum_{i=1}^{n} a_{n, i}^{1+\eta / 2} E_{Q}\left|u_{i}^{2}-\sigma_{Q}\left(x_{i}\right)^{2}\right|^{1+\eta / 2} \leq\left(\max _{1 \leq i \leq n} a_{n, i}^{\eta / 2}\right) \max _{1 \leq i \leq n} E_{Q}\left|\varepsilon_{i}^{2}-\sigma_{Q}^{2}\left(x_{i}\right)\right|^{1+\eta / 2} \sum_{i=1}^{n} a_{n, i}
$$

which converges to zero since $\max _{1 \leq i \leq n} a_{n, i} \rightarrow 0$ as shown earlier in the proof and $\sum_{i=1}^{n} a_{n, i}$ is bounded by a constant times $\sum_{i=1}^{n} a_{n, i} \tilde{\sigma}^{2}\left(x_{i}\right)=\delta^{2}$.

If the variance function used by the researcher is correct up to scale (for example, if the variance function is known to be constant), the one-sided confidence intervals in (F.3) will be asymptotically optimal for some level $\beta$, which depends on $\delta$ and the magnitude of the true error variance relative to the one used by the researcher. We record this as a corollary.

Corollary F.1. If, in addition to the conditions in Theorem F.3, $\sigma_{Q}^{2}(x)=\sigma^{2} \cdot \tilde{\sigma}^{2}(x)$ for all $n$ and $Q \in \mathcal{Q}_{n}$, then, letting $\beta=\Phi\left(\delta / \sigma-z_{1-\alpha}\right)$, no CI satisfying (S15) can satisfy S17 with the constant 1 replaced by a strictly smaller constant on the right-hand side.

\section{Appendix G Asymptotics for the Modulus and Effi- ciency Bounds}

As discussed in Section 3, asymptotic relative efficiency comparisons can often be performed by calculating the limit of the scaled modulus. Here, we state some lemmas that can be used to obtain asymptotic efficiency bounds and limiting behavior of the value of $\delta$ that optimizes a particular performance criterion. We use these results in the proof of Theorem E.1 in Supplemental Appendix H.

Before stating these results, we recall the characterization of minimax affine performance given in Donoho (1994). To describe the results, first consider the normal model $Z \sim \mathcal{N}(\mu, 1)$ where $\mu \in[-\tau, \tau]$. The minimax affine mean squared error for this problem is

$$
\rho_{A}(\tau)=\min _{\delta(Y) \text { affine }} \max _{\mu \in[-\tau, \tau]} E_{\mu}(\delta(Y)-\mu)^{2}
$$

The solution is achieved by shrinking $Y$ toward 0 , namely $\delta(Y)=c_{\rho}(\tau) Y$, with $c_{\rho}(\tau)=$ $\tau^{2} /\left(1+\tau^{2}\right)$, which gives $\rho_{A}(\tau)=\tau^{2} /\left(1+\tau^{2}\right)$. The length of the smallest fixed-length affine 
$100 \cdot(1-\alpha) \%$ confidence interval is

$$
\chi_{A, \alpha}(\tau)=\min \left\{\chi: \text { there exists } \delta(Y) \text { affine s.t. } \inf _{\mu \in[-\tau, \tau]} P_{\mu}(|\delta(Y)-\mu| \leq \chi) \geq 1-\alpha\right\} .
$$

The solution is achieved at some $\delta(Y)=c_{\chi}(\tau) Y$, and it is characterized in Drees (1999).

Using these definitions, the minimax affine root MSE is given by

$$
\sup _{\delta>0} \frac{\omega(\delta)}{\delta} \sqrt{\rho_{A}\left(\frac{\delta}{2 \sigma}\right)} \sigma
$$

and the MSE optimal estimate is given by $\hat{L}_{\delta, \chi}$ where $\chi$ maximizes the above display. Similarly, the optimal fixed-length affine CI has half-length

$$
\sup _{\delta>0} \frac{\omega(\delta)}{\delta} \chi_{A, \alpha}\left(\frac{\delta}{2 \sigma}\right) \sigma
$$

and is centered at $\hat{L}_{\delta_{\chi}}$ where $\delta_{\chi}$ maximizes the above display (it follows from our results and those of Donoho 1994 that this leads to the same value of $\delta_{\chi}$ as the one obtained by minimizing CI length as described in Section 3.4).

The results below give the limiting behavior of these quantities as well as the bound on expected length in Corollary 3.3 under pointwise convergence of a sequence of functions $\omega_{n}(\delta)$ that satisfy the conditions of a modulus scaled by a sequence of constants.

Lemma G.1. Let $\omega_{n}(\delta)$ be a sequence of concave nondecreasing nonnegative functions on $[0, \infty)$ and let $\omega_{\infty}(\delta)$ be a concave nondecreasing function on $[0, \infty)$ with range $[0, \infty)$. Then the following are equivalent.

(i) For all $\delta>0, \lim _{n \rightarrow \infty} \omega_{n}(\delta)=\omega_{\infty}(\delta)$.

(ii) For all $b \in(0, \infty), b$ is in the range of $\omega_{n}$ for large enough $n$, and $\lim _{n \rightarrow \infty} \omega_{n}^{-1}(b)=$ $\omega_{\infty}^{-1}(b)$.

(iii) For any $\bar{\delta}>0, \lim _{n \rightarrow \infty} \sup _{\delta \in[0, \bar{\delta}]}\left|\omega_{n}(\delta)-\omega_{\infty}(\delta)\right|=0$.

Proof. Clearly $($ iii $) \Longrightarrow(i)$. To show $(i) \Longrightarrow($ iii $)$, given $\varepsilon>0$, let $0<\delta_{1}<\delta_{2}<\cdots<\delta_{k}=\bar{\delta}$ be such that $\omega\left(\delta_{j}\right)-\omega\left(\delta_{j-1}\right) \leq \varepsilon$ for each $j$. Then, using monotonicity of $\omega_{n}$ and $\omega_{\infty}$, we 
have $\sup _{\delta \in\left[0, \delta_{1}\right]}\left|\omega_{n}(\delta)-\omega_{\infty}(\delta)\right| \leq \max \left\{\left|\omega_{n}\left(\delta_{1}\right)\right|,\left|\omega_{n}(0)-\omega_{\infty}\left(\delta_{1}\right)\right|\right\} \rightarrow \omega_{\infty}\left(\delta_{1}\right)$ and

$$
\begin{aligned}
& \sup _{\delta \in\left[\delta_{j-1}, \delta_{j}\right]}\left|\omega_{n}(\delta)-\omega_{\infty}(\delta)\right| \leq \max \left\{\left|\omega_{n}\left(\delta_{j}\right)-\omega_{\infty}\left(\delta_{j-1}\right)\right|,\left|\omega_{n}\left(\delta_{j-1}\right)-\omega_{\infty}\left(\delta_{j}\right)\right|\right\} \\
& \rightarrow\left|\omega_{\infty}\left(\delta_{j-1}\right)-\omega_{\infty}\left(\delta_{j}\right)\right| \leq \varepsilon
\end{aligned}
$$

The result follows since $\varepsilon$ can be chosen arbitrarily small. To show $(i) \Longrightarrow(i i)$, let $\delta_{\ell}$ and $\delta_{u}$ be such that $\omega_{\infty}\left(\delta_{\ell}\right)<b<\omega_{\infty}\left(\delta_{u}\right)$. For large enough $n$, we will have $\omega_{n}\left(\delta_{\ell}\right)<b<\omega_{n}\left(\delta_{u}\right)$ so that $b$ will be in the range of $\omega_{n}$ and $\delta_{\ell}<\omega_{n}^{-1}(b)<\delta_{u}$. Since $\omega_{\infty}$ is strictly increasing, $\delta_{\ell}$ and $\delta_{u}$ can be chosen arbitrarily close to $\omega_{\infty}^{-1}(b)$, which gives the result. To show $(i i) \Longrightarrow(i)$, let $b_{\ell}$ and $b_{u}$ be such that $\omega_{\infty}^{-1}\left(b_{\ell}\right)<\delta<\omega_{\infty}^{-1}\left(b_{u}\right)$. Then, for large enough $n, \omega_{n}^{-1}\left(b_{\ell}\right)<\delta<\omega_{n}^{-1}\left(b_{u}\right)$, so that $b_{\ell}<\omega_{n}(\delta)<b_{u}$, and the result follows since $b_{\ell}$ and $b_{u}$ can be chosen arbitrarily close to $\omega_{\infty}(\delta)$ since $\omega_{\infty}^{-1}$ is strictly increasing.

Lemma G.2. Suppose that the conditions of Lemma G.1 hold with $\lim _{\delta \rightarrow 0} \omega_{\infty}(\delta)=0$ and $\lim _{\delta \rightarrow \infty} \omega_{\infty}(\delta) / \delta=0$. Let $r$ be a nonnegative function with $0 \leq r(\delta / 2) \leq \bar{r} \min \{\delta, 1\}$ for some $\bar{r}<\infty$. Then

$$
\lim _{n \rightarrow \infty} \sup _{\delta>0} \frac{\omega_{n}(\delta)}{\delta} r\left(\frac{\delta}{2}\right)=\sup _{\delta>0} \frac{\omega_{\infty}(\delta)}{\delta} r\left(\frac{\delta}{2}\right)
$$

If, in addition $r$ is continuous, $\frac{\omega_{\infty}(\delta)}{\delta} r\left(\frac{\delta}{2}\right)$ has a unique maximizer $\delta^{*}$, and, for each $n, \delta_{n}$ maximizes $\frac{\omega_{n}(\delta)}{\delta} r\left(\frac{\delta}{2}\right)$, then $\delta_{n} \rightarrow \delta^{*}$ and $\omega_{n}\left(\delta_{n}\right) \rightarrow \omega_{\infty}\left(\delta^{*}\right)$. In addition, for any $\sigma>0$ and $0<\alpha<1$ and $Z$ a standard normal variable,

$$
\lim _{n \rightarrow \infty}(1-\alpha) E\left[\omega_{n}\left(2 \sigma\left(z_{1-\alpha}-Z\right)\right) \mid Z \leq z_{1-\alpha}\right]=(1-\alpha) E\left[\omega_{\infty}\left(2 \sigma\left(z_{1-\alpha}-Z\right)\right) \mid Z \leq z_{1-\alpha}\right]
$$

Proof. We will show that the objective can be made arbitrarily small for $\delta$ outside of $[\underline{\delta}, \bar{\delta}]$ for $\underline{\delta}$ small enough and $\bar{\delta}$ large enough, and then use uniform convergence over $[\underline{\delta}, \bar{\delta}]$. First, note that, if we choose $\underline{\delta}<1$, then, for $\delta \leq \underline{\delta}$,

$$
\frac{\omega_{n}(\delta)}{\delta} r\left(\frac{\delta}{2}\right) \leq \omega_{n}(\delta) \bar{r} \leq \omega_{n}(\underline{\delta}) \bar{r} \rightarrow \omega_{\infty}(\underline{\delta}),
$$

which can be made arbitrarily small by making $\underline{\delta}$ small. Since $\omega_{n}(\delta)$ is concave and nonneg- 
ative, $\omega_{n}(\delta) / \delta$ is nonincreasing, so, for $\delta>\bar{\delta}$,

$$
\frac{\omega_{n}(\delta)}{\delta} r\left(\frac{\delta}{2}\right) \leq \frac{\omega_{n}(\delta)}{\delta} \bar{r} \leq \frac{\omega_{n}(\bar{\delta})}{\bar{\delta}} \bar{r} \rightarrow \frac{\omega_{\infty}(\bar{\delta})}{\bar{\delta}} \bar{r}
$$

which can be made arbitrarily small by making $\bar{\delta}$ large. Applying Lemma G.1 to show convergence over $[\underline{\delta}, \bar{\delta}]$ gives the first claim. The second claim follows since $\underline{\delta}$ and $\bar{\delta}$ can be chosen so that $\delta_{n} \in[\underline{\delta}, \bar{\delta}]$ for large enough $n$ (the assumption that $\frac{\omega_{\infty}(\delta)}{\delta} r\left(\frac{\delta}{2}\right)$ has a unique maximizer means that it is not identically zero), and uniform convergence to a continuous function with a unique maximizer on a compact set implies convergence of the sequence of maximizers to the maximizer of the limiting function.

For the last statement, note that, by positivity and concavity of $\omega_{n}$, we have, for large enough $n, 0 \leq \omega_{n}(\delta) \leq \omega_{n}(1) \max \{\delta, 1\} \leq\left(\omega_{\infty}(1)+1\right) \max \{\delta, 1\}$ for all $\delta>0$. The result then follows from the dominated convergence theorem.

Lemma G.3. Let $\omega_{n}(\delta)$ be a sequence of nonnegative concave functions on $[0, \infty)$ and let $\omega_{\infty}(\delta)$ be a nonnegative concave differentiable function on $[0, \infty)$. Let $\delta_{0}>0$ and suppose that $\omega_{n}(\delta) \rightarrow \omega_{\infty}(\delta)$ for all $\delta$ in a neighborhood of $\delta_{0}$. Then, for any sequence $d_{n} \in \partial \omega_{n}\left(\delta_{0}\right)$, we have $d_{n} \rightarrow \omega_{\infty}^{\prime}\left(\delta_{0}\right)$. In particular, if $\omega_{n}(\delta) \rightarrow \omega_{\infty}(\delta)$ in a neighborhood of $\delta_{0}$ and $2 \delta_{0}$, then $\frac{\omega_{n}\left(2 \delta_{0}\right)}{\omega_{n}\left(\delta_{0}\right)+\delta_{0} \omega_{n}^{\prime}\left(\delta_{0}\right)} \rightarrow \frac{\omega_{\infty}\left(2 \delta_{0}\right)}{\omega_{\infty}\left(\delta_{0}\right)+\delta_{0} \omega_{\infty}^{\prime}\left(\delta_{0}\right)}$.

Proof. By concavity, for $\eta>0$ we have $\left[\omega_{n}\left(\delta_{0}\right)-\omega_{n}\left(\delta_{0}-\eta\right)\right] / \eta \geq d_{n} \geq\left[\omega_{n}\left(\delta_{0}+\eta\right)-\omega_{n}\left(\delta_{0}\right)\right] / \eta$. For small enough $\eta$, the left and right-hand sides converge, so that $\left[\omega_{\infty}\left(\delta_{0}\right)-\omega_{\infty}\left(\delta_{0}-\eta\right)\right] / \eta \geq$ $\lim \sup _{n} d_{n} \geq \liminf _{n} d_{n} \geq\left[\omega_{\infty}\left(\delta_{0}+\eta\right)-\omega_{\infty}\left(\delta_{0}\right)\right] / \eta$. Taking the limit as $\eta \rightarrow 0$ gives the result.

\section{Appendix H Asymptotics for Regression Discontinu- ity}

This section proves Theorem E.1. We first give a general result for linear estimators under high-level conditions in Supplemental Appendix H.1. We then consider local polynomial estimators in Supplemental Appendix H.2 and optimal estimators with a plug-in variance estimate in Supplemental Appendix H.3. Theorem E.1 follows immediately from the results in these sections.

Throughout this section, we consider the RD setup where the error distribution may be non-normal as in Supplemental Appendix E.4, using the conditions in that section. We 
repeat these conditions here for convenience.

Assumption H.1. For some $p_{X,+}(0)>0$ and $p_{X,-}(0)>0$, the sequence $\left\{x_{i}\right\}_{i=1}^{n}$ satisfies $\frac{1}{n h_{n}} \sum_{i=1}^{n} m\left(x_{i} / h_{n}\right) 1\left(x_{i}>0\right) \rightarrow p_{X,+}(0) \int_{0}^{\infty} m(u) d u$ and $\frac{1}{n h_{n}} \sum_{i=1}^{n} m\left(x_{i} / h_{n}\right) 1\left(x_{i}<0\right) \rightarrow$ $p_{X,-}(0) \int_{-\infty}^{0} m(u) d u$ for any bounded function $m$ with bounded support and any $h_{n}$ with $0<\liminf _{n} h_{n} n^{1 /(2 p+1)} \leq \limsup _{n} h_{n} n^{1 /(2 p+1)}<\infty$.

Assumption H.2. For some $\sigma(x)$ with $\lim _{x \downarrow 0} \sigma(x)=\sigma_{+}(0)>0$ and $\lim _{x \uparrow 0} \sigma(x)=\sigma_{-}(0)>$ 0 , we have

(i) the $u_{i} s$ are independent under any $Q \in \mathcal{Q}_{n}$ with $E_{Q} u_{i}=0, \operatorname{var}_{Q}\left(u_{i}\right)=\sigma^{2}\left(x_{i}\right)$

(ii) for some $\eta>0, E_{Q}\left|u_{i}\right|^{2+\eta}$ is bounded uniformly over $n$ and $Q \in \mathcal{Q}_{n}$.

Theorem E.1 considers affine estimators that are optimal under the assumption that the variance function is given by $\hat{\sigma}_{+} 1(x>0)+\hat{\sigma}_{-} 1(x<0)$, which covers the plug-in optimal affine estimators used in our application. Here, it will be convenient to generalize this slightly by considering the class of affine estimators that are optimal under a variance function $\tilde{\sigma}(x)$, which may be misspecified or data-dependent, but which may take some other form. We consider two possibilities for how $\tilde{\sigma}(\cdot)$ is calibrated.

Assumption H.3. $\tilde{\sigma}(x)=\hat{\sigma}_{+} 1(x>0)+\hat{\sigma}_{-} 1(x<0)$ where $\hat{\sigma}_{+} \underset{\mathcal{F}, \mathcal{Q}_{n}}{\stackrel{p}{\rightarrow}} \tilde{\sigma}_{+}(0)>0$ and $\hat{\sigma}_{-} \underset{\mathcal{F}, \mathcal{Q}_{n}}{\stackrel{p}{\rightarrow}}$ $\tilde{\sigma}_{-}(0)>0$.

Assumption H.4. $\tilde{\sigma}(x)$ is a deterministic function with $\lim _{x \downarrow 0} \tilde{\sigma}(x)=\tilde{\sigma}_{-}(0)>0$ and $\lim _{x \uparrow 0} \tilde{\sigma}(x)=\tilde{\sigma}_{+}(0)>0$.

Assumption H.3 corresponds to the estimate of the variance function used in the application. It generalizes Assumption E.3 slightly by allowing $\hat{\sigma}_{+}$and $\hat{\sigma}_{-}$to converge to something other than the left- and right-hand limits of the true variance function. Assumption H.4 is used in deriving bounds based on infeasible estimates that use the true variance function.

Note that, under Assumption H.3, $\tilde{\sigma}_{+}(0)$ is defined as the probability limit of $\hat{\sigma}_{+}$as $n \rightarrow \infty$, and does not give the limit of $\tilde{\sigma}(x)$ as $x \downarrow 0$ (and similarly for $\tilde{\sigma}_{-}(0)$ ). We use this notation so that certain limiting quantities can be defined in the same way under each of the Assumptions H.4 and H.3. 


\section{H.1 General Results for Kernel Estimators}

We first state results for affine estimators where the weights asymptotically take a kernel form. We consider a sequence of estimators of the form

$$
\hat{L}=\frac{\sum_{i=1}^{n} k_{n}^{+}\left(x_{i} / h_{n}\right) 1\left(x_{i}>0\right) y_{i}}{\sum_{i=1}^{n} k_{n}^{+}\left(x_{i} / h_{n}\right) 1\left(x_{i}>0\right)}-\frac{\sum_{i=1}^{n} k_{n}^{-}\left(x_{i} / h_{n}\right) 1\left(x_{i}<0\right) y_{i}}{\sum_{i=1}^{n} k_{n}^{-}\left(x_{i} / h_{n}\right) 1\left(x_{i}<0\right)}
$$

where $k_{n}^{+}$and $k_{n}^{-}$are sequences of kernels. The assumption that the same bandwidth is used on each side of the discontinuity is a normalization: it can always be satisfied by redefining one of the kernels $k_{n}^{+}$or $k_{n}^{-}$. We make the following assumption on the sequence of kernels.

Assumption H.5. The sequences of kernels and bandwidths $k_{n}^{+}$and $h_{n}$ satisfy

(i) $k_{n}^{+}$has support bounded uniformly over $n$. For a bounded kernel $k^{+}$with $\int k^{+}(u) d u>$ 0 , we have $\sup _{x}\left|k_{n}^{+}(x)-k^{+}(x)\right| \rightarrow 0$

(ii) $\frac{1}{n h_{n}} \sum_{i=1}^{n} k_{n}^{+}\left(x_{i} / h_{n}\right) 1\left(x_{i}>0\right)\left(x_{i}, \ldots, x_{i}^{p-1}\right)^{\prime}=0$ for each $n$

(iii) $h_{n} n^{1 /(2 p+1)} \rightarrow h_{\infty}$ for some constant $0<h_{\infty}<\infty$,

and similarly for $k_{n}^{-}$for some $k^{-}$.

Let

$$
\begin{aligned}
& \overline{\operatorname{bias}}_{n}=\frac{\sum_{i=1}^{n}\left|k_{n}^{+}\left(x_{i} / h_{n}\right)\right| 1\left(x_{i}>0\right) C\left|x_{i}\right|^{p}}{\sum_{i=1}^{n} k_{n}^{+}\left(x_{i} / h_{n}\right) 1\left(x_{i}>0\right)}+\frac{\sum_{i=1}^{n}\left|k_{n}^{-}\left(x_{i} / h_{n}\right)\right| 1\left(x_{i}<0\right) C\left|x_{i}\right|^{p}}{\sum_{i=1}^{n} k_{n}^{-}\left(x_{i} / h_{n}\right) 1\left(x_{i}<0\right)} \\
& =C h_{n}^{p}\left(\frac{\sum_{i=1}^{n}\left|k_{n}^{+}\left(x_{i} / h_{n}\right)\right| 1\left(x_{i}>0\right)\left|x_{i} / h_{n}\right|^{p}}{\sum_{i=1}^{n} k_{n}^{+}\left(x_{i} / h_{n}\right) 1\left(x_{i}>0\right)}+\frac{\sum_{i=1}^{n}\left|k_{n}^{-}\left(x_{i} / h_{n}\right)\right| 1\left(x_{i}<0\right)\left|x_{i} / h_{n}\right|^{p}}{\sum_{i=1}^{n} k_{n}^{-}\left(x_{i} / h_{n}\right) 1\left(x_{i}<0\right)}\right)
\end{aligned}
$$

and

$$
\begin{aligned}
v_{n} & =\frac{\sum_{i=1}^{n} k_{n}^{+}\left(x_{i} / h_{n}\right)^{2} 1\left(x_{i}>0\right) \sigma^{2}\left(x_{i}\right)}{\left[\sum_{i=1}^{n} k_{n}^{+}\left(x_{i} / h_{n}\right) 1\left(x_{i}>0\right)\right]^{2}}+\frac{\sum_{i=1}^{n} k_{n}^{-}\left(x_{i} / h_{n}\right)^{2} 1\left(x_{i}<0\right) \sigma^{2}\left(x_{i}\right)}{\left[\sum_{i=1}^{n} k_{n}^{-}\left(x_{i} / h_{n}\right) 1\left(x_{i}<0\right)\right]^{2}} \\
& =\frac{1}{n h_{n}}\left(\frac{\frac{1}{n h_{n}} \sum_{i=1}^{n} k_{n}^{+}\left(x_{i} / h_{n}\right)^{2} 1\left(x_{i}>0\right) \sigma^{2}\left(x_{i}\right)}{\left[\frac{1}{n h_{n}} \sum_{i=1}^{n} k_{n}^{+}\left(x_{i} / h_{n}\right) 1\left(x_{i}>0\right)\right]^{2}}+\frac{\frac{1}{n h_{n}} \sum_{i=1}^{n} k_{n}^{-}\left(x_{i} / h_{n}\right)^{2} 1\left(x_{i}<0\right) \sigma^{2}\left(x_{i}\right)}{\left[\frac{1}{n h_{n}} \sum_{i=1}^{n} k_{n}^{-}\left(x_{i} / h_{n}\right) 1\left(x_{i}<0\right)\right]^{2}}\right)
\end{aligned}
$$

Note that $v_{n}$ is the (constant over $Q \in \mathcal{Q}_{n}$ ) variance of $\hat{L}$, and that, by arguments in Supplemental Appendix E.1, $\overline{\operatorname{bias}}_{n}=\sup _{f \in \mathcal{F}}\left(E_{f, Q} \hat{L}-L f\right)=-\inf _{f \in \mathcal{F}}\left(E_{f, Q} \hat{L}-L f\right)$ for any $Q \in \mathcal{Q}_{n}$ under Assumption H.5 (ii). 
To form a feasible CI, we need an estimate of $v_{n}$. While the results below go through with any variance estimate that is consistent uniformly over $f, \mathcal{Q}_{n}$, we propose one here for concreteness. For a possibly data-dependent guess $\tilde{\sigma}(\cdot)$ of the variance function, let $\tilde{v}_{n}$ denote $v_{n}$ with $\sigma(\cdot)$ replaced by $\tilde{\sigma}(\cdot)$. We record the limiting behavior of $\overline{\operatorname{bias}}_{n}, v_{n}$ and $\tilde{v}_{n}$ in the following lemma. Let

$$
\overline{\operatorname{bias}}_{\infty}=C h_{\infty}^{p}\left(\frac{\int_{0}^{\infty}\left|k^{+}(u)\right||u|^{p} d u}{\int_{0}^{\infty} k^{+}(u) d u}+\frac{\int_{-\infty}^{0}\left|k^{-}(u)\right||u|^{p} d u}{\int_{-\infty}^{0} k^{-}(u) d u}\right)
$$

and

$$
v_{\infty}=\frac{1}{h_{\infty}}\left(\frac{\sigma_{+}^{2}(0) \int_{0}^{\infty} k^{+}(u)^{2} d u}{p_{X,+}(0)\left[\int_{0}^{\infty} k^{+}(u) d u\right]^{2}}+\frac{\sigma_{-}^{2}(0) \int_{-\infty}^{0} k^{-}(u)^{2} d u}{p_{X,-}(0)\left[\int_{-\infty}^{0} k^{-}(u) d u\right]^{2}}\right) .
$$

Lemma H.1. Suppose that Assumption H.1 holds. If Assumption H.5 also holds, then $\lim _{n \rightarrow \infty} n^{p /(2 p+1)} \overline{b i a s}_{n}=\overline{b i a s}_{\infty}$ and $\lim _{n \rightarrow \infty} n^{2 p /(2 p+1)} v_{n}=v_{\infty}$. If, in addition, $\tilde{\sigma}(\cdot)$ satisfies Assumption H.3 or Assumption H.4 with $\tilde{\sigma}_{+}(0)=\sigma_{+}(0)$ and $\tilde{\sigma}_{-}(0)=\sigma_{-}(0)$, then $n^{2 p /(2 p+1)} \tilde{v}_{n} \underset{\mathcal{F}, \mathcal{Q}_{n}}{\stackrel{p}{\rightarrow}} v_{\infty}$ under Assumption H.3 and $\lim _{n \rightarrow \infty} n^{2 p /(2 p+1)} \tilde{v}_{n}=v_{\infty}$ under Assumption H.4.

Proof. The results follow from applying the convergence in Assumption H.1 along with Assumption H.5(i) to the relevant terms in $\overline{\operatorname{bias}}_{n}$ and $\tilde{v}_{n}$.

Theorem H.1. Suppose that Assumptions H.1, H.2 and H.5 hold, and that $\tilde{v}_{n}$ is formed using a variance function $\tilde{\sigma}(\cdot)$ that satisfies Assumption H.3 or H.4 with $\tilde{\sigma}_{+}(0)=\sigma_{+}(0)$ and $\tilde{\sigma}_{-}(0)=\sigma_{-}(0)$. Then

$$
\liminf _{n \rightarrow \infty} \inf _{f \in \mathcal{F}_{R D T, p}(C), Q \in \mathcal{Q}_{n}} P_{f, Q}\left(L f \in\left\{\hat{L} \pm \mathrm{cv}_{\alpha}\left(\overline{\text { bias }}_{n} / \tilde{v}_{n}\right) \sqrt{\tilde{v}_{n}}\right\}\right) \geq 1-\alpha
$$

and, letting $\hat{c}=\hat{L}-\overline{b i a s}_{n}-z_{1-\alpha} \sqrt{\tilde{v}_{n}}$,

$$
\liminf _{n \rightarrow \infty} \inf _{f \in \mathcal{F}_{R D T, p}(C), Q \in \mathcal{Q}_{n}} P_{f, Q}(L f \in[\hat{c}, \infty)) \geq 1-\alpha
$$

In addition, $n^{p /(2 p+1)} \mathrm{cv}_{\alpha}\left(\overline{\text { bias }}_{n} / \tilde{v}_{n}\right) \tilde{v}_{n} \underset{\mathcal{F}, \mathcal{Q}_{n}}{\stackrel{p}{\rightarrow}} \mathrm{cv}_{\alpha}\left(\overline{\text { bias }}_{\infty} / v_{\infty}\right) v_{\infty}$ if $\tilde{\sigma}(\cdot)$ satisfies Assumption H.3 and $n^{p /(2 p+1)} \mathrm{cv}_{\alpha}\left(\overline{\text { bias }}_{n} / \tilde{v}_{n}\right) \tilde{v}_{n} \rightarrow \mathrm{cv}_{\alpha}\left(\overline{\text { bias }}_{\infty} / v_{\infty}\right) v_{\infty}$ if $\tilde{\sigma}(\cdot)$ satisfies Assumption H.4. The 
minimax $\beta$ quantile of the one-sided CI satisfies

$$
\limsup _{n \rightarrow \infty} n^{p /(2 p+1)} \sup _{f \in \mathcal{F}_{R D T, p}(C), Q \in \mathcal{Q}_{n}} q_{f, Q, \beta}(L f-\hat{c}) \leq 2 \overline{b i a s}_{\infty}+\left(z_{\beta}+z_{1-\alpha}\right) \sqrt{v_{\infty}} .
$$

The worst-case $\beta$ quantile over $\mathcal{F}_{R D T, p}(0)$ satisfies

$$
\limsup _{n \rightarrow \infty} n^{p /(2 p+1)} \sup _{f \in \mathcal{F}_{R D T, p}(0), Q \in \mathcal{Q}_{n}} q_{f, Q, \beta}(L f-\hat{c}) \leq \overline{\operatorname{bias}}{ }_{\infty}+\left(z_{\beta}+z_{1-\alpha}\right) \sqrt{v_{\infty}}
$$

Furthermore, the same holds with $\hat{L}, \overline{\text { bias }}_{n}$ and $\tilde{v}_{n}$ replaced by any $\hat{L}^{*}, \overline{\text { bias }}_{n}^{*}$ and $\tilde{v}_{n}^{*}$ such that

$$
n^{p /(2 p+1)}\left(\hat{L}-\hat{L}^{*}\right) \underset{\mathcal{F}, \mathcal{Q}_{n}}{\stackrel{p}{\rightarrow}} 0, \quad n^{p /(2 p+1)}\left(\overrightarrow{\text { bias }}_{n}-{\overrightarrow{b i a s_{n}}}_{n}^{*} \underset{\mathcal{F}, \mathcal{Q}_{n}}{\stackrel{p}{\rightarrow}} 0, \quad \frac{\tilde{v}_{n}}{\tilde{v}_{n}^{*}} \underset{\mathcal{F}, \mathcal{Q}_{n}}{\stackrel{p}{\rightarrow}} 1 .\right.
$$

Proof. We verify the conditions of Theorem F.1. Condition (S14) follows from Lemma H.1. To verify (S13), note that $\hat{L}$ takes the general form in Theorem F.1 with $w_{n, i}$ given by $w_{n, i}=$ $k_{n}^{+}\left(x_{i} / h_{n}\right) / \sum_{j=1}^{n} k_{n}^{+}\left(x_{j} / h_{n}\right) 1\left(x_{j}>0\right)$ for $x_{i}>0$ and $w_{n, i}=k_{n}^{-}\left(x_{i} / h_{n}\right) / \sum_{j=1}^{n} k_{n}^{-}\left(x_{j} / h_{n}\right)$. $1\left(x_{j}<0\right)$ for $x_{i}<0$. The uniform central limit theorem in (S13) with $w_{n, i}$ taking this form follows from Lemma F.1. This gives the asymptotic coverage statements.

For the asymptotic formulas for excess length of the one-sided CI and length of the two-sided CI, we apply Theorem F.2 with $n^{-p /(2 p+1)} \overline{\operatorname{bias}}_{\infty}$ playing the role of ${\widetilde{\operatorname{bias}_{n}}}_{n}$ and $n^{-p /(2 p+1)} v_{\infty}$ playing the role of $\widetilde{\mathrm{se}}_{n}$. Finally, the last statement of the theorem is immediate from Theorem F.2.

\section{H.2 Local Polynomial Estimators}

The $(p-1)$ th order local polynomial estimator of $f_{+}(0)$ based on kernel $k_{+}^{*}$ and bandwidth $h_{+, n}$ is given by

$$
\begin{aligned}
\hat{f}_{+}(0)= & e_{1}^{\prime}\left(\sum_{i=1}^{n} p\left(x_{i} / h_{+, n}\right) p\left(x_{i} / h_{+, n}\right)^{\prime} k_{+}^{*}\left(x_{i} / h_{+, n}\right) 1\left(x_{i}>0\right)\right)^{-1} \\
& \sum_{i=1}^{n} k_{+}^{*}\left(x_{i} / h_{+, n}\right) 1\left(x_{i}>0\right) p\left(x_{i} / h_{+, n}\right) y_{i}
\end{aligned}
$$

where $e_{1}=(1,0, \ldots, 0)^{\prime}$ and $p(x)=\left(1, x, x^{2}, \ldots, x^{p-1}\right)^{\prime}$. Letting the local polynomial estimator of $f_{-}(0)$ be defined analogously for some kernel $k_{-}^{*}$ and bandwidth $h_{-, n}$, the local 
polynomial estimator of $L f=f_{+}(0)-f_{-}(0)$ is given by

$$
\hat{L}=\hat{f}_{+}(0)-\hat{f}_{-}(0)
$$

This takes the form given in Supplemental Appendix H.1, with $h_{n}=h_{n,+}$,

$$
k_{n}^{+}(u)=e_{1}^{\prime}\left(\frac{1}{n h_{n}} \sum_{i=1}^{n} p\left(x_{i} / h_{+, n}\right) p\left(x_{i} / h_{+, n}\right)^{\prime} k_{+}^{*}\left(x_{i} / h_{+, n}\right) 1\left(x_{i}>0\right)\right)^{-1} k_{+}^{*}(u) p(u) 1(u>0)
$$

and

$$
\begin{aligned}
k_{n}^{-}(u)= & e_{1}^{\prime}\left(\frac{1}{n h_{n}} \sum_{i=1}^{n} p\left(x_{i} / h_{-, n}\right) p\left(x_{i} / h_{-, n}\right)^{\prime} k_{+}^{*}\left(x_{i} / h_{-, n}\right) 1\left(x_{i}<0\right)\right)^{-1} \\
& k_{+}^{*}\left(u\left(h_{n,+} / h_{n,-}\right)\right) p\left(u\left(h_{n,+} / h_{n,-}\right)\right) 1(u<0) .
\end{aligned}
$$

Let $M^{+}$be the $(p-1) \times(p-1)$ matrix with $\int_{0}^{\infty} u^{j+k-2} k_{+}^{*}(u)$ as the $i, j$ th entry, and let $M^{-}$be the $(p-1) \times(p-1)$ matrix with $\int_{-\infty}^{0} u^{j+k-2} k_{-}^{*}(u)$ as the $i, j$ th entry. Under Assumption H.1, for $k_{+}^{*}$ and $k_{-}^{*}$ bounded with bounded support, $\frac{1}{n h_{n}} \sum_{i=1}^{n} p\left(x_{i} / h_{+, n}\right) p\left(x_{i} / h_{+, n}\right)^{\prime} k_{+}^{*}\left(x_{i} / h_{+, n}\right)$. $1\left(x_{i}>0\right) \rightarrow M^{+} p_{X,+}(0)$ and similarly $\frac{1}{n h_{n}} \sum_{i=1}^{n} p\left(x_{i} / h_{-, n}\right) p\left(x_{i} / h_{-, n}\right)^{\prime} k_{+}^{*}\left(x_{i} / h_{-, n}\right) \cdot 1\left(x_{i}<\right.$ $0) \rightarrow M^{-} p_{X,-}(0)$. Furthermore, Assumption H.5 (ii) follows immediately from the normal equations for the local polynomial estimator. This gives the following result.

Theorem H.2. Let $k_{+}^{*}$ and $k_{-}^{*}$ be bounded and uniformly continuous with bounded support. Let $h_{n,+} n^{1 /(2 p+1)} \rightarrow h_{\infty}>0$ and suppose $h_{n,-} / h_{n,+}$ converges to a strictly positive constant. Then Assumption H.5 holds for the local polynomial estimator so long as Assumption H.1 holds.

\section{H.3 Optimal Affine Estimators}

We now consider the class of affine estimators that are optimal under the assumption that the variance function is given by $\tilde{\sigma}(\cdot)$, which satisfies either Assumption H.3 or Assumption H.4. We use the same notation as in Supplemental Appendix E, except that $n$ and/or $\tilde{\sigma}(\cdot)$ are added as subscripts for many of the objects under consideration to make the dependence on $\left\{x_{i}\right\}_{i=1}^{n}$ and $\tilde{\sigma}(\cdot)$ explicit.

The modulus problem is given by Equation (S3) in Supplemental Appendix E.2 with $\tilde{\sigma}(\cdot)$ in place of $\sigma(\cdot)$. We use $\omega_{\tilde{\sigma}(\cdot), n}(\delta)$ to denote the modulus, or $\omega_{n}(\delta)$ when the context is 
clear. The corresponding estimator $\hat{L}_{\delta, \tilde{\sigma}(\cdot)}$ is then given by Equation (S10) in Supplemental Appendix E.2 with $\tilde{\sigma}(\cdot)$ in place of $\sigma(\cdot)$.

We will deal with the inverse modulus, and use Lemma G.1 to obtain results for the modulus itself. The inverse modulus $\omega_{\tilde{\sigma}(\cdot), n}^{-1}(2 b)$ is given by Equation (S9) in Supplemental Appendix E.2, with $\tilde{\sigma}^{2}\left(x_{i}\right)$ in place of $\sigma^{2}\left(x_{i}\right)$, and the solution takes the form given in that section. Let $h_{n}=n^{-1 /(2 p+1)}$. We will consider a sequence $b=b_{n}$, and will define $\tilde{b}_{n}=n^{p /(2 p+1)} b_{n}=h_{n}^{-p} b_{n}$. Under Assumption H.4, we will assume that $\tilde{b}_{n} \rightarrow \tilde{b}_{\infty}$ for some $\tilde{b}_{\infty}>0$. Under Assumption H.3, we will assume that $\tilde{b}_{n} \underset{\mathcal{F}, \mathcal{Q}_{n}}{\stackrel{p}{\rightarrow}} \tilde{b}_{\infty}$ for some $\tilde{b}_{\infty}>0$. We will then show that this indeed holds for $2 b_{n}=\omega_{\tilde{\sigma}(\cdot), n}\left(\delta_{n}\right)$ with $\delta_{n}$ chosen as in Theorem H.3 below.

Let $\tilde{b}_{n}=n^{p /(2 p+1)} b_{n}=h_{n}^{-p} b_{n}, \tilde{b}_{-, n}=n^{p /(2 p+1)} b_{-, n}=h_{n}^{-p} b_{-, n}, \tilde{d}_{+, j, n}=n^{(p-j) /(2 p+1)} d_{+, j, n}=$ $h_{n}^{j-p} d_{+, j, n}$ and $\tilde{d}_{-, j, n}=n^{(p-j) /(2 p+1)} d_{-, j, n}=h_{n}^{j-p} d_{-, j, n}$ for $j=1, \ldots, p-1$, where $b_{n}, b_{-, n}$, $d_{+, n}$, and $d_{-n}$ correspond to the function $g_{b, C}$ that solves the inverse modulus problem, given in Supplemental Appendix E.2. These values of $\tilde{b}_{+, n}, \tilde{b}_{-, n}, \tilde{d}_{+, n}$ and $\tilde{d}_{-, n}$ minimize $G_{n}\left(b_{+}, b_{-}, d_{+}, d_{-}\right)$subject to $b_{+}+b_{-}=\tilde{b}_{n}$ where, letting $\mathcal{A}\left(x_{i}, b, d\right)=h_{n}^{p} b+\sum_{j=1}^{p-1} h_{n}^{p-j} d_{j} x_{i}^{j}$,

$$
\begin{aligned}
& G_{n}\left(b_{+}, b_{-}, d_{+}, d_{-}\right)= \\
& \quad \sum_{i=1}^{n} \tilde{\sigma}^{-2}\left(x_{i}\right)\left(\left(\mathcal{A}\left(x_{i}, b_{+}, d_{+}\right)-C\left|x_{i}^{p}\right|\right)_{+}+\left(\mathcal{A}\left(x_{i}, b_{+}, d_{+}\right)+C\left|x_{i}\right|^{p}\right)_{-}\right)^{2} 1\left(x_{i}>0\right) \\
& +\sum_{i=1}^{n} \tilde{\sigma}^{-2}\left(x_{i}\right)\left(\left(\mathcal{A}\left(x_{i}, b_{-}, d_{-}\right)-C\left|x_{i}\right|^{p}\right)+\left(\mathcal{A}\left(x_{i}, b_{-}, d_{-}\right)+C\left|x_{i}\right|^{p}\right)_{-}\right)^{2} 1\left(x_{i}<0\right) \\
& \quad=\frac{1}{n h_{n}} \sum_{i=1}^{n} k_{\tilde{\sigma}(\cdot)}^{+}\left(x_{i} / h_{n} ; b_{+}, d_{+}\right)^{2} \tilde{\sigma}^{2}\left(x_{i}\right)+\frac{1}{n h_{n}} \sum_{i=1}^{n} k_{\tilde{\sigma}(\cdot)}^{-}\left(x_{i} / h_{n} ; b_{-}, d_{-}\right)^{2} \tilde{\sigma}^{2}\left(x_{i}\right)
\end{aligned}
$$

with

$$
\begin{aligned}
& k_{\tilde{\sigma}(\cdot)}^{+}(u ; b, d)=\tilde{\sigma}^{-2}\left(u h_{n}\right)\left(\left(b+\sum_{j=1}^{p-1} d_{j} u^{j}-C|u|^{p}\right)_{+}-\left(b+\sum_{j=1}^{p-1} d_{j} u^{j}+C|u|^{p}\right)\right) 1(u>0), \\
& k_{\tilde{\sigma}(\cdot)}^{-}(u ; b, d)=\tilde{\sigma}^{-2}\left(u h_{n}\right)\left(\left(b+\sum_{j=1}^{p-1} d_{j} u^{j}-C|u|^{p}\right)_{+}-\left(b+\sum_{j=1}^{p-1} d_{j} u^{j}+C|u|^{p}\right)_{-}\right) 1(u<0) .
\end{aligned}
$$

We use the notation $k_{c}^{+}$for a scalar $c$ to denote $k_{\tilde{\sigma}(\cdot)}^{+}$where $\tilde{\sigma}(\cdot)$ is given by the constant function $\tilde{\sigma}(x)=c$.

With these definitions, the estimator $\hat{L}_{\delta, \tilde{\sigma}(\cdot)}$ with $\omega_{\tilde{\sigma}(\cdot), n}(\delta)=2 b_{n}$ takes the general kernel 
form in Supplemental Appendix H.1 with $k_{n}^{+}(u)=k_{\tilde{\sigma}(\cdot)}^{+}\left(u ; \tilde{b}_{+, n}, \tilde{d}_{+, n}\right)$ and similarly for $k_{n}^{-}$. In the notation of Supplemental Appendix H.1, $\overline{\operatorname{bias}}_{n}$ is given by $\frac{1}{2}\left(\omega_{\tilde{\sigma}(\cdot), n}(\delta)-\delta \omega_{\tilde{\sigma}(\cdot), n}^{\prime}(\delta)\right)$ and $\tilde{v}_{n}$ is given by $\omega_{\tilde{\sigma}(\cdot), n}^{\prime}(\delta)^{2}$ (see Equation (24) in the main text). If $\delta$ is chosen to minimize the length of the fixed-length CI, the half-length will be given by

$$
\mathrm{cv}_{\alpha}\left(\overline{\operatorname{bias}}_{n} / \sqrt{\tilde{v}_{n}}\right) \sqrt{\tilde{v}_{n}}=\inf _{\delta>0} \operatorname{cv}_{\alpha}\left(\frac{\omega_{\tilde{\sigma}(\cdot), n}(\delta)}{2 \omega_{\tilde{\sigma}(\cdot), n}^{\prime}(\delta)}-\frac{\delta}{2}\right) \omega_{\tilde{\sigma}(\cdot), n}^{\prime}(\delta),
$$

and $\delta$ will achieve the minimum in the above display. Similarly, if the MSE criterion is used, $\delta$ will minimize $\overline{\operatorname{bias}}_{n}^{2}+v_{n}$.

We proceed by verifying the conditions of Theorem H.1 for the case where $\tilde{\sigma}(\cdot)$ is nonrandom and satisfies Assumption H.4, and then verifying the conditions in the last display of Theorem H.1 for the case where $\tilde{\sigma}(\cdot)$ satisfies Assumption H.3. The limiting kernel $k^{+}$and $k^{-}$in Assumption H.5 will correspond to an asymptotic version of the modulus problem, which we now describe. Let

$$
\begin{aligned}
G_{\infty}\left(b_{+}, b_{-}, d_{+}, d_{-}\right)=p_{X,+}(0) \int_{0}^{\infty} \tilde{\sigma}_{+}^{2}(0) k_{\tilde{\sigma}_{+}(0)}^{+}\left(u ; b_{+}, d_{+}\right)^{2} d u & \\
& +p_{X,-}(0) \int_{0}^{\infty} \tilde{\sigma}_{-}^{2}(0) k_{\tilde{\sigma}_{-}(0)}^{+}\left(u ; b_{+}, d_{+}\right)^{2} d u .
\end{aligned}
$$

Consider the limiting inverse modulus problem

$$
\begin{aligned}
\omega_{\tilde{\sigma}_{+}(0), \tilde{\sigma}_{-}(0), \infty}\left(2 \tilde{b}_{\infty}\right)= & \min _{f_{+}, f_{-} \in \mathcal{F}_{R D T, p}(C)} \sqrt{\frac{p_{X,+}(0)}{\tilde{\sigma}_{+}^{2}(0)} \int_{0}^{\infty} f_{+}(u)^{2} d u+\frac{p_{X,-}(0)}{\tilde{\sigma}_{-}^{2}(0)} \int_{-\infty}^{0} f_{-}(u)^{2} d u} \\
& \text { s.t. } f_{+}(0)+f_{-}(0) \geq \tilde{b}_{\infty} .
\end{aligned}
$$

We use $\omega_{\infty}(\delta)=\omega_{\tilde{\sigma}_{+}(0), \tilde{\sigma}_{-}(0), \infty}(\delta)$ to denote the limiting modulus corresponding to this inverse modulus. The limiting inverse modulus problem is solved by the functions $f_{+}(u)=$ $\tilde{\sigma}_{+}^{2}(0) k_{\tilde{\sigma}_{+}(0)}^{+}\left(u ; b_{+}, d_{+}\right)=k_{1}^{+}\left(u ; b_{+}, d_{+}\right)$and $f_{-}(u)=\tilde{\sigma}_{-}^{2}(0) k_{\tilde{\sigma}_{-}(0)}^{+}\left(u ; b_{-}, d_{-}\right)=k_{1}^{-}\left(u ; b_{+}, d_{+}\right)$ for some $\left(b_{+}, b_{-}, d_{+}, d_{-}\right)$with $b_{+}+b_{-}=\tilde{b}_{\infty}$ (this holds by the same arguments as for the modulus problem in Supplemental Appendix E.2). Thus, for any minimizer of $G_{\infty}$, the functions $k_{1}^{+}\left(\cdot ; b_{+}, d_{+}\right)$and $k_{1}^{+}\left(\cdot ; b_{+}, d_{+}\right)$must solve the above inverse modulus problem. The solution to this problem is unique by strict convexity, which implies that $G_{\infty}$ has a unique minimizer. Similarly, the minimizer of $G_{n}$ is unique for each $n$. Let $\left(\tilde{b}_{+, \infty}, \tilde{b}_{-, \infty}, \tilde{d}_{+, \infty}, \tilde{d}_{-, \infty}\right)$ denote the minimizer of $G_{\infty}$. The limiting kernel $k^{+}$in Assumption H.5 will be given by 
$k_{\tilde{\sigma}_{+}(0)}^{+}\left(\cdot ; \tilde{b}_{+, \infty}, \tilde{d}_{+, \infty}\right)$ and similarly for $k^{-}$.

To derive the form of the limiting modulus of continuity, we argue as in Donoho and Low (1992). Let $k_{1}^{+}\left(\cdot ; \tilde{b}_{+, \infty, 1}, \tilde{d}_{+, \infty, 1}\right)$ and $k_{1}^{+}\left(\cdot ; \tilde{b}_{+, \infty, 1}, \tilde{d}_{+, \infty, 1}\right)$ solve the inverse modulus problem $\omega_{\infty}^{-1}\left(2 \tilde{b}_{\infty}\right)$ for $\tilde{b}_{\infty}=1$. The feasible set for a given $\tilde{b}_{\infty}$ consists of all $b_{+}, b_{-}, d_{+}, d_{-}$such that $b_{+}+b_{-} \geq \tilde{b}_{\infty}$, and a given $b_{+}, b_{-}, d_{+}, d_{-}$in this set achieves the value

$$
\begin{aligned}
& \sqrt{\frac{p_{X,+}(0)}{\tilde{\sigma}_{+}^{2}(0)} \int_{0}^{\infty} k_{1}^{+}\left(u ; b_{+}, d_{+}\right)^{2} d u+\frac{p_{X,-}(0)}{\tilde{\sigma}_{-}^{2}(0)} \int_{-\infty}^{0} k_{1}^{-}\left(u ; b_{-}, d_{-}\right)^{2} d u} \\
& =\sqrt{\frac{p_{X,+}(0)}{\tilde{\sigma}_{+}^{2}(0)} \int_{0}^{\infty} k_{1}^{+}\left(v b_{\infty}^{1 / p} ; b_{+}, d_{+}\right)^{2} d\left(v b_{\infty}^{1 / p}\right)+\frac{p_{X,-}(0)}{\tilde{\sigma}_{-}^{2}(0)} \int_{-\infty}^{0} k_{1}^{-}\left(v b_{\infty}^{1 / p} ; b_{-}, d_{-}\right)^{2} d\left(v b_{\infty}^{1 / p}\right)} \\
& =\sqrt{\frac{p_{X,+}(0)}{\tilde{\sigma}_{+}^{2}(0)} \tilde{b}_{\infty}^{1 / p} \int_{0}^{\infty} \tilde{b}_{\infty}^{2} k_{1}^{+}\left(v ; b_{+} / \tilde{b}_{\infty}, \bar{d}_{+}\right)^{2} d v+\frac{p_{X,-}(0)}{\tilde{\sigma}_{-}^{2}(0)} \tilde{b}_{\infty}^{1 / p} \int_{-\infty}^{0} \tilde{b}_{\infty}^{2} k_{1}^{-}\left(v ; b_{-} / \tilde{b}_{\infty}, \bar{d}_{-}\right)^{2} d v}
\end{aligned}
$$

where $\bar{d}_{+}=\left(d_{+, 1} / \tilde{b}_{\infty}^{(p-1) / p}, \ldots, d_{+, p-1} / \tilde{b}_{\infty}^{1 / p}\right)^{\prime}$ and similarly for $\bar{d}_{-}$. This uses the fact that, for any $h>0, h^{p} k_{1}^{+}\left(u / h ; b_{+}, d_{+}\right)=k_{1}^{+}\left(u ; b_{+} h^{p}, d_{+, 1} h^{p-1}, d_{+, 2} h^{p-2}, \ldots, d_{+, p-1} h\right)$ and similarly for $k_{1}^{-}$. This can be seen to be $\tilde{b}_{\infty}^{(2 p+1) /(2 p)}$ times the objective evaluated at $\left(b_{+} / \tilde{b}_{\infty}, b_{-} / \tilde{b}_{\infty}, \bar{d}_{+}, \bar{d}_{-}\right)$, which is feasible under $\tilde{b}_{\infty}=1$. Similarly, for any feasible function under $\tilde{b}_{\infty}=1$, there is a feasible function under a given $\tilde{b}_{\infty}$ that achieves $\tilde{b}_{\infty}^{(2 p+1) /(2 p)}$ times the value of under $\tilde{b}_{\infty}=1$. It follows that $\omega_{\infty}^{-1}(2 b)=b^{(2 p+1) /(2 p)} \omega_{\infty}(2)$. Thus, $\omega_{\infty}^{-1}$ is invertible and the inverse $\omega_{\infty}$ satisfies $\omega_{\infty}(\delta)=\omega_{\tilde{\sigma}_{+}(0), \tilde{\sigma}_{-}(0), \infty}(\delta)=\delta^{2 p /(2 p+1)} \omega_{\tilde{\sigma}_{+}(0), \tilde{\sigma}_{-}(0), \infty}(1)$.

If $\tilde{b}_{\infty}=\omega_{\infty}\left(\delta_{\infty}\right)$ for some $\delta_{\infty}$, then it can be checked that the limiting variance and worst-case bias defined in Supplemental Appendix H.1 correspond to the limiting modulus problem:

$$
\overline{\operatorname{bias}}_{\infty}=\frac{1}{2}\left(\omega_{\infty}\left(\delta_{\infty}\right)-\delta_{\infty} \omega_{\infty}^{\prime}\left(\delta_{\infty}\right)\right), \quad \sqrt{v_{\infty}}=\omega_{\infty}^{\prime}\left(\delta_{\infty}\right)
$$

Furthermore, we will show that, if $\delta$ is chosen to optimize FLCI length for $\omega_{\tilde{\sigma}(\cdot), n}$, then this will hold with $\delta_{\infty}$ optimizing $\mathrm{cv}_{\alpha}\left(\overline{\operatorname{bias}}_{\infty} / \sqrt{v_{\infty}}\right) \sqrt{v_{\infty}}$. Similarly, if $\delta$ is chosen to optimize MSE for $\omega_{\tilde{\sigma}(\cdot), n}$, then this will hold with $\delta_{\infty}$ optimizing $\overline{\operatorname{bias}}_{\infty}^{2}+v_{\infty}$.

We are now ready to state the main result concerning the asymptotic validity and efficiency of feasible CIs based on the estimator given in this section.

Theorem H.3. Suppose Assumptions H.1 and H.2 hold. Let $\hat{L}=\hat{L}_{\delta_{n}, \tilde{\sigma}(\cdot)}$ where $\delta_{n}$ is chosen to optimize one of the performance criteria for $\omega_{\tilde{\sigma}(\cdot), n}$ (FLCI length, RMSE, or a given quantile of excess length), and suppose that $\tilde{\sigma}(\cdot)$ satisfies Assumption H.3 or Assumption H.4 
with $\tilde{\sigma}_{+}(0)=\sigma_{+}(0)$ and $\tilde{\sigma}_{+}(0)=\sigma_{-}(0)$. Let $\overline{\operatorname{bias}}_{n}=\frac{1}{2}\left(\omega_{\tilde{\sigma}(\cdot), n}\left(\delta_{n}\right)-\delta_{n} \omega_{\tilde{\sigma}(\cdot), n}^{\prime}\left(\delta_{n}\right)\right)$ and $\tilde{v}_{n}=$ $\omega_{\tilde{\sigma}(\cdot), n}^{\prime}\left(\delta_{n}\right)^{2}$ denote the worst-case bias and variance formulas. Let $\hat{c}_{\alpha, \delta_{n}}=\hat{L}-\overline{b i a s}_{n}-z_{1-\alpha} \sqrt{\tilde{v}_{n}}$ and $\hat{\chi}=\mathrm{cv}_{\alpha}\left(\overline{\text { bias }}_{n} / \sqrt{\tilde{v}_{n}}\right) \sqrt{\tilde{v}_{n}}$ so that $\left[\hat{c}_{\alpha, \delta_{n}}, \infty\right)$ and $[\hat{L}-\hat{\chi}, \hat{L}+\hat{\chi}]$ give the corresponding CIs.

The $C I s\left[\hat{c}_{\alpha, \delta_{n}}, \infty\right)$ and $[\hat{L}-\hat{\chi}, \hat{L}+\hat{\chi}]$ have uniform asymptotic coverage at least $1-\alpha$. In addition, $n^{p /(2 p+1)} \hat{\chi} \underset{\mathcal{F}, \mathcal{Q}_{n}}{\stackrel{p}{\rightarrow}} \chi_{\infty}$ where $\chi_{\infty}=\mathrm{cv}_{\alpha}\left(\overline{\text { bias }}_{\infty} / \sqrt{v_{\infty}}\right) \sqrt{v_{\infty}}$ with $\overline{\text { bias }} \infty$ and $\sqrt{v_{\infty}}$ given in (S22) and $\delta_{\infty}=z_{\beta}+z_{1-\alpha}$ if excess length is the criterion, $\delta_{\infty}=\arg \min _{\delta} \operatorname{cv}_{\alpha}\left(\frac{\omega_{\infty}(\delta)}{2 \omega_{\infty}^{\prime}(\delta)}-\right.$ $\left.\frac{\delta}{2}\right) \omega_{\infty}^{\prime}(\delta)$ if FLCI length is the criterion, and $\delta_{\infty}=\arg \min _{\delta}\left[\frac{1}{4}\left(\omega_{\infty}\left(\delta_{\infty}\right)-\delta_{\infty} \omega_{\infty}^{\prime}\left(\delta_{\infty}\right)\right)^{2}+\right.$ $\left.\omega_{\infty}^{\prime}(\delta)^{2}\right]$ if RMSE is the criterion.

Suppose, in addition, that each $\mathcal{Q}_{n}$ contains a distribution where the $u_{i}$ s are normal. If the FLCI criterion is used, then no other sequence of linear estimators $\tilde{L}$ can satisfy

$$
\liminf _{n \rightarrow \infty} \inf _{f \in \mathcal{F}, Q \in \mathcal{Q}_{n}} P_{f, Q}\left(L f \in\left\{\tilde{L} \pm n^{-p /(2 p+1)} \chi\right\}\right) \geq 1-\alpha
$$

with $\chi$ a constant with $\chi<\chi_{\infty}$. In addition, for any sequence of confidence sets $\mathcal{C}$ with $\liminf _{n \rightarrow \infty} \inf _{f \in \mathcal{F}, Q \in \mathcal{Q}_{n}} P_{f, Q}(L f \in \mathcal{C}) \geq 1-\alpha$, we have the following bound on the asymptotic efficiency improvement at any $f \in \mathcal{F}_{R D T, p}(0)$ :

$$
\liminf _{n \rightarrow \infty} \sup _{Q \in \mathcal{Q}_{n}} \frac{n^{p /(2 p+1)} E_{f, Q} \lambda(\mathcal{C})}{2 \chi_{\infty}} \geq \frac{(1-\alpha) 2^{2 p /(2 p+1)} E\left[\left(z_{1-\alpha}-Z\right)^{2 p /(2 p+1)} \mid Z \leq z_{1-\alpha}\right]}{\frac{4 p}{2 p+1} \inf _{\delta>0} \operatorname{cv}_{\alpha}(\delta /(4 p)) \delta^{-1 /(2 p+1)}}
$$

where $Z \sim \mathcal{N}(0,1)$.

If the excess length criterion is used with quantile $\beta$ (i.e. $\delta_{n}=z_{\beta}+z_{1-\alpha}$ ), then any one-sided $C I[\hat{c}, \infty)$ with

$$
\liminf _{n \rightarrow \infty} \inf _{f \in \mathcal{F}, Q \in \mathcal{Q}_{n}} P_{f, Q}(L f \in[\hat{c}, \infty)) \geq 1-\alpha
$$

must satisfy

$$
\liminf _{n \rightarrow \infty} \frac{\sup _{f \in \mathcal{F}, Q \in \mathcal{Q}_{n}} q_{f, Q, \beta}(L f-\hat{c})}{\sup _{f \in \mathcal{F}, Q \in \mathcal{Q}_{n}} q_{f, Q, \beta}\left(L f-\hat{c}_{\alpha, \delta_{n}}\right)} \geq 1
$$

and, for any $f \in \mathcal{F}_{R D T, p}(0)$,

$$
\liminf _{n \rightarrow \infty} \frac{\sup _{Q \in \mathcal{Q}_{n}} q_{f, Q, \beta}(L f-\hat{c})}{\sup _{Q \in \mathcal{Q}_{n}} q_{f, Q, \beta}\left(L f-\hat{c}_{\alpha, \delta_{n}}\right)} \geq \frac{2^{2 p /(2 p+1)}}{1+2 p /(2 p+1)} .
$$

To prove this theorem, we first prove a series of lemmas. To deal with the case where $\delta$ 
is chosen to optimize FLCI length or MSE, we will use the characterization of the optimal $\delta$ for these criteria from Donoho (1994), which is described at the beginning of Supplemental Appendix G. In particular, for $\rho_{A}$ and $\chi_{A, \alpha}$ given in Supplemental Appendix G, the $\delta$ that optimizes FLCI length is given by the $\delta$ that maximizes $\omega_{\tilde{\sigma}(\cdot), n}(\delta) \chi_{A, \alpha}(\delta) / \delta$, and the resulting FLCI half-length is given by $\sup _{\delta>0} \omega_{\tilde{\sigma}(\cdot), n}(\delta) \chi_{A, \alpha}(\delta) / \delta$. In addition, when $\delta$ is chosen to optimize FLCI length, $\chi_{\infty}$ in Theorem H.3 is given by $\sup _{\delta>0} \omega_{\infty}(\delta) \chi_{A, \alpha}(\delta) / \delta$, and $\delta_{\infty}$ maximizes this expression. If $\delta$ is chosen according to the MSE criterion, then $\delta$ maximizes $\omega_{\tilde{\sigma}(\cdot), n}(\delta) \sqrt{\rho_{A}(\delta)} / \delta$ and $\delta_{\infty}$ maximizes $\omega_{\infty}(\delta) \sqrt{\rho_{A}(\delta)} / \delta$.

Lemma H.2. For any constant B, the following holds. Under Assumption H.4,

$$
\lim _{n \rightarrow \infty} \sup _{\left\|\left(b_{+}, b_{-}, d_{+}, d_{-}\right)\right\| \leq B}\left|G_{n}\left(b_{+}, b_{-}, d_{+}, d_{-}\right)-G_{\infty}\left(b_{+}, b_{-}, d_{+}, d_{-}\right)\right|=0 .
$$

Under Assumption H.3,

$$
\sup _{\left\|\left(b_{+}, b_{-}, d_{+}, d_{-}\right)\right\| \leq B}\left|G_{n}\left(b_{+}, b_{-}, d_{+}, d_{-}\right)-G_{\infty}\left(b_{+}, b_{-}, d_{+}, d_{-}\right)\right| \underset{\mathcal{F}, \mathcal{Q}_{n}}{\stackrel{p}{\longrightarrow}} 0 .
$$

Proof. Define $\tilde{G}_{n}^{+}\left(b_{+}, d_{+}\right)=\frac{1}{n h_{n}} \sum_{i=1}^{n} k_{1}^{+}\left(x_{i} / h_{n} ; b_{+}, d_{+}\right)^{2}$, and define $\tilde{G}_{n}^{-}$analogously. Also, $\tilde{G}_{\infty}^{+}\left(b_{+}, d_{+}\right)=p_{X,+}(0) \int_{0}^{\infty} k_{1}^{+}\left(u ; b_{+}, d_{+}\right)^{2} d u$, with $G_{\infty}^{-}$defined analogously. For each $\left(b_{+}, d_{+}\right)$, $\tilde{G}_{n}\left(b_{+}, d_{+}\right) \rightarrow G_{\infty}\left(b_{+}, d_{+}\right)$by Assumption H.1. To show uniform convergence, first note that, for some constant $K_{1}$, the support of $k_{1}^{+}\left(\cdot ; b_{+}, d_{+}\right)$is bounded by $K_{1}$ uniformly over $\left\|\left(b_{+}, d_{+}\right)\right\| \leq B$ and similarly for $k_{1}^{-}\left(\cdot ; b_{-}, d_{-}\right)$. Thus, for any $\left(b_{+}, d_{+}\right)$and $\left(\bar{b}_{+}, \bar{d}_{+}\right)$,

$\left|G_{n}^{+}\left(b_{+}, d_{+}\right)-G_{n}^{+}\left(\bar{b}_{+}, \bar{d}_{+}\right)\right| \leq\left[\frac{1}{n h_{n}} \sum_{i=1}^{n} 1\left(\left|x_{i} / h_{n}\right| \leq K_{1}\right)\right] \sup _{|u| \leq K_{1}}\left|k_{1}^{+}\left(u ; b_{+}, d_{+}\right)-k_{1}^{+}\left(u ; \bar{b}_{+}, \bar{d}_{+}\right)\right|$.

Since the term in brackets converges to a finite constant by Assumption H.1 and $k_{1}^{+}$is Lipschitz continuous on any bounded set, it follows that there exists a constant $K_{2}$ such that $\left|G_{n}^{+}\left(b_{+}, d_{+}\right)-G_{n}^{+}\left(\bar{b}_{+}, \bar{d}_{+}\right)\right| \leq K_{2}\left\|\left(b_{+}, d_{+}\right)-\left(\bar{b}_{+}, \bar{d}_{+}\right)\right\|$for all $n$. Using this and applying pointwise convergence of $G_{n}^{+}\left(b_{+}, d_{+}\right)$on a small enough grid along with uniform continuity of $G_{\infty}\left(b_{+}, d_{+}\right)$on compact sets, it follows that

$$
\lim _{n \rightarrow \infty} \sup _{\left\|\left(b_{+}, b_{-}, d_{+}, d_{-}\right)\right\| \leq B}\left|\tilde{G}_{n}\left(b_{+}, d_{+}\right)-\tilde{G}_{\infty}\left(b_{+}, d_{+}\right)\right|=0,
$$


and similar arguments give the same statement for $\tilde{G}_{n}^{-}$and $\tilde{G}_{\infty}^{-}$. Under Assumption H.4,

$$
\begin{aligned}
& \left|G_{n}\left(b_{+}, b_{-}, d_{+}, d_{-}\right)-\left[\tilde{G}_{n}\left(b_{+}, d_{+}\right) \tilde{\sigma}_{+}^{2}(0)+\tilde{G}_{n}\left(b_{-}, d_{-}\right) \tilde{\sigma}_{-}^{2}(0)\right]\right| \leq \\
& \bar{k} \cdot\left[\frac{1}{n h_{n}} \sum_{i=1}^{n} 1\left(\left|x_{i} / h_{n}\right| \leq K_{1}\right)\right]\left[\sup _{0<x \leq K_{1} h_{n}}\left|\tilde{\sigma}_{+}^{2}(0)-\tilde{\sigma}_{+}^{2}(x)\right|+\sup _{-K_{1} h_{n} \leq x<0}\left|\tilde{\sigma}_{-}^{2}(0)-\tilde{\sigma}_{-}^{2}(x)\right|\right]
\end{aligned}
$$

where $\bar{k}$ is an upper bound for $\left|k_{1}^{+}(x)\right|$ and $\left|k_{1}^{-}(x)\right|$. This converges to zero by left- and rightcontinuity of $\tilde{\sigma}$ at 0 . The result then follows since $G_{\infty}\left(b_{+}, b_{-}, d_{+}, d_{-}\right)=\tilde{\sigma}_{+}^{2}(0) \tilde{G}_{\infty}^{+}\left(b_{+}, d_{+}\right)+$ $\tilde{\sigma}_{-}^{2}(0) \tilde{G}_{\infty}^{-}\left(b_{-}, d_{-}\right)$. Under Assumption H.3, we have $G_{n}\left(b_{+}, b_{-}, d_{+}, d_{-}\right)=\tilde{G}_{n}^{+}\left(b_{+}, d_{+}\right) \hat{\sigma}_{+}^{2}+$ $\tilde{G}_{n}^{+}\left(b_{-}, d_{-}\right) \hat{\sigma}_{-}^{2}$, and the result follows from uniform convergence in probability of $\hat{\sigma}_{+}^{2}$ and $\hat{\sigma}_{-}^{2}$ to $\tilde{\sigma}_{+}^{2}(0)$ and $\tilde{\sigma}_{-}^{2}(0)$.

Lemma H.3. Under Assumption H.4, $\left\|\left(\tilde{b}_{+, n}, \tilde{b}_{-, n}, \tilde{d}_{+, n}, \tilde{d}_{-, n}\right)\right\| \leq B$ for some constant $B$ and $n$ large enough. Under Assumption H.3, the same statement holds with probability approaching one uniformly over $\mathcal{F}, \mathcal{Q}_{n}$.

Proof. Let $\mathcal{A}(x, b, d)=b+\sum_{i=1}^{p-1} d\left(x / h_{n}\right)^{j}$, where $d=\left(d_{1}, \ldots, d_{p-1}\right)$. Note $G_{n}\left(b_{+}, b_{-}, d_{+}, d_{-}\right)$ is bounded from below by $1 / \sup _{|x| \leq h_{n}} \tilde{\sigma}^{2}(x)$ times

$$
\begin{aligned}
& \frac{1}{n h_{n}} \sum_{i: 0<x_{i} \leq h_{n}}\left(\left|\mathcal{A}\left(x_{i}, b_{+}, d_{+}\right)\right|-C\right)_{+}^{2}+\frac{1}{n h_{n}} \sum_{i:-h_{n} \leq x_{i}<0}\left(\left|\mathcal{A}\left(x_{i}, b_{-}, d_{-}\right)\right|-C\right)_{+}^{2} \\
& \quad \geq \frac{1}{4 n h_{n}} \sum_{i: 0<x_{i} \leq h_{n}}\left[\mathcal{A}\left(x_{i}, b_{+}, d_{+}\right)^{2}-4 C^{2}\right]+\frac{1}{4 n h_{n}} \sum_{i:-h_{n} \leq x_{i}<0}\left[\mathcal{A}\left(x_{i}, b_{-}, d_{-}\right)^{2}-4 C^{2}\right]
\end{aligned}
$$

(the inequality follows since, for any $s \geq 2 C,(s-C)^{2} \geq s^{2} / 4 \geq s^{2} / 4-C^{2}$ and, for $\left.2 C \geq s \geq C,(s-C)^{2} \geq 0 \geq s^{2} / 4-C^{2}\right)$. Note that, for any $B>0$

$$
\begin{aligned}
\max _{\max \left\{\left|b_{+}\right|,\left|d_{+, 1}\right|, \ldots,\left|d_{+, p-1}\right|\right\} \geq B} & \frac{1}{4 n h_{n}} \sum_{i: 0<x_{i} \leq h_{n}} \mathcal{A}\left(x_{i}, b_{+}, d_{+}\right)^{2} \\
=B^{2} & \inf _{\max \left\{\left|b_{+}\right|,\left|d_{+, 1}\right|, \ldots,\left|d_{+, p-1}\right|\right\} \geq 1} \frac{1}{4 n h_{n}} \sum_{i: 0<x_{i} \leq h_{n}} \mathcal{A}\left(x_{i}, b_{+}, d_{+}\right)^{2} \\
& \rightarrow \frac{p_{X,+}(0)}{4} B^{2}{\max \left\{\left|b_{+}\right|,\left|d_{+, 1}\right|, \ldots,\left|d_{+, p-1}\right|\right\} \geq 1}_{0} \int_{0}^{\infty}\left(b_{+}+\sum_{i=1}^{p-1} d_{+, j} u^{j}\right)^{2} d u
\end{aligned}
$$

and similarly for the term involving $\mathcal{A}\left(x_{i}, b_{-}, d_{-}\right)$(the convergence follows since the infimum is taken on the compact set where $\max \left\{\left|b_{+}\right|,\left|d_{+, 1}\right|, \ldots,\left|d_{+, p-1}\right|\right\}=1$ ). Combining 
this with the previous display and the fact that $\frac{1}{n h} \sum_{i:\left|x_{i}\right| \leq h_{n}} C^{2}$ converges to a finite constant, it follows that, for some $\eta>0$, inf $\max \left\{\left|b_{+}\right|,\left|d_{+, 1}\right|, \ldots,\left|d_{+, p-1}\right|\right\} \geq B G_{n}\left(b_{+}, b_{-}, d_{+}, d_{-}\right) \geq\left(B^{2} \eta-\right.$ $\left.\eta^{-1}\right) / \sup _{|x| \leq h_{n}} \tilde{\sigma}^{2}(x)$ for large enough $n$. Let $K$ be such that $G_{\infty}\left(\tilde{b}_{+, \infty}, \tilde{b}_{-, \infty}, \tilde{d}_{+, \infty}, \tilde{d}_{-, \infty}\right) \leq$ $K / 2$ and $\max \left\{\tilde{\sigma}_{+}^{2}(0), \tilde{\sigma}_{-}^{2}(0)\right\} \leq K / 2$. Under Assumption H.4, $G_{n}\left(\tilde{b}_{+, \infty}, \tilde{b}_{-, \infty}, \tilde{d}_{+, \infty}, \tilde{d}_{-, \infty}\right)<$ $K$ and $\sup _{|x| \leq h_{n}} \tilde{\sigma}^{2}(x) \leq K$ for large enough $n$. Under Assumption H.3, $G_{n}\left(\tilde{b}_{+, \infty}, \tilde{b}_{-, \infty}, \tilde{d}_{+, \infty}\right.$, $\left.\tilde{d}_{-, \infty}\right)<K$ and $\sup _{|x| \leq h_{n}} \tilde{\sigma}^{2}(x) \leq K$ with probability approaching one uniformly over $\mathcal{F}, \mathcal{Q}_{n}$. Let $B$ be large enough so that $\left(B^{2} \eta-\eta^{-1}\right) / K>K$. Then, when $G_{n}\left(\tilde{b}_{+, \infty}, \tilde{b}_{-, \infty}, \tilde{d}_{+, \infty}, \tilde{d}_{-, \infty}\right) \leq$ $K$ and $\sup _{|x| \leq h_{n}} \tilde{\sigma}^{2}(x) \leq K,\left(\tilde{b}_{+, \infty}, \tilde{b}_{-, \infty}, \tilde{d}_{+, \infty}, \tilde{d}_{-, \infty}\right)$ will give a lower value of $G_{n}$ than any $\left(b_{+}, b_{-}, d_{+}, d_{-}\right)$with $\max \left\{\left|b_{+}\right|,\left|d_{+, 1}\right|, \ldots,\left|d_{+, p-1}\right|,\left|b_{-}\right|,\left|d_{-, 1}\right|, \ldots,\left|d_{-, p-1}\right|\right\} \geq B$. The result follows from the fact that the max norm on $\mathbb{R}^{2 p}$ is bounded from below by a constant times the Euclidean norm.

Lemma H.4. If Assumption H.4 holds and $\tilde{b}_{n} \rightarrow \tilde{b}_{\infty}$, then $\left(\tilde{b}_{+, n}, \tilde{b}_{-, n}, \tilde{d}_{+, n}, \tilde{d}_{-, n}\right) \rightarrow\left(\tilde{b}_{+, \infty}\right.$, $\left.\tilde{b}_{-, \infty}, \tilde{d}_{+, \infty}, \tilde{d}_{-, \infty}\right)$. If Assumption H.3 holds and $\tilde{b}_{n} \underset{\mathcal{F}, \mathcal{Q}_{n}}{\stackrel{p}{\rightarrow}} \tilde{b}_{\infty}>0,\left(\tilde{b}_{+, n}, \tilde{b}_{-, n}, \tilde{d}_{+, n}, \tilde{d}_{-, n}\right) \underset{\mathcal{F}, \mathcal{Q}_{n}}{\stackrel{p}{\rightarrow}}$ $\left(\tilde{b}_{+, \infty}, \tilde{b}_{-, \infty}, \tilde{d}_{+, \infty}, \tilde{d}_{-, \infty}\right)$.

Proof. By Lemma H.3, $B$ can be chosen so that $\left\|\left(\tilde{b}_{+, n}, \tilde{b}_{-, n}, \tilde{d}_{+, n}, \tilde{d}_{-, n}\right)\right\| \leq B$ for large enough $n$ under Assumption H.4 and $\left\|\left(\tilde{b}_{+, n}, \tilde{b}_{-, n}, \tilde{d}_{+, n}, \tilde{d}_{-, n}\right) \leq B\right\|$ with probability one uniformly over $\mathcal{F}, \mathcal{Q}_{n}$ under Assumption H.3. The result follows from Lemma H.2, continuity of $G_{\infty}$ and the fact that $G_{\infty}$ has a unique minimizer.

Lemma H.5. If Assumption H.4 holds and $\tilde{b}_{n} \rightarrow \tilde{b}_{\infty}>0$, then $\omega_{n}^{-1}\left(n^{p /(2 p+1)} \tilde{b}_{n}\right) \rightarrow \omega_{\infty}^{-1}\left(\tilde{b}_{\infty}\right)$. If Assumption H.3 holds and $\tilde{b}_{n} \underset{\mathcal{F}, \mathcal{Q}_{n}}{\stackrel{p}{\rightarrow}} b_{\infty}>0$, then $\omega_{n}^{-1}\left(n^{p /(2 p+1)} \tilde{b}_{n}\right) \underset{\mathcal{F}, \mathcal{Q}_{n}}{\stackrel{p}{\rightarrow}} \omega_{\infty}^{-1}\left(\tilde{b}_{\infty}\right)$.

Proof. The result is immediate from Lemmas H.2 and H.4.

Lemma H.6. Under Assumption H.4, we have, for any $\bar{\delta}>0$,

$$
\sup _{0<\delta \leq \bar{\delta}}\left|n^{p /(2 p+1)} \omega_{n}(\delta)-\omega_{\infty}(\delta)\right| \rightarrow 0
$$

Under Assumption H.3, we have, for any $\bar{\delta}>0$,

$$
\sup _{0<\delta \leq \bar{\delta}}\left|n^{p /(2 p+1)} \omega_{n}(\delta)-\omega_{\infty}(\delta)\right| \underset{\mathcal{F}, \mathcal{Q}_{n}}{\stackrel{p}{\rightarrow}} 0
$$

Proof. The first statement is immediate from Lemma H.5 and Lemma G.1 (with $n^{p /(2 p+1)} \omega_{n}$ playing the role of $\omega_{n}$ in that lemma). For the second claim, note that, if $\left|\hat{\sigma}_{+}-\sigma_{+}(0)\right| \leq \eta$ and $\left|\hat{\sigma}_{-}-\sigma_{-}(0)\right| \leq \eta, \omega_{n, \underline{\sigma}(\cdot)}(\delta) \leq \omega_{\tilde{\sigma}(\cdot), n}(\delta) \leq \omega_{n, \bar{\sigma}(\cdot)}(\delta)$, where $\underline{\sigma}(x)=\left(\sigma_{+}(0)-\eta\right) 1(x>$ 
$0)+\left(\sigma_{-}(0)-\eta\right) 1(x<0)$ and $\bar{\sigma}(x)$ is defined similarly. Applying the first statement in the lemma and the fact that $\left|\hat{\sigma}_{+}-\sigma_{+}(0)\right| \leq \eta$ and $\left|\hat{\sigma}_{-}-\sigma_{-}(0)\right| \leq \eta$ with probability approaching one uniformly over $\mathcal{F}, \mathcal{Q}_{n}$, it follows that, for any $\varepsilon>0$, we will have

$$
\omega_{\underline{\sigma}_{+}(0), \underline{\sigma}_{-}(0), \infty}(\delta)-\varepsilon \leq n^{p /(2 p+1)} \omega_{n}(\delta) \leq \omega_{\bar{\sigma}_{+}(0), \bar{\sigma}_{-}(0), \infty}(\delta)+\varepsilon
$$

for all $0<\delta<\bar{\delta}$ with probability approaching one uniformly over $\mathcal{F}, \mathcal{Q}_{n}$. By making $\eta$ and $\varepsilon$ small, both sides can be made arbitrarily close to $\omega_{\infty}(\delta)=\omega_{\infty, \sigma_{+}(0), \sigma_{-}(0)}(\delta)$.

Lemma H.7. Let $r$ denote $\sqrt{\rho_{A}}$ or $\chi_{A, \alpha}$. Under Assumption H.4,

$$
\sup _{\delta>0} n^{p /(2 p+1)} \omega_{n}(\delta) r(\delta / 2) / \delta \rightarrow \sup _{\delta>0} \omega_{\infty}(\delta) r(\delta / 2) / \delta
$$

Let $\delta_{n}$ minimize the left-hand side of the above display, and let $\delta^{*}$ minimize the right-hand side. Then $\delta_{n} \rightarrow \delta^{*}$ under Assumption H.4 and $\delta_{n} \underset{\mathcal{F}, \mathcal{Q}_{n}}{\stackrel{p}{\rightarrow}} \delta^{*}$ under Assumption H.3. In addition, for any $0<\alpha<1$ and $Z$ a standard normal variable,

$$
\lim _{n \rightarrow \infty}(1-\alpha) E\left[n^{p /(2 p+1)} \omega_{n}\left(2\left(z_{1-\alpha}-Z\right)\right) \mid Z \leq z_{1-\alpha}\right]=(1-\alpha) E\left[\omega_{\infty}\left(2\left(z_{1-\alpha}-Z\right)\right) \mid Z \leq z_{1-\alpha}\right]
$$

Proof. All the statements are immediate from Lemmas H.6 and G.2 except for the statement that $\delta_{n} \underset{\mathcal{F}, \mathcal{Q}_{n}}{\stackrel{p}{\rightarrow}} \delta^{*}$ under Assumption H.3. The statement that $\delta_{n} \underset{\mathcal{F}, \mathcal{Q}_{n}}{\stackrel{p}{\rightarrow}} \delta^{*}$ under Assumption H.3 follows by using Lemma H.6 and analogous arguments to those in Lemma G.2 to show that there exist $0<\underline{\delta}<\bar{\delta}$ such that $\delta_{n} \in[\underline{\delta}, \bar{\delta}]$ with probability approaching on uniformly in $\mathcal{F}, \mathcal{Q}_{n}$, and that $\sup _{\delta \in[\underline{\delta}, \bar{\delta}]}\left|n^{p /(2 p+1)} \omega_{n}(\delta) r(\delta / 2) / \delta-\omega(\delta) r(\delta / 2) / \delta\right| \underset{\mathcal{F}, \mathcal{Q}_{n}}{\stackrel{p}{\rightarrow}} 0$.

Lemma H.8. Under Assumptions H.1 and H.2, the following hold. If Assumption H.4 holds and $\tilde{b}_{n}$ is a deterministic sequence with $\tilde{b}_{n} \rightarrow \tilde{b}_{\infty}>0$, then

$$
\begin{aligned}
& \sup _{x}\left|k_{\tilde{\sigma}(\cdot)}^{+}\left(x ; \tilde{b}_{+, n}, \tilde{d}_{+, n}\right)-k_{\tilde{\sigma}_{+}(0)}^{+}\left(x ; \tilde{b}_{+, \infty}, \tilde{d}_{+, \infty}\right)\right| \rightarrow 0, \\
& \sup _{x}\left|k_{\tilde{\sigma}(\cdot)}^{-}\left(x ; \tilde{b}_{-, n}, \tilde{d}_{-, n}\right)-k_{\tilde{\sigma}_{-}(0)}^{-}\left(x ; \tilde{b}_{-, \infty}, \tilde{d}_{-, \infty}\right)\right| \rightarrow 0 .
\end{aligned}
$$

If Assumption H.3 holds and $\tilde{b}_{n}$ is a random sequence with $\tilde{b}_{n} \underset{\mathcal{F}, \mathcal{Q}_{n}}{\stackrel{p}{\rightarrow}} \tilde{b}_{\infty}>0$, then

$$
\begin{aligned}
& \sup _{x}\left|k_{\tilde{\sigma}(\cdot)}^{+}\left(x ; \tilde{b}_{+, n}, \tilde{d}_{+, n}\right)-k_{\tilde{\sigma}_{+}(0)}^{+}\left(x ; \tilde{b}_{+, \infty}, \tilde{d}_{+, \infty}\right)\right| \underset{\mathcal{F}, \mathcal{Q}_{n}}{\stackrel{p}{\rightarrow}} 0 \\
& \sup _{x}\left|k_{\tilde{\sigma}(\cdot)}^{-}\left(x ; \tilde{b}_{-, n}, \tilde{d}_{-, n}\right)-k_{\tilde{\sigma}_{-}(0)}^{-}\left(x ; \tilde{b}_{-, \infty}, \tilde{d}_{-, \infty}\right)\right| \underset{\mathcal{F}, \mathcal{Q}_{n}}{\stackrel{p}{\longrightarrow}} 0
\end{aligned}
$$


Proof. Note that

$$
\begin{aligned}
\left|k_{\tilde{\sigma}(\cdot)}^{+}\left(x ; \tilde{b}_{+, n}, \tilde{d}_{+, n}\right)-k_{\tilde{\sigma}_{+}(0)}^{+}\left(x ; \tilde{b}_{+, \infty}, \tilde{d}_{+, \infty}\right)\right| & \leq\left|k_{\tilde{\sigma}(\cdot)}^{+}\left(x ; \tilde{b}_{+, n}, \tilde{d}_{+, n}\right)-k_{\tilde{\sigma}_{+}(0)}^{+}\left(x ; \tilde{b}_{+, n}, \tilde{d}_{+, n}\right)\right| \\
& +\left|k_{\tilde{\sigma}_{+}(0)}^{+}\left(x ; \tilde{b}_{+, n}, \tilde{d}_{+, n}\right)-k_{\tilde{\sigma}_{+}(0)}^{+}\left(x ; \tilde{b}_{+, \infty}, \tilde{d}_{+, \infty}\right)\right| .
\end{aligned}
$$

Under Assumption H.4, the first term is, for large enough $n$, bounded by a constant times $\sup _{0<x<h_{n} K}\left|\tilde{\sigma}^{-2}(x)-\tilde{\sigma}_{+}^{-2}(0)\right|$, where $K$ is bound on the support of $k_{1}^{+}\left(\cdot ; b_{+}, d_{+}\right)$over $b_{+}, d_{+}$in a neighborhood of $\tilde{b}_{+, \infty}, \tilde{d}_{+, \infty}$. This converges to zero by Assumption H.4. The second term converges to zero by Lipschitz continuity of $k_{\tilde{\sigma}_{+}(0)}^{+}$. Under Assumption H.3, the first term is bounded by a constant times $\left|\hat{\sigma}_{+}^{-2}-\tilde{\sigma}_{+}(0)\right|$, which converges in probability to zero uniformly over $\mathcal{F}, \mathcal{Q}_{n}$ by assumption. The second term converges in probability to zero uniformly over $\mathcal{F}, \mathcal{Q}_{n}$ by Lipschitz continuity of $k_{\tilde{\sigma}_{+}(0)}^{+}$. Similar arguments apply to $k_{\tilde{\sigma}(\cdot)}^{-}$in both cases.

Lemma H.9. Under Assumptions H.1 and H.2, the following holds. Let $\hat{L}$ denote the estimator $\hat{L}_{\delta_{n}, \tilde{\sigma}(\cdot)}$ where $\tilde{\sigma}(\cdot)$ satisfies Assumption $H .4$ and $\delta_{n}=\omega_{\tilde{\sigma}(\cdot), n}^{-1}\left(2 n^{-p /(2 p+1)} \tilde{b}_{n}\right)$ where $\tilde{b}_{n}$ is a deterministic sequence with $\tilde{b}_{n} \rightarrow \tilde{b}_{\infty}$. Let $\overline{\text { bias }}_{n}$ and $\tilde{v}_{n}$ denote the corresponding worst-case bias and variance formulas. Let $\hat{L}^{*}$ denote the estimator $\hat{L}_{\delta_{n}^{*}, \tilde{\sigma}(\cdot)}$ where $\tilde{\sigma}^{*}(\cdot)=$ $\hat{\sigma}_{+} 1(x>0)+\hat{\sigma}_{-} 1(x<0)$ satisfies Assumption H.3 with the same value of $\tilde{\sigma}_{+}(0)$ and $\tilde{\sigma}_{-}(0)$ and $\delta_{n}^{*}=\omega_{\tilde{\sigma}(\cdot), n}^{-1}\left(2 n^{-p /(2 p+1)} \tilde{b}_{n}^{*}\right)$ where $\tilde{b}_{n}^{*} \underset{\mathcal{F}, \mathcal{Q}_{n}}{\stackrel{p}{\rightarrow}} \tilde{b}_{\infty}$. Let $\overrightarrow{\text { bias }}_{n}^{*}$ and $\tilde{v}_{n}^{*}$ denote the corresponding worst-case bias and variance formulas. Then

$$
n^{p /(2 p+1)}\left(\hat{L}-\hat{L}^{*}\right) \underset{\mathcal{F}, \mathcal{Q}_{n}}{\stackrel{p}{\rightarrow}} 0, \quad n^{p /(2 p+1)}\left({\overrightarrow{b i a s_{n}}}-{\overrightarrow{b i a s_{n}}}_{n}^{*} \underset{\mathcal{F}, \mathcal{Q}_{n}}{\stackrel{p}{\rightarrow}} 0, \quad \frac{\tilde{v}_{n}}{\tilde{v}_{n}^{*}} \underset{\mathcal{F}, \mathcal{Q}_{n}}{\stackrel{p}{\rightarrow}} 1 .\right.
$$

Proof. We have

$$
\hat{L}=\frac{1}{n h_{n}} \sum_{i=1}^{n} w_{n}\left(x_{i} / h_{n}\right) y_{i}=\frac{1}{n h_{n}} \sum_{i=1}^{n} w_{n}\left(x_{i} / h_{n}\right) f\left(x_{i}\right)+\frac{1}{n h_{n}} \sum_{i=1}^{n} w_{n}\left(x_{i} / h_{n}\right) u_{i}
$$

where $w_{n}(u)=\frac{k_{\tilde{\sigma}(\cdot)}^{+}\left(u ; \tilde{b}_{+, n}, \tilde{d}_{+, n}\right)}{\frac{1}{n h_{n}} \sum_{j=1}^{n} k_{\tilde{\sigma}(\cdot)}^{+}\left(x_{j} / h_{n} ; \tilde{b}_{+, n}, \tilde{d}_{+, n}\right)}$ for $u>0$ and similarly with $k_{\tilde{\sigma}(\cdot)}^{+}$replaced by $k_{\tilde{\sigma}(\cdot)}^{-}$ for $u<0$ (here, $\tilde{d}_{+, n}, \tilde{d}_{-, n}, \tilde{b}_{+, n}$ and $\tilde{b}_{-, n}$ are the coefficients in the solution to the inverse modulus problem defined above). Similarly, $\hat{L}^{*}$ takes the same form with $w_{n}$ replaced by $w_{n}^{*}(u)=\frac{k_{\tilde{\sigma}^{*}(\cdot)}^{+}\left(u ; \tilde{b}_{n}^{*}, \tilde{d}_{n}^{*}\right)}{\frac{1}{n h_{n}} \sum_{j=1}^{n} k_{\tilde{\sigma}^{*}(\cdot)}^{+}\left(x_{j} / h_{n}, \tilde{b}_{n}^{*}, \tilde{d}_{n}^{*}\right)}$ for $u>0$ and similarly for $u<0$ (with $\tilde{d}_{+, n}^{*}, \tilde{d}_{-, n}^{*}, \tilde{b}_{+, n}^{*}$ and $\tilde{b}_{-, n}^{*}$ the coefficients in the solution to the corresponding inverse modulus problem). Let $w_{\infty}(u)=\frac{k_{\tilde{\sigma}(\cdot)}^{+}\left(u ; \tilde{b}_{n}^{*}, \tilde{d}_{n}^{*}\right)}{p_{X,+}(0) \int k_{\tilde{\sigma}(\cdot)}^{+}\left(u ; \tilde{b}_{\infty}, \tilde{d}_{\infty}\right) d u}$ Note that, by Lemma H.8, $\sup _{u}\left|w_{n}(u)-w_{\infty}(u)\right| \rightarrow 0$ and 
$\sup _{u}\left|w_{n}^{*}(u)-w_{\infty}(u)\right| \underset{\mathcal{F}, \mathcal{Q}_{n}}{\stackrel{p}{\rightarrow}} 0$.

We have

$$
\hat{L}-\hat{L}^{*}=\frac{1}{n h_{n}} \sum_{i=1}^{n}\left[w_{n}\left(x_{i} / h_{n}\right)-w_{n}^{*}\left(x_{i} / h_{n}\right)\right] r\left(x_{i}\right)+\frac{1}{n h_{n}} \sum_{i=1}^{n}\left[w_{n}\left(x_{i} / h_{n}\right)-w_{n}^{*}\left(x_{i} / h_{n}\right)\right] u_{i}
$$

where $f(x)=\sum_{j=0}^{p-1} f_{+}^{(j)}(0) x^{j} 1(x>0) / j !+\sum_{j=0}^{p-1} f_{-}^{(j)}(0) x^{j} 1(x<0) / j !+r(x)$ and we use the fact that $\sum_{i=1}^{n} w_{n}\left(x_{i} / h_{n}\right) x_{i}^{j}=\sum_{i=1}^{n} w_{n}^{*}\left(x_{i} / h_{n}\right) x_{i}^{j}$ for $j=0, \ldots, p-1$. Let $B$ be such that, with probability approaching one, $w_{n}(x)=w_{n}^{*}(x)=0$ for all $x$ with $|x| \geq B$. The first term is bounded by

$$
\frac{C}{n h_{n}} \sum_{i=1}^{n}\left|w_{n}\left(x_{i} / h_{n}\right)-w_{n}^{*}\left(x_{i} / h_{n}\right)\right| \cdot\left|x_{i}\right|^{p} \leq \sup _{x}\left|w_{n}(x)-w_{n}^{*}(x)\right| B h_{n}^{p} \frac{C}{n h_{n}} \sum_{i=1}^{n} 1\left(\left|x_{i} / h_{n}\right| \leq B\right) .
$$

It follows from Lemma H.8 that $\sup _{x}\left|w_{n}(x)-w_{n}^{*}(x)\right| \underset{\mathcal{F}, \mathcal{Q}_{n}}{\stackrel{p}{\rightarrow}} 0$. Also, $\frac{1}{n h_{n}} \sum_{i=1}^{n} 1\left(\left|x_{i} / h_{n}\right| \leq\right.$ $B)$ converges to a finite constant by Assumption H.1. Thus, the above display converges uniformly in probability to zero when scaled by $n^{p /(2 p+1)}=h_{n}^{-p}$.

For the last term in $\hat{L}-\hat{L}^{*}$, scaling by $n^{p /(2 p+1)}$ gives

$$
\frac{1}{\sqrt{n h_{n}}} \sum_{i=1}^{n}\left[w_{n}\left(x_{i} / h_{n}\right)-w_{\infty}\left(x_{i} / h_{n}\right)\right] u_{i}-\frac{1}{\sqrt{n h_{n}}} \sum_{i=1}^{n}\left[w_{n}^{*}\left(x_{i} / h_{n}\right)-w_{\infty}\left(x_{i} / h_{n}\right)\right] u_{i}
$$

The first term has mean zero and variance $\frac{1}{n h} \sum_{i=1}^{n}\left[w_{n}\left(x_{i} / h_{n}\right)-w_{\infty}\left(x_{i} / h_{n}\right)\right]^{2} \sigma^{2}\left(x_{i}\right)$ which is bounded by $\left\{\sup _{u}\left[w_{n}(u)-w_{\infty}(u)\right]^{2}\right\}\left[\sup _{|x| \leq B h_{n}} \sigma^{2}(x)\right] \frac{1}{n h} \sum_{i=1}^{n} 1\left(\left|x_{i} / h_{n}\right| \leq B\right) \rightarrow 0$. Let $c_{n,+}=\frac{\hat{\sigma}_{+}^{2}}{n h_{n}} \sum_{i=1}^{n} k_{\tilde{\sigma}^{*}(\cdot)}\left(x_{i} / h_{n} ; \tilde{b}_{+, n}^{*}, \tilde{d}_{+, n}^{*}\right)$ and $c_{\infty,+}=\tilde{\sigma}_{+}^{2}(0) p_{X,+}(0) \int k_{\tilde{\sigma}^{*}(\cdot)}\left(u ; \tilde{b}_{\infty}, \tilde{d}_{\infty}\right)$ so that $c_{n,+} \underset{\mathcal{F}, \mathcal{Q}_{n}}{\stackrel{p}{\rightarrow}} c_{\infty,+}$, and define $c_{n,-}$ and $c_{\infty,-}$ analogously. With this notation, we have, for $x_{i}>0$,

$$
w_{n}^{*}\left(x_{i} / h_{n}\right)=c_{n,+}^{-1} \hat{\sigma}_{+}^{2} k_{\tilde{\sigma}^{*}(\cdot)}\left(x_{i} / h_{n} ; \tilde{b}_{+, n}^{*}, \tilde{d}_{+, n}^{*}\right)=c_{n,+}^{-1} h_{+}\left(x_{i} / h_{n} ; \tilde{b}_{+, n}^{*}, \tilde{d}_{+, n}^{*}\right)
$$

and $w_{\infty}(u)=c_{\infty,+}^{-1} h_{+}\left(x_{i} / h_{n} ; \tilde{b}_{+, \infty}, \tilde{d}_{+, \infty}\right)$ where

$$
h_{+}\left(u ; b_{+}, d_{+}\right)=\left(b_{+}+\sum_{j=1}^{p-1} d_{+, j} u^{j}-C|u|^{p}\right)_{+}-\left(b_{+}+\sum_{j=1}^{p-1} d_{+, j} u^{j}+C|u|^{p}\right)_{-} .
$$


Thus,

$$
\begin{aligned}
& \frac{1}{\sqrt{n h}} \sum_{i=1}^{n}\left[w_{n}^{*}\left(x_{i} / h_{n}\right)-w_{\infty}\left(x_{i} / h_{n}\right)\right] 1\left(x_{i}>0\right) u_{i} \\
&=\frac{c_{n,+}^{-1}}{\sqrt{n h}} \sum_{i=1}^{n}\left[h_{+}\left(u ; \tilde{b}_{+, n}, \tilde{d}_{+, n}\right)-h_{+}\left(u ; \tilde{b}_{+, \infty}, \tilde{d}_{+, \infty}\right)\right] 1\left(x_{i}>0\right) u_{i} \\
&+\frac{\left(c_{n,+}^{-1}-c_{n, \infty}^{-1}\right)}{\sqrt{n h}} \sum_{i=1}^{n} h_{+}\left(u ; \tilde{b}_{+, \infty}, \tilde{d}_{+, \infty}\right) 1\left(x_{i}>0\right) u_{i} .
\end{aligned}
$$

The last term converges to zero uniformly in probability by Slutsky's Theorem. The first term can be written as $c_{n,+}^{-1}$ times the sum of

$$
\begin{aligned}
\frac{1}{\sqrt{n h}} \sum_{i=1}^{n}\left[\left(\tilde{b}_{+, n}^{*}+\sum_{j=1}^{p-1} \tilde{d}_{+, n, j}^{*}\left(\frac{x_{i}}{h_{n}}\right)^{j}-C\left|\frac{x_{i}}{h_{n}}\right|^{p}\right)_{+}\right. & \left.-\left(\tilde{b}_{+, \infty}+\sum_{j=1}^{p-1} \tilde{d}_{+, \infty, j}\left(\frac{x_{i}}{h_{n}}\right)^{j}-C\left|\frac{x_{i}}{h_{n}}\right|^{p}\right)\right] u_{+}
\end{aligned}
$$

and a corresponding term with $(\cdot)_{+}$replaced by $(\cdot)_{-}$, which can be dealt with using similar arguments. Letting $A\left(b_{+}, d_{+}\right)=\left\{u: b_{+}+\sum_{j=1}^{p-1} d_{+, j} u^{j}-C|u|^{p} \geq 0\right\}$, the above display is equal to

$$
\begin{aligned}
& \frac{1}{\sqrt{n h}} \sum_{i=1}^{n}\left(\tilde{b}_{+, n}^{*}-\tilde{b}_{+, \infty}+\sum_{j=1}^{p-1}\left(\tilde{d}_{+, n, j}^{*}-\tilde{d}_{+, \infty, j}\right)\left(\frac{x_{i}}{h_{n}}\right)^{j}\right) 1\left(x_{i} / h_{n} \in A\left(\tilde{b}_{+, \infty}, \tilde{d}_{+, \infty}\right)\right) u_{i} \\
& +\frac{1}{\sqrt{n h}} \sum_{i=1}^{n}\left(\tilde{b}_{+, n}^{*}+\sum_{j=1}^{p-1} d_{+, n, j}^{*}\left(\frac{x_{i}}{h_{n}}\right)^{j}-C\left|\frac{x_{i}}{h_{n}}\right|^{p}\right) \\
& \cdot\left[1\left(x_{i} / h_{n} \in A\left(\tilde{b}_{+, n}^{*}, \tilde{d}_{+, n}^{*}\right)\right)-1\left(x_{i} / h_{n} \in A\left(\tilde{b}_{+, \infty}, \tilde{d}_{+, \infty}\right)\right)\right] u_{i} .
\end{aligned}
$$

The first term converges to zero uniformly in probability by Slutsky's Theorem. The second term can be written as a sum of terms of the form

$$
\frac{1}{\sqrt{n h_{n}}} \sum_{i=1}^{n}\left(x_{i} / h_{n}\right)^{j}\left[1\left(x_{i} / h_{n} \in A\left(\tilde{b}_{+, n}^{*}, \tilde{d}_{+, n}^{*}\right)\right)-1\left(x_{i} / h_{n} \in A\left(\tilde{b}_{+, \infty}, \tilde{d}_{+, \infty}\right)\right)\right] u_{i}
$$

times sequences that converge uniformly in probability to finite constants. To show that this 
converges in probability to zero uniformly over $\mathcal{F}, \mathcal{Q}_{n}$, note that, letting $u_{1}^{*}, \ldots, u_{k}^{*}$ be the positive zeros of the polynomial $\tilde{b}_{+, \infty}+\sum_{j=1}^{p-1} \tilde{d}_{+, j, \infty} u^{j}+C u^{p}$, the following statement will hold with probability approaching one uniformly over $\mathcal{F}, \mathcal{Q}_{n}$ for any $\eta>0$ : for all $u$ with $1\left(u \in A\left(\tilde{b}_{+, n}^{*}, \tilde{d}_{+, n}^{*}\right)\right)-1\left(u \in A\left(\tilde{b}_{+, \infty}, \tilde{d}_{+, \infty}\right)\right) \neq 0$, there exists $\ell$ such that $\left|u-u_{\ell}^{*}\right| \leq \eta$. It follows that the above display is, with probability approaching one uniformly over $\mathcal{F}, \mathcal{Q}_{n}$, bounded by a constant times the sum over $j=0, \ldots, p$ and $\ell=1, \ldots, k$ of

$$
\max _{-1 \leq t \leq 1}\left|\frac{1}{\sqrt{n h_{n}}} \sum_{i: u_{\ell}-\eta \leq x_{i} / h_{n} \leq u_{\ell}+t \eta}\left(x_{i} / h_{n}\right)^{j} u_{i}\right| .
$$

By Kolmogorov's inequality (see pp. 62-63 in Durrett, 1996), the probability of this quantity being greater than a given $\delta>0$ under a given $f, Q$ is bounded by

$$
\begin{aligned}
\frac{1}{\delta^{2}} \frac{1}{n h_{n}} \sum_{i: u_{\ell}-\eta \leq x_{i} / h_{n} \leq u_{\ell}+\eta} \operatorname{var}_{Q}\left[\left(x_{i} / h_{n}\right)^{j} u_{i}\right]=\frac{1}{\delta^{2}} \frac{1}{n h_{n}} \sum_{i: u_{\ell}-\eta \leq x_{i} / h_{n} \leq u_{\ell}+\eta}\left(x_{i} / h_{n}\right)^{2 j} \sigma^{2}\left(x_{i}\right) \\
\rightarrow \frac{p_{X,+}(0) \sigma_{+}^{2}(0)}{\delta^{2}} \int_{u_{\ell}^{*}-\eta}^{u_{\ell}^{*}+\eta} u^{2 j} d u,
\end{aligned}
$$

which can be made arbitrarily small by making $\eta$ small.

For the bias formulas, we have

$$
\begin{aligned}
\left|\overline{\operatorname{bias}}_{n}-\overline{\operatorname{bias}}_{n}^{*}\right|=\frac{C}{n h_{n}}\left|\sum_{i=1}^{n}\right| w_{n}\left(x_{i} / h_{n}\right) x_{i}^{p}\left|-\sum_{i=1}^{n}\right| w_{n}^{*}\left(x_{i} / h_{n}\right) x_{i}^{p}|| \\
\quad \leq \frac{C}{n h_{n}} \sum_{i=1}^{n}\left|w_{n}\left(x_{i} / h_{n}\right)-w_{n}^{*}\left(x_{i} / h_{n}\right)\right| \cdot\left|x_{i}\right|^{p} .
\end{aligned}
$$

This converges to zero when scaled by $n^{p /(2 p+1)}$ by arguments given above.

For the variance formulas, we have

$$
\begin{aligned}
&\left|\tilde{v}_{n}-\tilde{v}_{n}^{*}\right|=\frac{1}{\left(n h_{n}\right)^{2}}\left|\sum_{i=1}^{n} w_{n}\left(x_{i} / h_{n}\right)^{2} \tilde{\sigma}^{2}\left(x_{i}\right)-\sum_{i=1}^{n} w_{n}^{*}\left(x_{i} / h_{n}\right)^{2} \tilde{\sigma}^{* 2}\left(x_{i}\right)\right| \\
& \leq \frac{1}{\left(n h_{n}\right)^{2}} \sum_{i=1}^{n}\left|w_{n}\left(x_{i} / h_{n}\right)^{2} \tilde{\sigma}^{2}\left(x_{i}\right)-w_{n}^{*}\left(x_{i} / h_{n}\right)^{2} \tilde{\sigma}^{* 2}\left(x_{i}\right)\right| \\
& \quad \leq \frac{1}{n h_{n}} \max _{|x| \leq B}\left|w_{n}(x)^{2} \tilde{\sigma}^{2}(x)-w_{n}^{*}(x)^{2} \tilde{\sigma}^{* 2}(x)\right| \cdot \frac{1}{n h_{n}} \sum_{i=1}^{n} 1\left(\left|x_{i} / h_{n}\right| \leq B\right)
\end{aligned}
$$


with probability approaching one where $B$ is a bound on the support of $w_{n}(x)$ and $w_{n}^{*}(x)$ that holds with probability approaching one. Since $\frac{1}{n h_{n}} \sum_{i=1}^{n} 1\left(\left|x_{i} / h_{n}\right| \leq B\right)$ converges to a constant by Assumption H.1 and $\tilde{v}_{n}=n^{-2 p /(2 p+1)} v_{\infty}(1+o(1))=\left(n h_{n}\right)^{-1} v_{\infty}(1+o(1))$, dividing the above display by $\tilde{v}_{n}$ gives an expression that is bounded by a constant times $\max _{|x| \leq B h_{n}}\left|w_{n}(x)^{2} \tilde{\sigma}^{2}(x)-w_{n}^{*}(x)^{2} \tilde{\sigma}^{* 2}(x)\right|$, which converges uniformly in probability to zero.

We are now ready to prove Theorem H.3. First, consider the case with $\tilde{\sigma}(\cdot)$ is deterministic and Assumption H.4 holding. By Lemma H.7, $\delta_{n} \rightarrow \delta_{\infty}$. By Lemma H.6, it then follows that, under Assumption H.4, $n^{p /(2 p+1)} w_{n}\left(\delta_{n}\right) \rightarrow \omega_{\infty}\left(\delta_{\infty}\right)$ so that Lemma H.8 applies to show that Assumption H.5 holds with $k^{+}(x)=k_{\tilde{\sigma}_{+}(0)}^{+}\left(x ; \tilde{b}_{+, \infty}, \tilde{d}_{+, \infty}\right)$ and $k^{-}(x)=k_{\tilde{\sigma}_{-}(0)}^{-}\left(x ; \tilde{b}_{-, \infty}, \tilde{d}_{-, \infty}\right)$, where $\left(\tilde{b}_{+, \infty}, \tilde{d}_{+, \infty}, \tilde{b}_{-, \infty}, \tilde{d}_{-, \infty}\right)$ minimize $G_{\infty}\left(\tilde{b}_{+, \infty}, \tilde{d}_{+, \infty}, \tilde{b}_{-, \infty}, \tilde{d}_{-, \infty}\right)$ subject to $\tilde{b}_{+, \infty}+\tilde{b}_{-, \infty}=$ $\omega_{\infty}\left(\delta_{\infty}\right) / 2$. The coverage statements and convergence of $n^{p /(2 p+1)} \hat{\chi}$ then follow from Theorem H.1 and by calculating $\overline{\operatorname{bias}}_{\infty}$ and $v_{\infty}$ in terms of the limiting modulus.

We now prove the optimality statements (under which the assumption was made that, for each $n$, there exists a $Q \in \mathcal{Q}_{n}$ such that the errors are normally distributed). In this case, for any $\eta>0$, if a linear estimator $\tilde{L}$ and constant $\chi$ satisfy

$$
\inf _{f \in \mathcal{F}, Q \in \mathcal{Q}_{n}} P\left(L f \in\left\{\tilde{L} \pm n^{-p /(2 p+1)} \chi\right\}\right) \geq 1-\alpha-\eta
$$

we must have $\chi \geq \sup _{\delta>0} \frac{n^{p /(2 p+1)} \omega_{\sigma(\cdot), n}(\delta)}{\delta} \chi_{A, \alpha+\eta}(\delta / 2)$ by the results of Donoho (1994) (using the characterization of optimal half-length at the beginning of Supplemental Appendix $\mathrm{G}$ ). This converges to $\sup _{\delta>0} \frac{\omega_{\infty}(\delta)}{\delta} \chi_{A, \alpha+\eta}(\delta / 2)$ by Lemma H.7. If $\liminf _{n} \inf _{f \in \mathcal{F}, Q \in \mathcal{Q}_{n}} P(L f \in$ $\left.\left\{\tilde{L} \pm n^{-p /(2 p+1)} \chi\right\}\right) \geq 1-\alpha$, then, for any $\eta>0$, the above display must hold for large enough $n$, so that $\chi \geq \lim _{\eta \downarrow 0} \sup _{\delta>0} \frac{\omega_{\infty}(\delta)}{\delta} \chi_{A, \alpha+\eta}(\delta / 2)=\sup _{\delta>0} \frac{\omega_{\infty}(\delta)}{\delta} \chi_{A, \alpha}(\delta / 2)$ (the limit with respect to $\eta$ follows since there exist $0<\underline{\delta}<\bar{\delta}<\infty$ such that the supremum over $\delta$ is taken on $[\underline{\delta}, \bar{\delta}]$ for $\eta$ in a neighborhood of zero, and since $\chi_{A, \alpha}(\delta / 2)$ is continuous with respect to $\alpha$ uniformly over $\delta$ in compact sets).

For the asymptotic efficiency bound regarding expected length among all confidence intervals, note that, for any $\eta>0$, any CI satisfying the asymptotic coverage requirement must be a $1-\alpha-\eta$ CI for large enough $n$, which means that, since the CI is valid under the $Q_{n} \in \mathcal{Q}_{n}$ where the errors are normal, the expected length of the CI at $f=0$ and this $Q_{n}$ scaled by $n^{p /(2 p+1)}$ is at least

$$
(1-\alpha-\eta) E\left[n^{p /(2 p+1)} \omega_{\sigma(\cdot), n}\left(2\left(z_{1-\alpha-\eta}-Z\right)\right) \mid Z \leq z_{1-\alpha-\eta}\right]
$$


by Corollary 3.3. This converges to $(1-\alpha-\eta) E\left[\omega_{\infty}\left(2\left(z_{1-\alpha-\eta}-Z\right)\right) \mid Z \leq z_{1-\alpha-\eta}\right]$ by Lemma H.7. The result follows from taking $\eta \rightarrow 0$ and using the dominated convergence theorem, and using the fact that $\omega_{\infty}(\delta)=\omega_{\infty}(1) \delta^{2 p /(2 p+1)}$. The asymptotic efficiency bounds for the feasible one-sided CI follow from similar arguments, using Theorem 3.1 and Corollary 3.2 along with Theorem H.1 and Lemma G.3.

In the case where Assumption H.3 holds rather than Assumption H.4, it follows from Lemma H.7 that $\delta_{n} \underset{\mathcal{F}, \mathcal{Q}_{n}}{\stackrel{p}{\rightarrow}} \delta_{\infty}$. Then, by Lemma H.9, the conditions in the last display of Theorem H.1 hold with $\hat{L}_{\delta_{n}, \tilde{\sigma}(\cdot)}$ playing the role of $\hat{L}^{*}$ and $\hat{L}_{\delta_{n}, \sigma(\cdot)}$ playing the role of $\hat{L}$. The results then follow from Theorem H.1 and the arguments above applied to the CIs based on $\hat{L}_{\delta_{n}, \sigma(\cdot)} \cdot$

\section{References}

Armstrong, T. B. And M. Kolesár (2016a): "Optimal inference in a class of regression models," ArXiv:1511.06028v2.

- (2016b): "Simple and honest confidence intervals in nonparametric regression," ArXiv: 1606.01200.

Brown, L. D. AND M. G. Low (1996): "Asymptotic equivalence of nonparametric regression and white noise," Annals of Statistics, 24, 2384-2398.

Brown, L. D. And C.-H. Zhang (1998): “Asymptotic Nonequivalence of Nonparametric Experiments When the Smoothness Index is 1/2," The Annals of Statistics, 26, 279-287.

Calonico, S., M. D. Cattaneo, and R. Titiunik (2014): "Robust Nonparametric Confidence Intervals for Regression-Discontinuity Designs," Econometrica, 82, 2295-2326.

Cheng, M.-Y., J. FAn, And J. S. Marron (1997): "On automatic boundary corrections," The Annals of Statistics, 25, 1691-1708.

Chernozhukov, V., S. Lee, And A. M. Rosen (2013): "Intersection Bounds: Estimation and Inference," Econometrica, 81, 667-737.

Donoho, D. L. (1994): "Statistical Estimation and Optimal Recovery," The Annals of Statistics, 22, 238-270. 
Donoho, D. L. And M. G. Low (1992): "Renormalization Exponents and Optimal Pointwise Rates of Convergence," The Annals of Statistics, 20, 944-970.

Drees, H. (1999): "On fixed-length confidence intervals for a bounded normal mean," Statistics \&3 Probability Letters, 44, 399-404.

Durrett, R. (1996): Probability: Theory and Examples, Belmont, California: Duxbury Press, 2nd ed.

FAn, J. (1993): "Local Linear Regression Smoothers and Their Minimax Efficiencies," The Annals of Statistics, 21, 196-216.

Imbens, G. W. and K. Kalyanaraman (2012): "Optimal bandwidth choice for the regression discontinuity estimator," The Review of Economic Studies, 79, 933-959.

LEe, D. S. (2008): "Randomized experiments from non-random selection in U.S. House elections," Journal of Econometrics, 142, 675-697.

Nussbaum, M. (1996): "Asymptotic equivalence of density estimation and Gaussian white noise," The Annals of Statistics, 24, 2399-2430.

ReIss, M. (2008): "Asymptotic Equivalence for Nonparametric Regression with Multivariate and Random Design," Annals of Statistics, 36, 1957-1982.

Sacks, J. AND D. Ylvisaker (1978): "Linear Estimation for Approximately Linear Models," The Annals of Statistics, 6, 1122-1137.

Stock, J. And M. Yogo (2005): "Testing for Weak Instruments in Linear IV Regression," in Identifcation and Inference for Econometric Models: Essays in Honor of Thomas Rothenberg, ed. by D. W. K. Andrews and J. Stock, Cambgridge, UK: Cambridge University Press, 80-108.

van der Vaart, A. W. (1998): Asymptotic Statistics, Cambridge, UK ; New York, NY, USA: Cambridge University Press.

von Bahr, B. And C.-G. Esseen (1965): "Inequalities for the $r$ th Absolute Moment of a Sum of Random Variables, $1 \leq r \leq 2$," The Annals of Mathematical Statistics, 36, 299-303. 


\begin{tabular}{|c|c|c|c|c|c|c|}
\hline \multirow[b]{2}{*}{ CI method } & \multicolumn{3}{|c|}{$\sigma^{2}=0.1295$} & \multicolumn{3}{|c|}{$\sigma^{2}=4 \cdot 0.1295$} \\
\hline & Cov. $(\%)$ & Bias & RL & Cov. $(\%)$ & Bias & RL \\
\hline \multicolumn{7}{|c|}{ Design $1,\left(b_{1}, b_{2}\right)=(0.45,0.75)$} \\
\hline Conventional, $\hat{h}_{I K}$ & 10.1 & -0.098 & 0.54 & 81.7 & -0.099 & 0.72 \\
\hline $\mathrm{RBC}, \hat{h}_{I K}, \rho=1$ & 64.4 & -0.049 & 0.80 & 93.9 & -0.050 & 1.06 \\
\hline Conventional, $\hat{h}_{C C T}$ & 91.2 & -0.010 & 1.01 & 92.7 & -0.010 & 1.26 \\
\hline $\mathrm{RBC}, \hat{h}_{C C T}$ & 93.7 & 0.003 & 1.18 & 93.6 & 0.007 & 1.48 \\
\hline FLCI, $C=1$ & 94.6 & -0.024 & 1 & 94.9 & -0.069 & 1 \\
\hline FLCI, $C=3$ & 96.7 & -0.009 & 1.25 & 96.5 & -0.028 & 1.25 \\
\hline \multicolumn{7}{|c|}{ Design $2,\left(b_{1}, b_{2}\right)=(0.4,0.9)$} \\
\hline Conventional, $\hat{h}_{I K}$ & 54.2 & -0.063 & 0.68 & 89.6 & -0.085 & 0.77 \\
\hline $\mathrm{RBC}, \hat{h}_{I K}, \rho=1$ & 94.8 & -0.006 & 1.00 & 95.9 & -0.043 & 1.13 \\
\hline Conventional, $\hat{h}_{C C T}$ & 91.4 & -0.009 & 1.02 & 92.7 & -0.009 & 1.26 \\
\hline $\mathrm{RBC}, \hat{h}_{C C T}$ & 93.6 & 0.003 & 1.19 & 93.6 & 0.007 & 1.49 \\
\hline FLCI, $C=1$ & 94.5 & -0.024 & 1 & 95.0 & -0.065 & 1 \\
\hline FLCI, $C=3$ & 96.8 & -0.009 & 1.25 & 96.5 & -0.028 & 1.25 \\
\hline \multicolumn{7}{|c|}{ Design $3,\left(b_{1}, b_{2}\right)=(0.25,0.65)$} \\
\hline Conventional, $\hat{h}_{I K}$ & 87.8 & -0.030 & 0.74 & 91.4 & -0.009 & 0.76 \\
\hline $\mathrm{RBC}, \hat{h}_{I K}, \rho=1$ & 94.8 & -0.014 & 1.09 & 95.0 & -0.044 & 1.12 \\
\hline Conventional, $\hat{h}_{C C T}$ & 90.9 & -0.014 & 0.97 & 92.8 & -0.013 & 1.25 \\
\hline $\mathrm{RBC}, \hat{h}_{C C T}$ & 92.2 & -0.009 & 1.14 & 93.5 & -0.007 & 1.48 \\
\hline FLCI, $C=1$ & 94.7 & -0.022 & 1 & 96.7 & -0.028 & 1 \\
\hline FLCI, $C=3$ & 96.8 & -0.009 & 1.25 & 96.6 & -0.025 & 1.25 \\
\hline \multicolumn{7}{|l|}{ Design $4, f(x)=0$} \\
\hline Conventional, $\hat{h}_{I K}$ & 93.2 & 0.000 & 0.54 & 93.2 & -0.001 & 0.72 \\
\hline $\mathrm{RBC}, \hat{h}_{I K}, \rho=1$ & 95.2 & 0.000 & 0.80 & 95.2 & 0.001 & 1.06 \\
\hline Conventional, $\hat{h}_{C C T}$ & 93.1 & 0.001 & 0.94 & 93.1 & 0.003 & 1.25 \\
\hline $\mathrm{RBC}, \hat{h}_{C C T}$ & 93.5 & 0.001 & 1.12 & 93.5 & 0.004 & 1.48 \\
\hline FLCI, $C=1$ & 96.8 & 0.001 & 1 & 96.9 & 0.000 & 1 \\
\hline FLCI, $C=3$ & 96.8 & 0.001 & 1.25 & 96.8 & 0.002 & 1.25 \\
\hline
\end{tabular}

Table S1: Monte Carlo simulation, $C=1$. Coverage ("Cov") and relative length relative to optimal fixed-length CI for $\mathcal{F}_{R D H, 2}(1)$ ("RL"). "Bias" refers to bias of estimator around which CI is centered. 11,000 simulation draws. 


\begin{tabular}{|c|c|c|c|c|c|c|}
\hline \multirow[b]{2}{*}{ CI method } & \multicolumn{3}{|c|}{$\sigma^{2}=0.1295$} & \multicolumn{3}{|c|}{$\sigma^{2}=4 \cdot 0.1295$} \\
\hline & Cov. $(\%)$ & Bias & RL & Cov. $(\%)$ & Bias & RL \\
\hline \multicolumn{7}{|c|}{ Design $1,\left(b_{1}, b_{2}\right)=(0.45,0.75)$} \\
\hline Conventional, $\hat{h}_{I K}$ & 0.1 & -0.292 & 0.44 & 22.4 & -0.296 & 0.58 \\
\hline $\mathrm{RBC}, \hat{h}_{I K}, \rho=1$ & 27.1 & -0.127 & 0.65 & 77.8 & -0.149 & 0.85 \\
\hline Conventional, $\hat{h}_{C C T}$ & 89.3 & -0.019 & 0.94 & 91.6 & -0.031 & 1.05 \\
\hline $\mathrm{RBC}, \hat{h}_{C C T}$ & 93.7 & 0.004 & 1.06 & 93.7 & 0.012 & 1.22 \\
\hline FLCI, $C=1$ & 67.3 & -8.078 & 0.80 & 73.1 & -0.209 & 0.80 \\
\hline FLCI, $C=3$ & 94.5 & -0.032 & 1 & 94.6 & -0.089 & 1 \\
\hline \multicolumn{7}{|c|}{ Design $2,\left(b_{1}, b_{2}\right)=(0.4,0.9)$} \\
\hline Conventional, $\hat{h}_{I K}$ & 60.0 & -0.071 & 0.71 & 71.4 & -0.193 & 0.72 \\
\hline $\mathrm{RBC}, \hat{h}_{I K}, \rho=1$ & 93.5 & 0.000 & 1.04 & 95.1 & -0.020 & 1.05 \\
\hline Conventional, $\hat{h}_{C C T}$ & 89.7 & -0.018 & 0.95 & 91.7 & -0.029 & 1.05 \\
\hline $\mathrm{RBC}, \hat{h}_{C C T}$ & 93.6 & 0.004 & 1.09 & 93.6 & 0.012 & 1.24 \\
\hline FLCI, $C=1$ & 70.3 & -0.073 & 0.80 & 76.3 & -0.197 & 0.80 \\
\hline FLCI, $C=3$ & 94.3 & -0.030 & 1 & 94.6 & -0.089 & 1 \\
\hline \multicolumn{7}{|c|}{ Design $3,\left(b_{1}, b_{2}\right)=(0.25,0.65)$} \\
\hline Conventional, $\hat{h}_{I K}$ & 79.9 & -0.052 & 0.76 & 89.2 & -0.085 & 0.73 \\
\hline $\mathrm{RBC}, \hat{h}_{I K}, \rho=1$ & 93.3 & 0.001 & 1.13 & 94.6 & -0.072 & 1.07 \\
\hline Conventional, $\hat{h}_{C C T}$ & 80.7 & -0.032 & 0.87 & 91.8 & -0.042 & 1.01 \\
\hline $\mathrm{RBC}, \hat{h}_{C C T}$ & 86.2 & -0.017 & 1.00 & 92.7 & -0.027 & 1.20 \\
\hline FLCI, $C=1$ & 73.5 & -0.069 & 0.8 & 93.8 & -0.084 & 0.80 \\
\hline FLCI, $C=3$ & 94.4 & -0.030 & 1 & 95.1 & -0.078 & 1 \\
\hline \multicolumn{7}{|l|}{ Design $5, f(x)=0$} \\
\hline Conventional, $\hat{h}_{I K}$ & 93.2 & 0.000 & 0.43 & 93.2 & -0.001 & 0.57 \\
\hline $\mathrm{RBC}, \hat{h}_{I K}, \rho=1$ & 95.2 & 0.000 & 0.64 & 95.2 & 0.001 & 0.85 \\
\hline Conventional, $\hat{h}_{C C T}$ & 93.1 & 0.001 & 0.75 & 93.1 & 0.003 & 1.00 \\
\hline $\mathrm{RBC}, \hat{h}_{C C T}$ & 93.5 & 0.001 & 0.89 & 93.5 & 0.004 & 1.18 \\
\hline FLCI, $C=1$ & 96.8 & 0.001 & 0.80 & 96.9 & 0.000 & 0.80 \\
\hline FLCI, $C=3$ & 96.8 & 0.001 & 1 & 96.7 & 0.002 & 1 \\
\hline
\end{tabular}

Table S2: Monte Carlo simulation, $C=3$. Coverage ("Cov") and relative length relative to optimal fixed-length CI for $\mathcal{F}_{R D H, 2}(1)$ ("RL"). "Bias" refers to bias of estimator around which CI is centered. 11,000 simulation draws. 


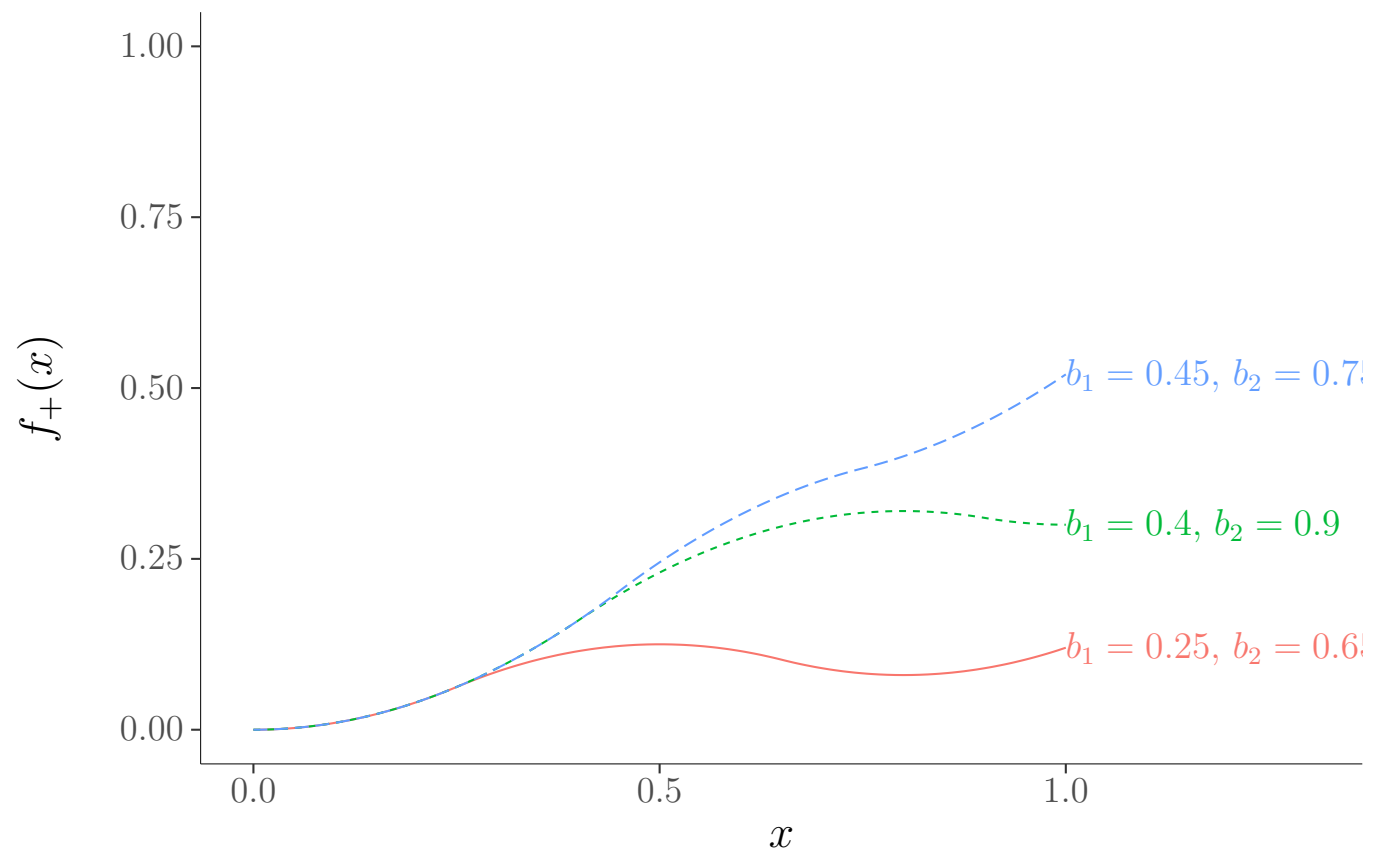

Figure S1: Regression function for Monte Carlo simulation, Designs 1-3, and $C=1$. Knots $b_{1}=0.45, b_{2}=0.75$ correspond to Design $1, b_{1}=0.4, b_{2}=0.9$ to Design 2 , and $b_{1}=$ $0.25, b_{2}=0.65$ to Design 3 . 